\title{
Characterization of neuronal SNAREs and interacting proteins
}

\author{
PhD thesis
}

in partial fulfillment of the requirements

for the degree "Doctor of Philosophy (PhD)"

in the molecular biology program

at the Georg August University Göttingen

Faculty of Biology

\section{Submitted by}

Ajaybabu Pobbati Venkatesan

born in

Chennai, India 
I hereby declare that the Ph.D. thesis entitled "Characterization of neuronal SNAREs and interacting proteins" has been written independently and with no other sources and aids than quoted. 


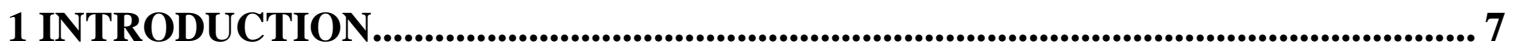

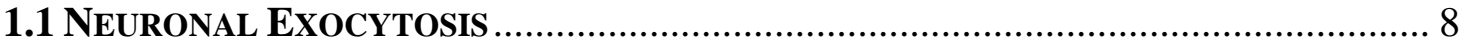

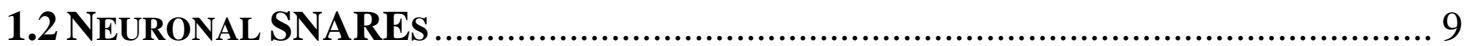

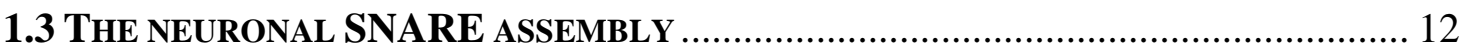

1.3.1 Intermediate step in neuronal SNARE assembly ................................ 15

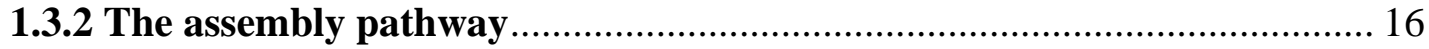

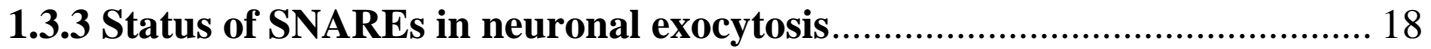

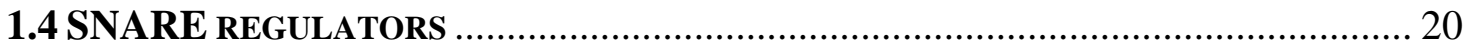

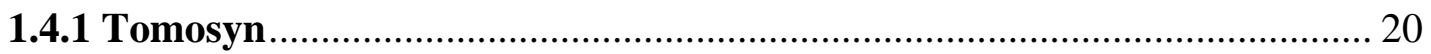

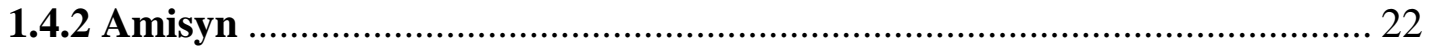

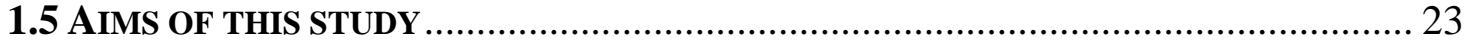

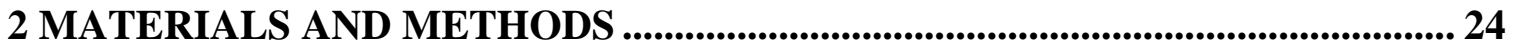

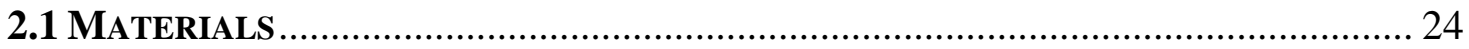

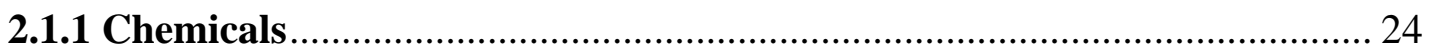

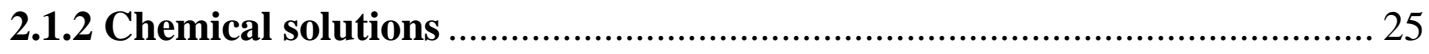

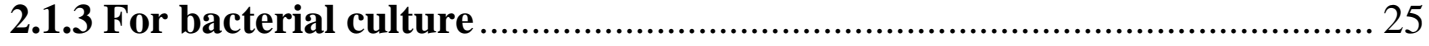

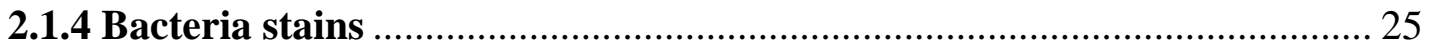

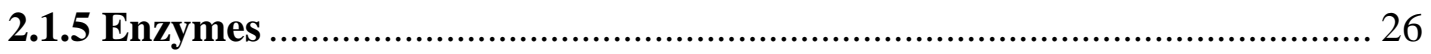

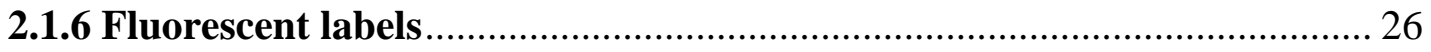

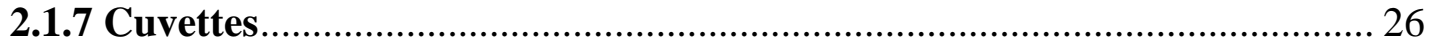

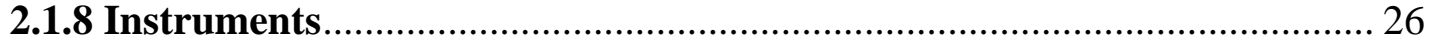

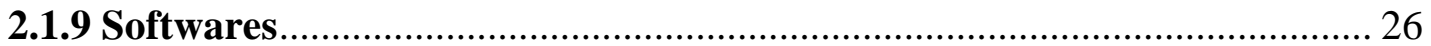

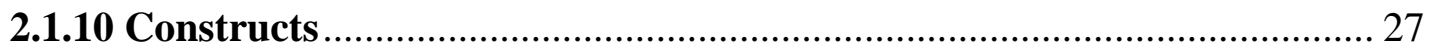

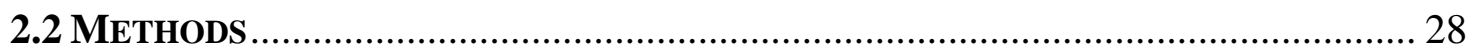

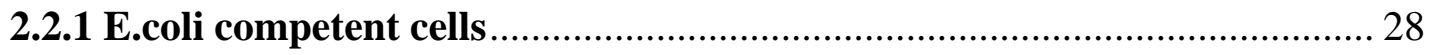

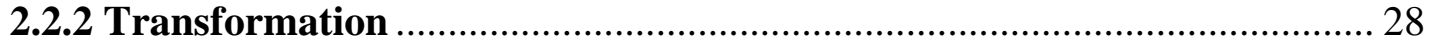

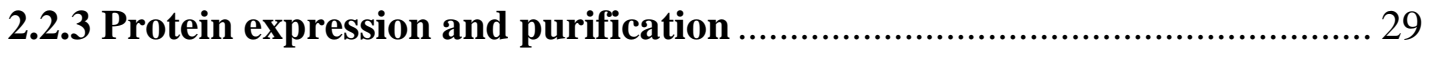

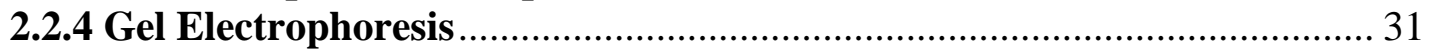

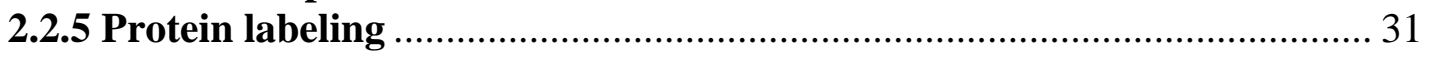

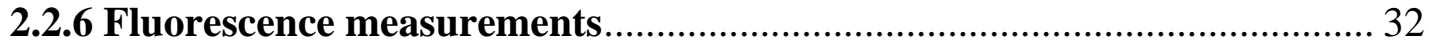

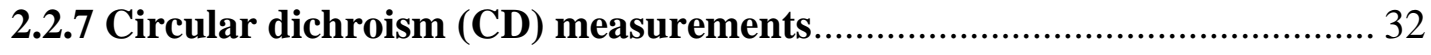

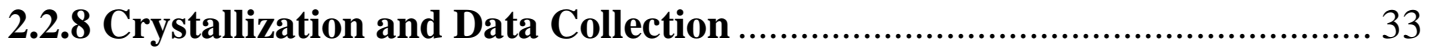

2.2.9 Structure Determination and Refinement ........................................... 33

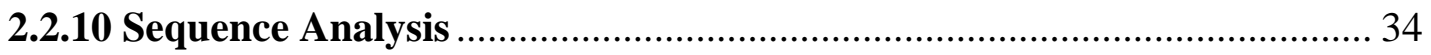

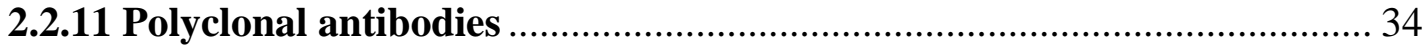

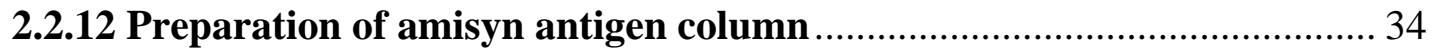

2.2.13 Affinity purification of Amisyn antibodies ....................................... 35

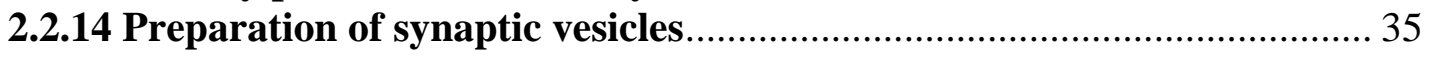

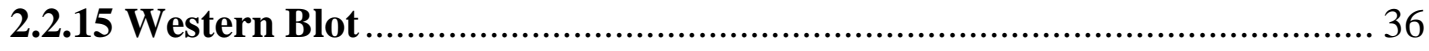

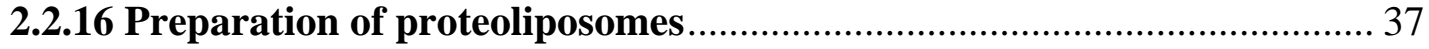

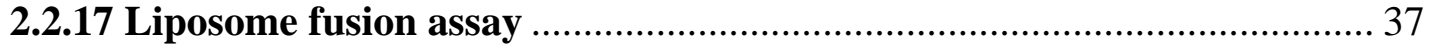


3 RESULTS ..................................................................................................................... 38

3.1 A HELIX REPLACEMENT REACTION SHEDS NEW LIGHT ON SNARE MEDIATED

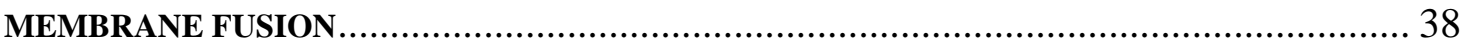

3.1.1 Replacement of the synaptobrevin helix ............................................ 38

3.1.2 Fast binding of synaptobrevin to $\Delta \mathbf{N}$ ternary complexes ........................ 40

3.1.3 Shortened synaptobrevin replacement kinetics ................................... 42

3.1.4 Shortening synaptobrevin enhances the replacement rate ...................... 43

3.1.5 An open binding site is required for rapid binding ................................ 45

3.1.6 $\alpha$-helical structure in the $\mathbf{N}$-terminal half of the $\Delta \mathbf{N}$ ternary complex..... 46

3.1.7 A SNARE complex containing two synaptobrevin halves unfolds in two

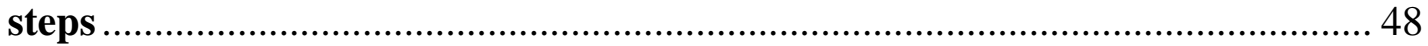

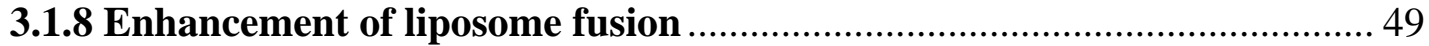

3.2 STRUCTURAL BASIS FOR THE INHIBITORY ROLE OF TOMOSYN IN EXOCYTOSIS ... 53

3.2.1 Structure of the Tomosyn SNARE complex .......................................... 53

3.2.2 The tomosyn SNARE complex is a dead end complex ........................... 55

3.2.3 Tomosyn and synaptobrevin SNARE complexes assemble at similar rates

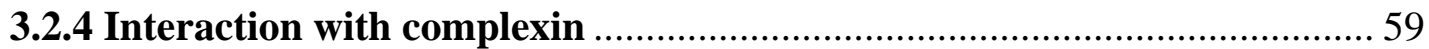

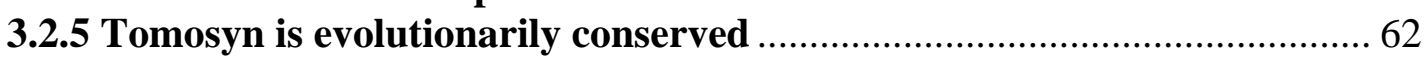

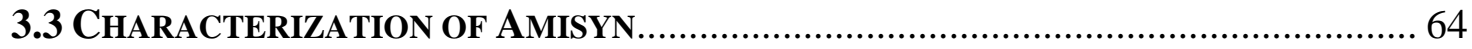

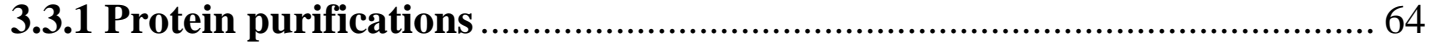

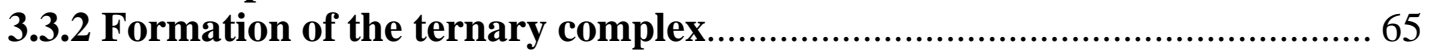

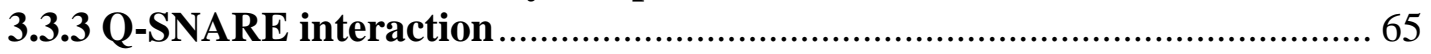

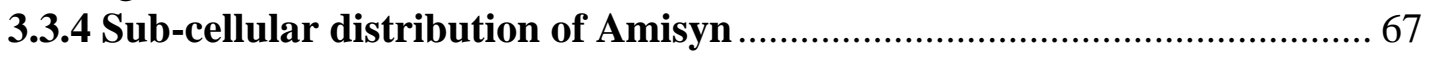

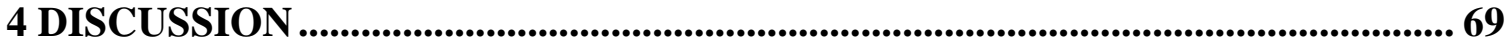

4.1 HELIX REPLACEMENT MECHANISM SHEDS NEW LIGHT ON SNARE MEDIATED

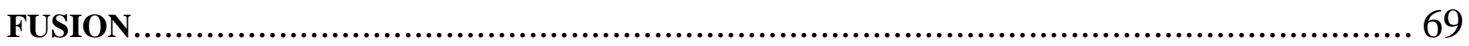

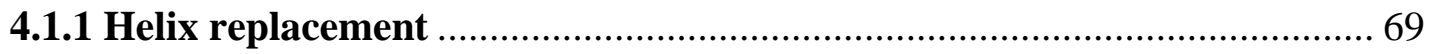

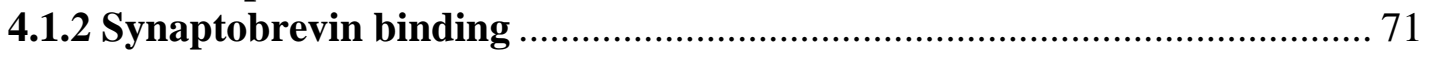

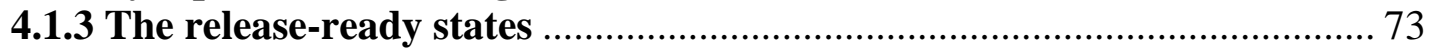

4.2 STRUCTURAL BASIS FOR THE INHIBITORY ROLE OF TOMOSYN IN EXOCYTOSIS ... 77

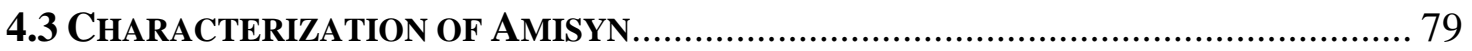

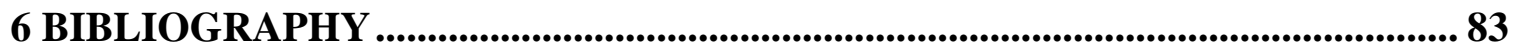

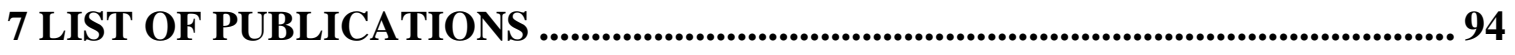

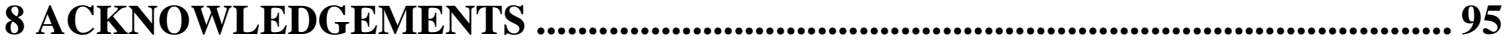

9 APPENDIX ......................................................................................................................... 96

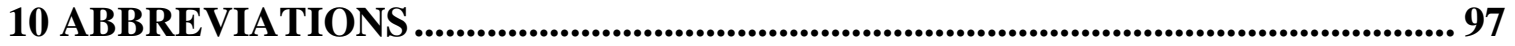

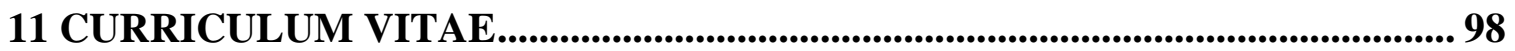




\section{Table of figures}

Figure 1 N-terminal domains of SNAREs ................................................................ 8

Figure 2 Domain structure of neuronal SNAREs ....................................................... 10

Figure 3 Structure of the neuronal SNARE complex ……………………………...... 12

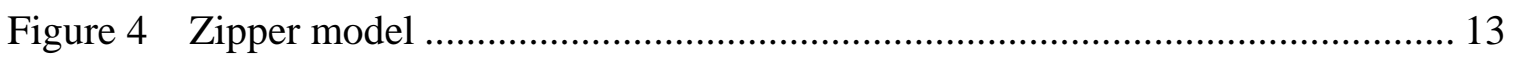

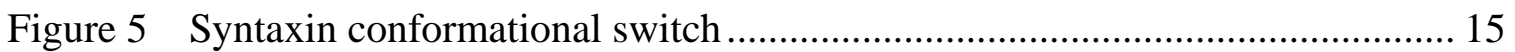

Figure 6 Current model of SNARE assembly pathway ................................................ 17

Figure 7 Partially and fully assembled SNAREs........................................................... 20

Figure 8 Alignment of SNARE motifs .................................................................... 39

Figure 9 Replacement of the synaptobrevin helix ......................................................... 40

Figure 10 Fast binding of synaptobrevin to $\Delta \mathrm{N}$ ternary complexes ............................... 41

Figure 11 Shortened synaptobrevin replacement kinetics ............................................... 43

Figure 12 Displacement of Sb (49-96) monitored by fluorescence anisotropy .............. 45

Figure 13 An open-binding site is required for rapid binding ....................................... 46

Figure $14 \alpha$ - helical structure in the $\mathrm{N}$-terminal half of the $\Delta \mathrm{N}$ ternary complex........... 47

Figure 15 A SNARE complex containing the two synaptobrevin halves unfolds in two

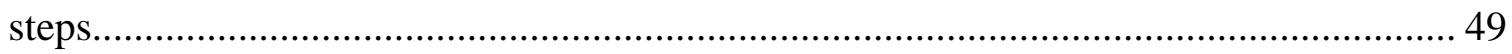

Figure 16 The $\Delta \mathrm{N}$ complex accelerates liposome fusion................................................ 51

Figure 17 A model of the faster fusion pathway offered by the $\Delta$ N ternary complex.... 52

Figure 18 Structure of the Tomosyn SNARE complex ................................................... 55

Figure 19 The tomosyn SNARE complex is a dead end complex................................... 56

Figure 20 The R-SNARE helix cannot be replaced ........................................................ 57

Figure 21 The synaptobrevin and the tomosyn SNARE complex form with similar rates

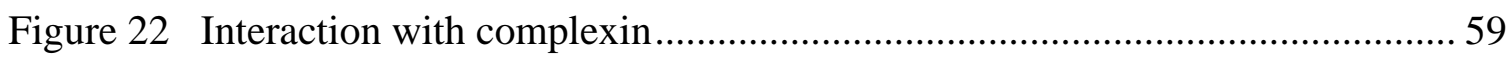

Figure 23 Complexin does not bind to the tomosyn SNARE complex ...........................6 60

Figure 24 Evaluation of the interaction between complexin and the SNARE complex

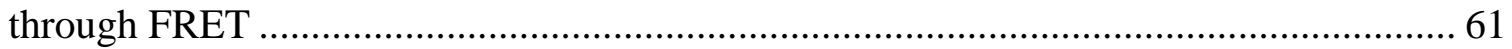

Figure 25 Tomosyn is evolutionarily conserved.......................................................... 63

Figure 26 Purification of full-length and SNARE motif of amisyn................................. 64 


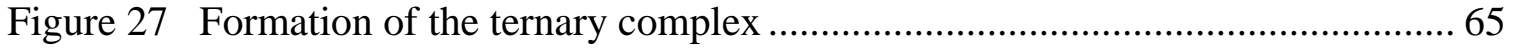

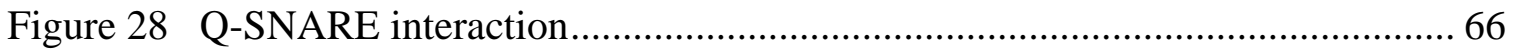

Figure 29 Structural changes after the formation of amisyn SNARE complex.............. 67

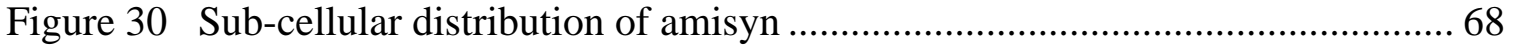

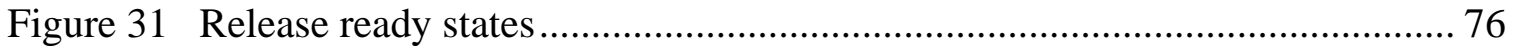




\section{Introduction}

The eukaryotic cell contains several membrane enclosed compartments (organelles) and each carries out a specialized function. Homeostasis requires selective exchange of material between the compartments as well as in and out of the cell. One mechanism by which eukaryotes accomplish selective exchange is by means of vesicular transport. Vesicles bud from a source compartment and fuse with the target compartment. Selection occurs during vesicle budding and fusion. Vesicle budding is mediated by coat proteins, whereas a superfamily of proteins, namely the SNAREs (soluble Nethylmaleimide-sensitive factor attachment protein receptors) mediates the fusion events (for reviews [1-6]).

All the vesicular fusion steps in the secretory, lysosomal/vacuolar and endocytotic pathways occur primarily through SNAREs. For example, secretory proteins are incorporated into vesicles that bud from the endoplasmic reticulum (ER) and through vesicular budding and SNARE mediated fusion they are transported to cis-, medial- and trans Golgi, before they finally fuse with the plasma membrane. Although most of the intracellular fusion events are mediated by SNAREs, some do not involve SNAREs, notably the homotypic fusion between mitochondria [7, 8].

The characteristic feature of SNARE proteins is the presence of a SNARE motif, a $\sim 60$ amino acid region of heptad repeats that forms coiled coils. The SNAREs are usually C-terminally anchored, either by means of a transmembrane domain or with the help of lipid anchors. The SNARE motif is normally adjacent to the membrane anchor and faces the cytoplasm. The extreme N-terminal domains of SNAREs are highly variable and structures of few $\mathrm{N}$-terminal domains are known (Fig. 1). These include (a) three helical bundle with a left handed twist, as seen in syntaxin 1a, syntaxin 6 and Vam 3p [9-12] (b) longin fold that contains five central $\beta$ sheets sandwiched by two $\alpha$-helices on one face and one $\alpha$-helix on the other face $[13,14]$ (c) PX domain that binds PtdIns(3)P [15]. 

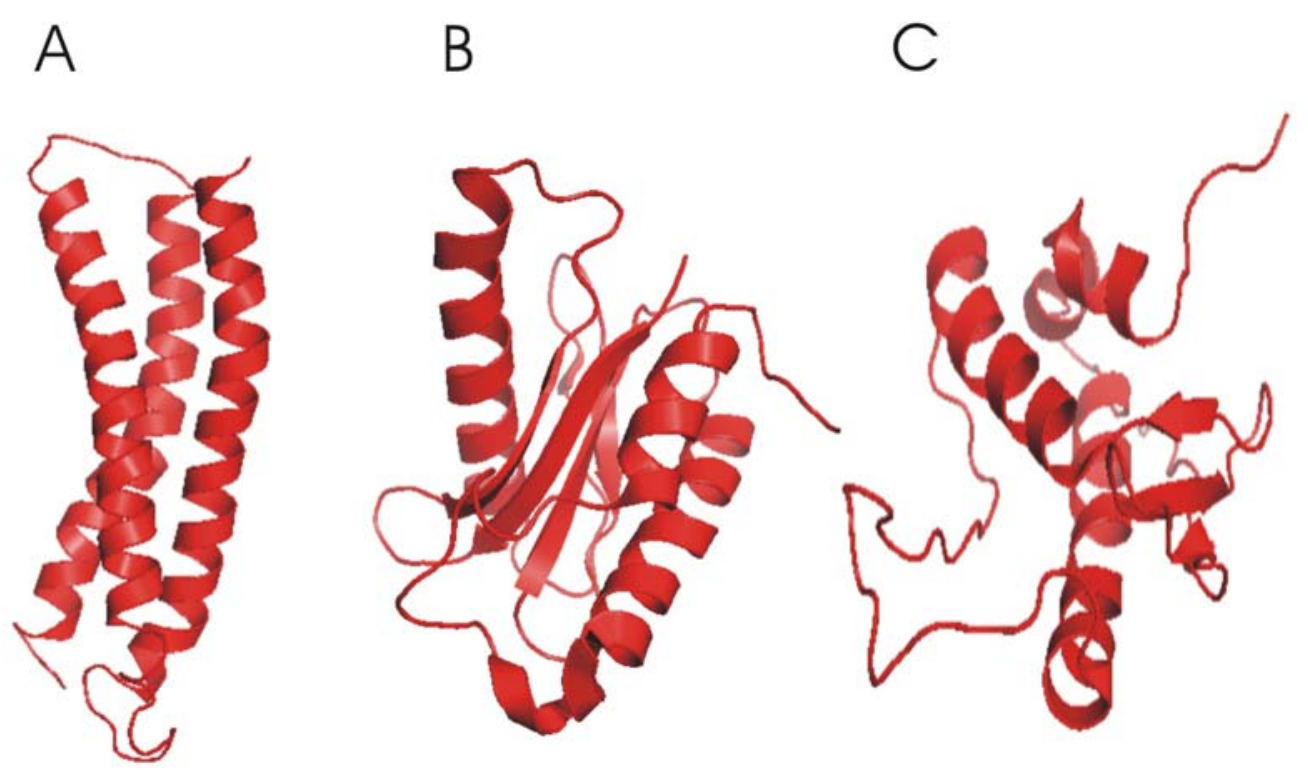

Figure 1 N-terminal domains of SNAREs

Structures of few N-terminal domains of SNAREs are known (A). N-terminal domain (also called $\mathrm{H}_{\mathrm{abc}}$ domain) of syntaxin 1a showing an anti-parallel triple helix with a left handed twist [10] (B). $\mathrm{N}$-terminal domain of Ykt6, a longin fold that contains five central $\beta$ sheets sandwiched by two helices on one face and one helix on the other face [14] (C) PX domain of Vam7p, which binds to PtdIns(3)P [15].

The SNAREs were originally classified as target and vesicle SNAREs ( $\mathrm{t}$ - and vSNAREs). This classification was based on the preferential localization of SNAREs, which is either on the target membrane or on the vesicle [16, 17]. SNAREs are proclaimed as fusion machines, it has been suggested that the SNARE assembly bridges vesicle and target membrane and perhaps releases sufficient energy that is needed to cause fusion.

\subsection{Neuronal Exocytosis}

Exocytosis is a membrane fusion event where vesicles fuse with the plasma membrane of the cell. It is used to restore the plasma membrane, discharge vesicular content (secretion) or to translocate membrane receptors. Exocytosis can be either constitutive or regulated. In constitutive exocytosis the vesicles continuously fuse with 
the plasma membrane, whereas in regulated exocytosis the fusion occurs after the arrival of an external signal.

Three modes of exocytosis occur in neurons (1) synchronous release or fast exocytosis (2) asynchronous release or delayed exocytosis and (3) spontaneous release or 'minis'. Synchronous release occurs due to action potential triggered local $\mathrm{Ca}^{2+}$ influx and several vesicles synchronously fuse in a millisecond timescale. Asynchronous release occurs due to a bulk increase of $\mathrm{Ca}^{2+}$ and it is relatively slower than the synchronous release. Spontaneous releases on the other hand are random fusion events, which are not triggered by $\mathrm{Ca}^{2+}$. Therefore both synchronous and asynchronous releases are regulated forms of exocytosis and the external signal in these cases is calcium.

Neurons establish communication through exocytosis of synaptic vesicles that are packed with chemical messengers. The communication point is referred to as synapse, the neuron that sends the signal through exocytosis is called the presynaptic neuron and the neuron that receives the signal is called the postsynaptic neuron. The exocytosis occurs at a specialized site, which is referred to as the active zone. As in all membrane fusion events, SNAREs play an important role in neuronal exocytosis. Speed and high regulation are characteristic of neuronal exocytosis. To achieve high speed and regulation, as in the case of fast exocytosis, the fusion machinery (namely the SNAREs) should be kept at a specialized state or perhaps the final steps of fusion is mediated by other factors. This is an intensively researched area and the research on neuronal SNAREs and its interacting factors continue to shed light on the molecular mechanism of neuronal exocytosis.

\subsection{Neuronal SNAREs}

Syntaxin 1, SNAP-25, and synaptobrevin (VAMP) are the SNAREs that are involved in neuronal exocytosis. Syntaxin 1 [18] is predominantly localized at the neuronal plasma membrane. Syntaxin 1 has two isoforms, which are called syntaxin 1a and syntaxin $1 \mathrm{~b}$ and are $84 \%$ identical in their amino acid sequence and are differentially distributed in the central and peripheral nervous system [19, 20]. Syntaxin1 is a type II 
membrane protein and is anchored to the membrane with the help of a single transmembrane domain. Syntaxin 1 contains a SNARE motif and a $\mathrm{H}_{\mathrm{abc}}$ domain (Fig. 2) (three helical bundle with a left handed twist, see Fig. 1). SNAP-25 [21] contains two SNARE motifs with a flexible linker between them. Like syntaxin, it is also primarily localized at the neuronal plasma membrane. However SNAP-25 does not contain a transmembrane anchor and is attached to the membrane with the help of palmitoyl modifications [22] (Fig. 2). SNAP-25 has two splice variants, SNAP-25A and SNAP25B, which differ at only nine amino acid positions. SNAP-25A is predominantly expressed in the embryonic brain whereas SNAP-25B dominates in the adult brain [23]. The physiological significance behind the differential expression is not known. Synaptobrevin [24] in neurons is predominantly found on synaptic vesicles where it is anchored with the help of a transmembrane domain. Like syntaxin, it contains a single SNARE motif (Fig. 2) and there are also two synaptobrevin isoforms, synaptobrevin 1 and synaptobrevin 2, which are differentially distributed in the nervous system.

Syntaxin 1

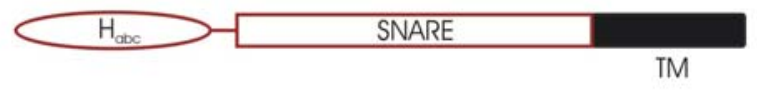

SNAP-25

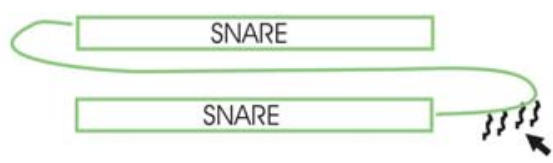

Synaptobrevin

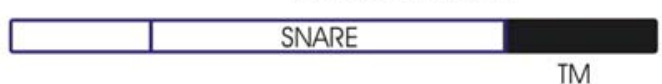

Figure 2 Domain structure of neuronal SNAREs

Syntaxin 1 contains a C-terminal transmembrane domain (TM), SNARE motif, and $\mathrm{H}_{\mathrm{abc}}$ domain, which is an anti-parallel three helical bundle with a left handed twist (for the structure of $\mathrm{H}_{\mathrm{abc}}$ domain, refer fig 1). The $\mathrm{H}_{\mathrm{abc}}$ domain is linked to the SNARE motif by a flexible linker. SNAP-25 has two SNARE motifs that are connected by a flexible linker, the palmitoylation sites are indicated by the arrow. Synaptobrevin is C-terminally anchored with the help of a transmembrane domain. It has a single SNARE motif and contains flexible amino acid residues at the extreme Nterminus. 
The SNARE motifs of these neuronal SNAREs assemble to form a parallel four helical coiled-coil bundle, which is called the ternary complex [25] (Fig. 3). Syntaxin and synaptobrevin contribute one helix and SNAP-25 contributes two helices. The first and the fourth amino acid residue of the heptad repeat point towards the center of the helix and lie perpendicular to the axis of the helix bundle. Four interacting amino acid residues each from one helix of the four helical bundle constitute a layer. The helix bundle is largely stabilized by these interactions.

There are altogether 16 layers and the central layer (zero layer), which contrary to all other layers is not hydrophobic and contains one-arginine (R) and three glutamine (Q) residues. The three-glutamine residues are provided by syntaxin and SNAP-25 and the arginine residue is provided by the synaptobrevin. Based on this observation the SNAREs were reclassified as Q- and R- SNAREs [26].

The Q-SNAREs were further classified into Qa,Qb and Qc SNAREs [27]. The Qa SNAREs corresponds to syntaxin 1 homologues, Qb to SNAP-25 helix 1 homologues and Qc to SNAP-25 helix 2 homologues. It was proposed that a fusion-competent SNARE complex must contain Qa, Qb, Qc, and R combination. It is more convenient to tag SNAREs as Qa, Qb, Qc or R category than to tag them as v- or t-SNAREs. The vand t- SNARE classification is not precise as t-SNAREs can be found on vesicles and vice versa. Moreover the v- and t- SNARE classification cannot be used in homotypic vesicular fusion events. In such events structurally and functionally similar vesicles fuse making it impossible to assign a vesicle and target. The structural classification into Qand R-SNAREs reduces this ambiguity.

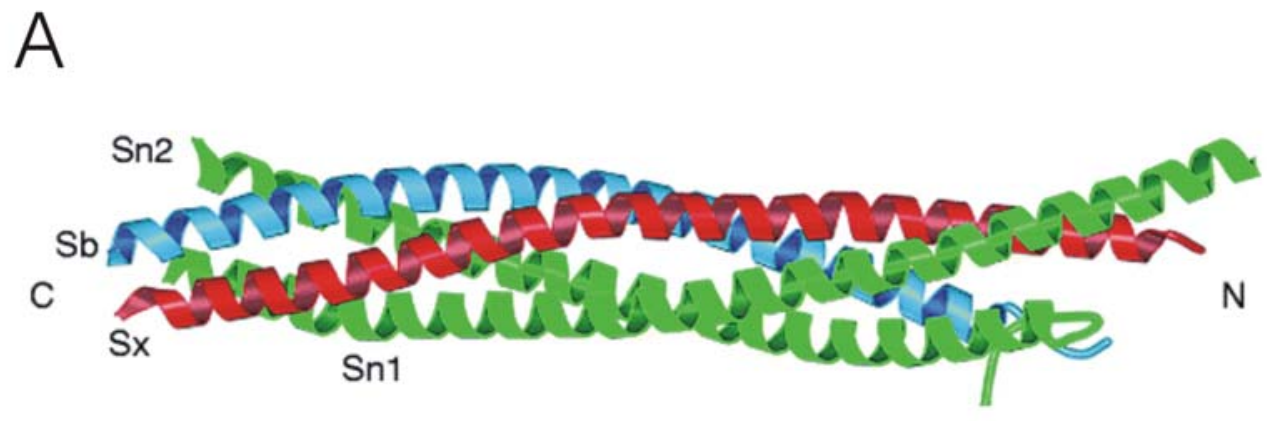


B

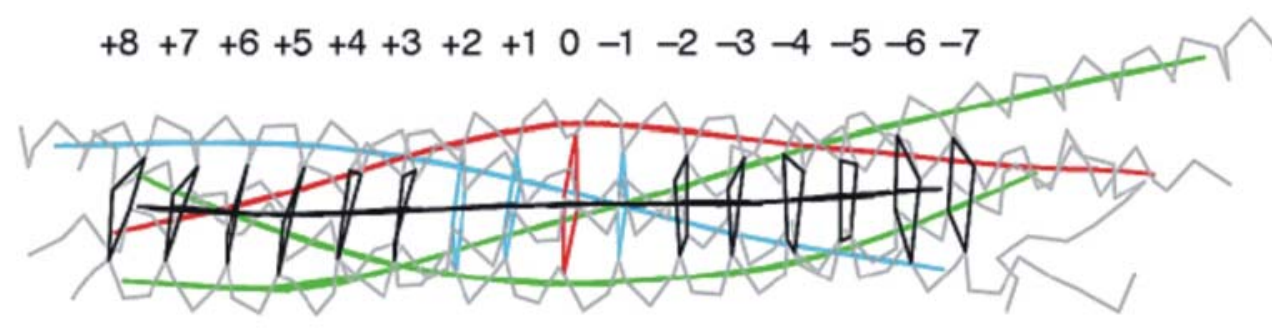

Figure 3 Structure of the neuronal SNARE complex

(A). The SNARE motifs of the neuronal SNAREs interact to form a parallel four helical coiledcoil bundle, it comprises syntaxin 1a (Sx), first SNARE helix of SNAP-25A (Sn1), second SNARE helix of SNAP-25A (Sn2) and synaptobrevin2 (Sb). The N and $\mathrm{C}$ terminus of the bundle is labeled. (B). The neuronal SNARE complex showing the layers -7 to +8 ; 0 layer in red, $1,+1,+2$ in blue and all the other layers in black. (The figure was modified from Sutton RB et al, 1998, Nature).

\subsection{The neuronal SNARE assembly}

In order to understand the mechanism of neurotransmitter release it is important to understand how the putative fusion factors, the neuronal SNAREs, assemble. The Zipper model gives the simplest explanation of the process $[28,29]$. It assumes that the assembly of synaptobrevin, which is on the synaptic vesicle with syntaxin and SNAP-25, which are on the presynaptic plasma membrane, initiates N-terminally. This zippering would bring the synaptic vesicle in juxtaposition with the plasma membrane and it could eventually cause fusion. This model gained large support from subsequent structural, biochemical, biophysical and physiological studies. However the zipper model is largely based on circumstantial evidence.

The interaction between neuronal SNAREs is largely through their SNARE motifs. The SNARE motifs of these SNAREs are largely unstructured in solution, but transform into a $\alpha$-helical structure after interaction [30]. This transformation probably 
releases enough energy to overcome the repulsion between membranes and may finally cause membrane fusion.

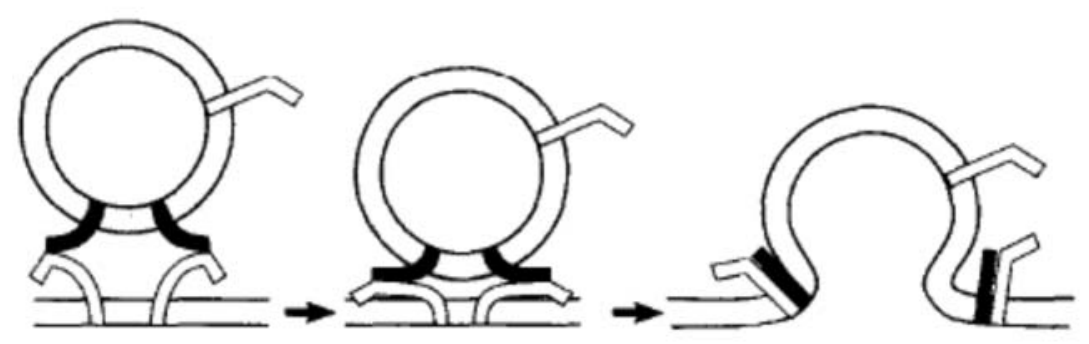

\section{Figure 4 Zipper model}

Putative mechanism of neurotransmitter release as per the zipper model. The assembly of synaptobrevin (black molecule) from the vesicle with syntaxin and SNAP-25 (white molecule) on the presynaptic plasma membrane could bring the synaptic vesicle in juxtaposition with the plasma membrane and could finally cause fusion. For simplicity only one of the Q-SNAREs is shown. (Figure adapted from Hanson PI et al, 1997, Curr Opin Neurobiol)

Syntaxin1 plays an important role by initiating neuronal SNARE assembly. Syntaxin1 exists in two conformations which are termed as 'open' and 'closed' (Fig. 5). In the closed conformation the $\mathrm{H}_{\mathrm{abc}}$ domain of syntaxin interacts intramolecularly with the N-terminal portion of the SNARE motif and prevents its association with the other neuronal SNAREs. In the open conformation the $\mathrm{H}_{\mathrm{abc}}$ domain does not interact with the SNARE motif and thereby syntaxin can interact with its partners. Syntaxin can freely switch between the two conformational states [31]. In solution, the conformational switch affects the rate of neuronal SNARE assembly [31], syntaxin lacking the $H_{a b c}$ domain, which resembles syntaxin in the open conformation, is able to form a ternary complex (complex with SNAP-25 and synaptobrevin) at a faster rate ( 10 times) than the full length syntaxin [31]. In line with this observation, the cleavage of $\mathrm{H}_{\mathrm{abc}}$ domain hastens the SNARE mediated fusion [32] suggesting that faster assembly leads to faster fusion. 
Genetic experiments in C.elegans have uncovered several proteins that play a role in exocytosis [33]. One of the protein is unc-18, its mammalian homologue Munc-18 [34, 35] has been shown to form a stable complex with syntaxin1a and stabilize the closed conformation of syntaxin [36] (Fig. 5). At least in vitro such a complex prevents syntaxin from interacting with the other neuronal SNAREs, and has been proposed to negatively regulate assembly [37]. On the other hand, genetic ablation of Munc-18 abolishes neurotransmitter release contradicting the proposed negative regulatory role [38]. Thus it is still ambiguous whether Munc-18 plays a negative regulatory role in exocytosis.

Munc-18 stabilises syntaxin in the 'closed' conformation and proteins like tomosyn [39] and Munc-13 [40, 41] could possibly release Munc-18 and switch the syntaxin to an 'open' conformation. Therefore syntaxin either by itself [31] or with the help of protein-protein interactions switches between two conformational states. A switch from closed to open conformation favours SNARE assembly but it is not clear if such a switch is physiologically relevant. In solution, uncomplexed syntaxin, regardless of $H_{a b c}$ domain/conformation switch, assembles with its complementary neuronal SNAREs. 


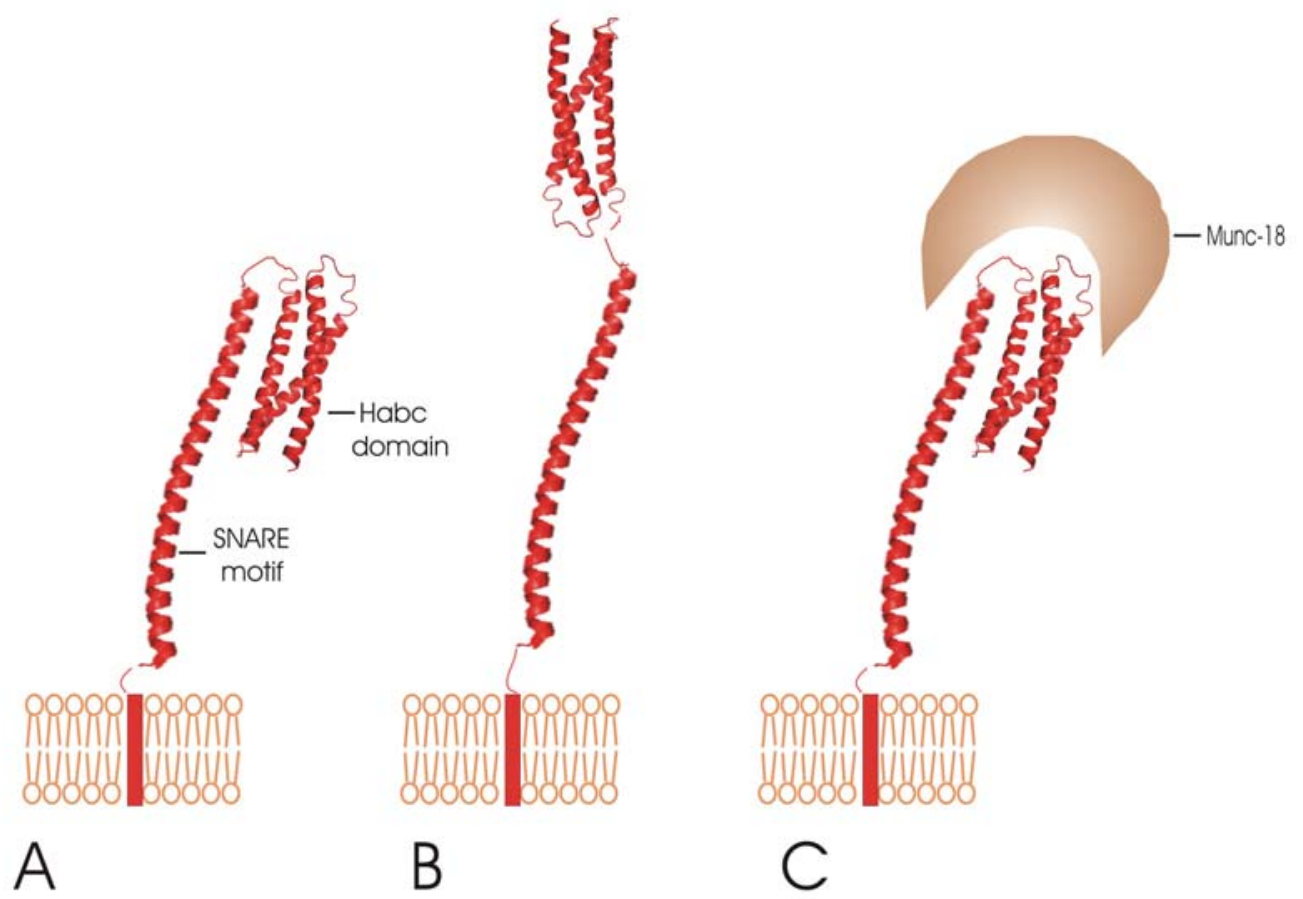

\section{Figure 5 Syntaxin conformational switch}

(A). Syntaxin in 'closed' conformation; the $\mathrm{H}_{\mathrm{abc}}$ domain of syntaxin interacts with the SNARE motif and prevents syntaxin from assembling with its complementary SNAREs viz SNAP-25 and synaptobrevin. (B). Syntaxin in 'open' conformation; the $\mathrm{H}_{\mathrm{abc}}$ domain does not interact with the SNARE motif and the SNARE motif is free to pair with SNAP-25 and synaptobrevin. (C). Closed conformation of syntaxin stabilised by Munc-18, syntaxin cannot pair with its complementary SNAREs in this conformation.

\subsubsection{Intermediate step in neuronal SNARE assembly}

Hysteresis is observed during the thermal denaturation and renaturation of the neuronal SNARE complex. The neuronal SNARE complex disassembles at a very high temperature (around $82^{\circ} \mathrm{C}$ )[42, 43]. Upon decreasing the temperature, the disassembled monomers reassemble at a $20^{\circ} \mathrm{C}$ lower temperature (around $63^{\circ} \mathrm{C}$ ). This difference in the folding and unfolding transition (hysteresis) appears to be a hallmark of all SNARE complexes. This difference suggests that the neuronal complex does not dissociate spontaneously and the assembly probably proceeds through a less stable intermediate. As the complex is not in an equilibrium it is a prerequisite to have an ATPase like NSF [44] to disassemble the complex so that SNAREs could be reused for fusion. 


\subsubsection{The assembly pathway}

The SNARE motifs of syntaxin and SNAP-25 interact transiently (1:1 dimer) and initiate the SNARE assembly [45] and this is the intermediate in the neuronal SNARE assembly pathway. The N-terminal SNARE residues of syntaxin and SNAP-25 are absolutely essential for the formation of this dimer. The formation of this intermediate, the transient 1:1 dimer, is the rate-limiting step in neuronal SNARE assembly with a second order rate constant of about $7000 \mathrm{M}^{-1} \mathrm{~S}^{-1}$ [45]. It has been suggested that this dimer offers an open binding site for synaptobrevin and the assembly culminates in the ternary complex (syntaxin, SNAP-25 and synaptobrevin in 1:1:1 stoichiometry). This pathway is referred to as the 'on-pathway' (Fig. 6) and a detailed kinetic analysis of this pathway will lead to a better understanding of the mechanism of neurotransmitter release. However, it has been difficult to evaluate the binding mode of synaptobrevin to the syntaxin-SNAP-25 dimer as the synaptobrevin binding site is readily occupied by a second syntaxin, resulting in a binary complex (2:1, syntaxin, SNAP-25 complex)[30]. The second syntaxin has to dissociate from the complex to enable synaptobrevin binding. As synaptobrevin cannot actively displace the second syntaxin from the 2:1 complex, this pathway is referred to as the 'off-pathway' (Fig. 6). It is very probable that physiologically, the neuronal SNAREs like yeast exocytotic SNAREs [46] assemble via the 'on-pathway'.

As mentioned above, due to the 'off-pathway' it has been difficult to evaluate the binding mode of the synaptobrevin to syntaxin-SNAP-25 dimer. However there are certain observations that indirectly suggest that synaptobrevin could bind to the Nterminus of the syntaxin-SNAP-25 dimer. For instance, a structured N-terminus is observed in Sso1/Sec9 complex, a yeast homologue of syntaxin/SNAP-25 complex [46]. It is suggested that Snc1/2p a homologue of synaptobrevin could bind to this $\mathrm{N}$-terminus to initiate zippering. This is in line with the proposed zipper model for SNARE assembly [28]. Such an N-terminal assembly is topologically relevant, syntaxin and synaptobrevin are type II membrane proteins and their N-terminal assembly would bring the synaptic vesicle in close proximity to the presynaptic plasma membrane. There is also other supportive evidence for the zipper model and N-terminal assembly: (1) Threonines and 
serines are frequently encountered in otherwise mostly hydrophobic layers of SNARE complexes and they can form intermolecular hydrogen bonds and their formation could assist a directed assembly process [47]. (2) Deuterium exchange experiments have shown that the N-terminal half of the neuronal SNARE complex is relatively more stable than the C-terminal half. [48]. (3) A monoclonal antibody that recognizes the N-terminal portion of SNAP-25 prevents SNARE assembly [49]. (4) N-terminal truncations of syntaxin or SNAP-25 abolishes SNARE assembly [45].

As a whole, it is suggested that the transient N-terminal association of syntaxin and SNAP-25 (1:1 dimer) initiates SNARE assembly. Synaptobrevin probably binds to this intermediate at the $\mathrm{N}$-terminus to then zip further towards the $\mathrm{C}$ terminus. The formation of this 1:1 intermediate is the rate limiting step and in vivo it is probably catalyzed and/or stabilized by other factors.

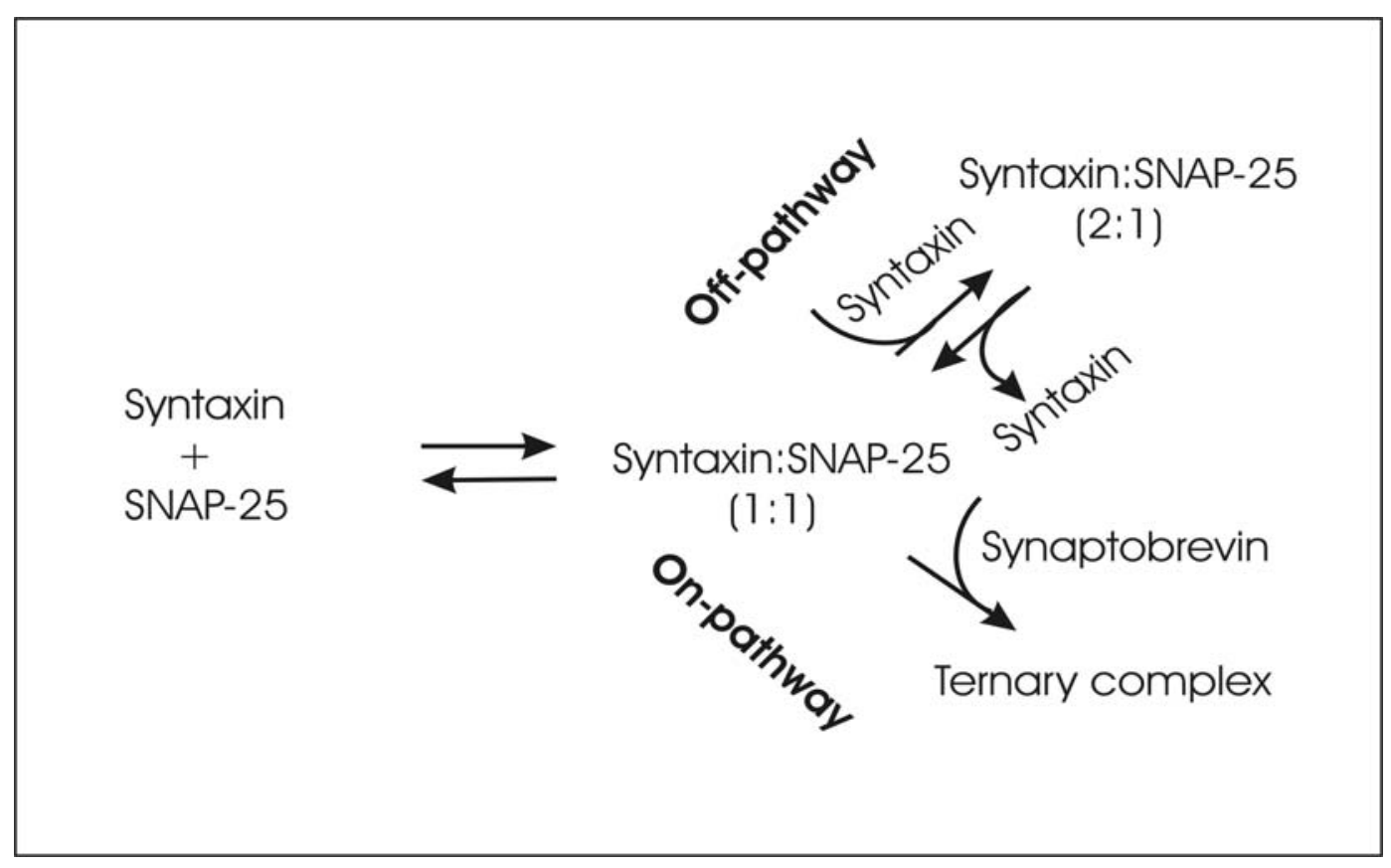

Figure 6 Current model of SNARE assembly pathway

Assembly of neuronal SNAREs is thought to initiate with N-terminal transient interaction of syntaxin and SNAP-25 SNARE motifs. Synaptobrevin binds to this transient dimer and forms a ternary complex. This pathway is referred as 'On pathway'. The binding site of synaptobrevin can also be occupied by another syntaxin resulting in a binary complex which contains syntaxinSNAP-25 in 2:1 stoichiometry. This pathway is referred to as the 'off pathway'. The second syntaxin should leave the complex to allow for synaptobrevin binding. 


\subsubsection{Status of SNAREs in neuronal exocytosis}

Genetic ablation of neuronal SNAREs leads to a block in exocytosis [50-52], furthermore, proteolytic cleavage of neuronal SNAREs by specific neurotoxins or mutations in SNAREs [53-55] alters the exocytotic behavior of secretory cells [56-60], suggesting that SNAREs are an essential part of the exocytotic machinery. Any alteration in the availability of SNAREs changes the number of vesicles that are being kept in the 'release-ready' state [61-64] suggesting that it is through SNAREs the vesicles are kept in that state. The crucial question in the field is whether they catalyze rapid reactions like fast neuronal exocytosis. Neuronal SNAREs induce fusion when reconstituted in liposomes however a single round of fusion takes several minutes to complete [32, 65]. This suggests that neuronal SNAREs may not catalyze a rapid reaction like neuronal exocytosis. However it is inappropriate to disregard the catalyzing role of SNAREs based on this observation. Biochemical analysis indicates that unproductive side reaction like the 'off-pathway' occurs during the neuronal SNARE assembly. It is possible that the sluggishness in liposome fusion is due to such side reactions.

Alternatively, SNAREs could be kept in a specific configuration to accomplish rapid fusion. Few studies suggest that before $\mathrm{Ca}^{2+}$ influx the trans-SNARE complex is kept in a partially zippered state (Fig. 7A), where only the N-terminal portions of SNAREs are assembled [49, 66, 67]. It has been proposed that after the entry of $\mathrm{Ca}^{2+}$ neuronal SNAREs can completely zipper and drive exocytosis. This model is based on the outcome of physiological studies carried out on neurons and neurosecretory cells, which were aimed at dissecting the assembly pathway in vivo and to evaluate the configuration of SNAREs during different stages of exocytosis. The evidence about the existence of partially zippered SNARE complexes is only indirect. These studies monitored the alteration of the exocytotic behavior of cells after infusion of SNARE specific toxins or antibodies and from this drew conclusions about the presumable state of SNARE complexes. For example infusion of Botulinum/B neurotoxin light chain (BoNT/B-LC) in crayfish neurons blocks exocytosis before stimulation, whereas infusion 
of tetanus toxin light chain (TeNT-LC) does not [66]. Both the toxins cleave synaptobrevin at the same site but BoNT/B-LC requires the C-terminus of synaptobrevin for recognition and TeNT-LC requires the N-terminus. Furthermore, it is known that the toxins cannot act when the SNAREs are engaged in a complex [68], as only BoNT/B-LC blocked exocytosis it was proposed that SNAREs are probably N-terminally assembled or partially zippered. It is a very attractive mechanism to maintain vesicles in a `releaseready' state however the evidence is indirect and it remains to be determined if SNAREs can actually reside in such a configuration.

There are also hypotheses that disregard a late role for SNAREs in fusion. For instance it has been proposed that before $\mathrm{Ca}^{2+}$ influx SNAREs might reside in a completely zippered state and cause hemifusion, a fusion intermediate where the outer layers of the presynaptic plasma membrane and synaptic vesicle are fused [69]. Synaptotagmin 1 (for reviews, [69, 70]), the $\mathrm{Ca}^{2+}$ sensor in fast neuronal exocytosis, was assumed to bind to the fully-assembled SNAREs (Fig. 7B). After the $\mathrm{Ca}^{2+}$ influx synaptotagmin 1 with the help of $\mathrm{Ca}^{2+}$-dependent phospholipid interaction [71-73] might cause the inner leaflet merger. This is also an attractive hypothesis as it accounts for the speed of exocytosis, here after the $\mathrm{Ca}^{2+}$ influx the synaptotagmin just has to shift its binding partners to cause fusion. However, as mentioned above, it remains to be determined if SNAREs exist in such a configuration.

Taken together, SNAREs are potential candidates to cause fusion, it is still unresolved if they catalyze a rapid reactions like neuronal exocytosis. 
A

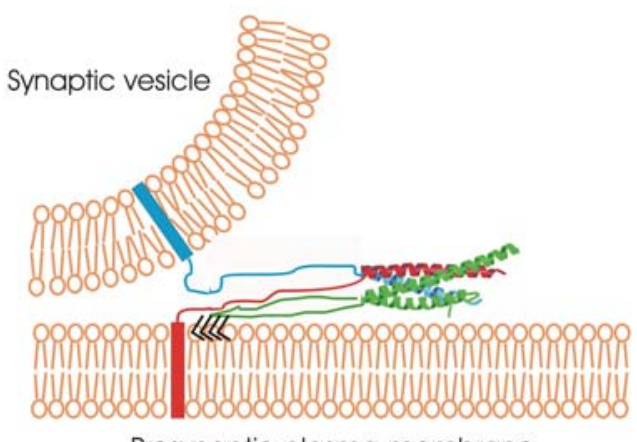

Presynaptic plasma membrane
B

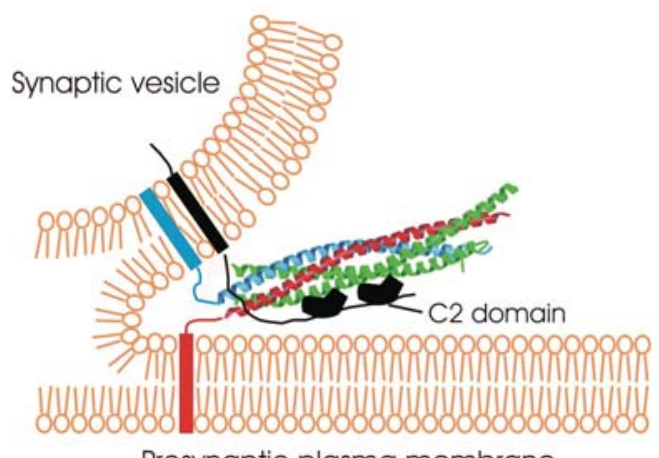

Presynaptic plasma membrane

Figure 7 Partially and fully assembled SNAREs

A hypothetical model depicting partially and fully-assembled SNAREs (A) N-terminally assembled neuronal SNAREs, syntaxin (red), SNAP-25 (green), synaptobrevin (blue). The SNARE complex that bridges two membranes is called the 'trans-complex'. (B) SNAREs are fully assembled; the zippering might cause the merger of outer leaflets of synaptic vesicle and presynaptic plasma membrane. Synaptotagmin (black) with two C2 domains bound to SNAREs is shown. After the entry of $\mathrm{Ca}^{2+}$ synaptotagmin could bind to phospholipids and cause the merger of inner leaflets.

\subsection{SNARE regulators}

The core fusion machinery, which consists of the neuronal SNAREs is regulated by several proteins.

\subsubsection{Tomosyn}

Tomosyn is one of the regulating proteins; it is a soluble protein of $130 \mathrm{kDa}$ and contains an N-terminal domain with WD40 repeats and a C-terminal R-SNARE (synaptobrevin like) motif. Tomosyn was isolated as a binding partner of syntaxin1a from rat brain cytosol in a GST-pull down assay [39]. The N-terminal domain of tomosyn is homologous to the Drosophila tumor suppressor lethal giant larvae (Lg1)[74] and its mammalian homologue MglI [75] and to the yeast proteins Sro7p and Sro77p [76]. The Lgl proteins play an important role in maintaining cell polarity (for reviews $[77,78]$ ). In vertebrates, tomosyn has two paralogues, Tomosyn 1 and 2 and seven splice variants [79, 80]. Surprisingly, all the splice variants differ only at the region between the WD40 repeats and the R-SNARE motif and their functional relevance is not yet known. 
The R-SNARE motif of tomosyn is very similar to that of synaptobrevin. Like synaptobrevin, it interacts with syntaxin and SNAP-25 and forms a ternary complex [81, 82]. This ternary complex, like the synaptobrevin containing ternary complex, exhibits hysteresis and can be disassembled by NSF. As outlined above, tomosyn is a binding partner of syntaxin1a [39]. As high concentrations of the tomosyn SNARE motif are required to prevent full-length tomosyn and syntaxin interaction [79], it is likely that the tomosyn interaction with syntaxin is not just confined to its SNARE motif. However, the interactions of region N-terminal to the SNARE motif or the interacting partners of the tomosyn $\mathrm{N}$-terminal domain are not known.

Functionally, tomosyn was proposed to be a positive regulator of exocytosis [39]. Tomosyn is shown to release Munc-18 from the tight Syntaxin-Munc-18 complex (see Fig. 5); by releasing Munc-18 it might facilitate the formation of the neuronal SNARE complex. Another indication for the proposed positive regulatory function comes from RNAi experiments in SCG (superior cervical ganglion) neurons [83], which showed that knocking down tomosyn decreased the number of release-competent vesicles.

In contrast, several other studies suggested Tomosyn as a negative regulator of exocytosis. Overexpression of tomosyn in PC12 cells inhibited $\mathrm{Ca}^{2+}$ dependent exocytosis [39]. Another study that analyzed the release of secretory granules from PC12 membrane sheets, also found a reduction in exocytosis after tomosyn overexpression [81]. Similarly, in adrenal chromaffin cells overexpression of tomosyn reduced the number of fusion competent vesicles [84]. Overexpression of tomosyn also inhibited insulin stimulated fusion of GLUT4 containing vesicles [85]. Tomosyn has also been reported to guide the fusion of plasmalemmal precursor vesicles by localizing to the palm of the neuronal growth cone [86].

The lack of transmembrane anchor makes the tomosyn SNARE complex `fusioninactive'. Tomosyn competes with 'fusion-active' synaptobrevin for the same binding partners, therefore tomosyn can act as a negative regulator of exocytosis. The tomosyn SNARE complex probably needs to be disassembled by the ATPase NSF to allow the QSNAREs to engage with vesicular synaptobrevin. 
The activity of tomosyn is regulated, phosphorylation of tomosyn by cAMPdependent protein kinase A reduces the affinity of tomosyn to syntaxin 1 [83]. It appears that there are several other regulatory mechanisms that are to be unraveled. For example, in adrenal chromaffin cells the inhibition caused by the overexpression of tomosyn could be partially relieved by elevating calcium levels [84] and the underlying molecular mechanism is not known. In addition, proteins that interact with the N-terminal domain of tomosyn are yet to be identified. Possibly this region of tomosyn, similar to Lgl-like proteins, is linked to a signaling pathway. Therefore more research is needed to understand the precise role of tomosyn.

\subsubsection{Amisyn}

Amisyn is another SNARE regulatory protein, it is a soluble protein of $25 \mathrm{kDa}$ and like tomosyn, and it contains an N-terminal domain and a C-terminal R-SNARE motif. The N-terminal domain of amisyn is similar to the C.elegans homologue of the tethering protein Uso1 [87] and also has limited homology ( $~ 32 \%$ ), to a Sec3-like protein (Sec3 is also implicated in tethering). Amisyn is enriched in the brain and it coimmunoprecipitates with syntaxin 1a [88]. Like tomosyn, the SNARE motif of amisyn forms a ternary complex with the neuronal SNAREs, syntaxin1a and SNAP-25 [88]. Orthologues of Amisyn are found only in fishes, birds and mammals. Amisyn lacks a membrane anchor but a considerable amount was found in the membrane fraction [88]. Therefore amisyn could perhaps interact with the cytoskeletal network or could be attached to the membrane by lipid modifications; however it lacks recognizable lipidation motifs. Alternatively amisyn could be indirectly attached to the membrane because of its interaction with the membrane protein syntaxin 1a.

The SNARE motif of amisyn does not considerably inhibit the norepinephrine secretion from permeabilised PC12 cells [89]. This suggests that unlike synaptobrevin, the SNARE motif of amisyn is not efficient in inhibiting exocytosis [88]. However, contrary to the SNARE motif, the full-length amisyn inhibits the secretion of growth hormone from PC12 cells. Amisyn also forms 'fusion-inactive' SNARE complex with 
syntaxin and SNAP-25 and inhibits exocytosis but surprisingly, the inhibition is not due to its binding to syntaxin [89]. Amisyn appears to have a unique regulatory function in exocytosis and very little is known about amisyn, which makes it is necessary to characterize amisyn in greater detail.

\subsection{Aims of this study}

Neurotransmitter release is a rapid and highly regulated form of membrane fusion. As in all membrane fusion events, SNARE proteins play an important role in neurotransmitter release. Biochemical studies played an important role in deciphering the function of SNARE proteins. SNAREs are the minimal cellular machinery that is required for fusion. It is shown that neuronal SNAREs mediate fusion when reconstituted into liposomes. However, intriguingly, the fusion occurs in the order of minutes, whereas physiologically, neurotransmitter release occurs in a millisecond time scale. Although it appears that SNAREs cannot mediate rapid fusion reactions, it is still possible that there might be unproductive side reactions that hinder SNARE mediated fusion rate in the liposome fusion experiments. Alternatively, under physiological conditions SNAREs could be kept in a specific configuration to accomplish rapid fusion. The aim of this study is to explore these possibilities using structural, biochemical and biophysical methods, which give direct access to the SNAREs. I plan to evaluate SNARE mediated fusion under various assembly conditions and configurations in order to better understand the molecular mechanism of neurotransmitter release.

Furthermore, several proteins interact with the neuronal SNAREs and regulate neurotransmitter release, but the precise molecular mechanism is largely unknown. Amongst those regulatory proteins are the two proteins tomosyn and amisyn. Both are soluble proteins and possess an N-terminal domain and a C-terminal synaptobrevin like, R-SNARE motif. I plan to use the above mentioned methods in order to understand how these two proteins influence the activity of SNAREs. 


\section{Materials and Methods}

\subsection{Materials}

\subsubsection{Chemicals}

Acrylamide/Bisacrylamide solution, Roth (Karlsruhe, Germany)

Agarose (low EEO), Applichem (Darmstadt, Germany)

Ammoniumpersulfate (APS), Sigma (Deisenhofen, Germany)

Bradford-Reagent, Biorad (Richmond, USA)

Calcium chloride dihydrate, Fluka (Switzerland)

Coomassie Brilliant Blue R-250, Serva (Heidelberg, Germany)

Dipotassium hydrogen phosphate, Merck (Darmstadt, Germany)

Disodium hydrogen phosphate, Merck (Darmstadt, Germany)

Dithiothreitol (DTT), Roth (Karlsruhe, Germany)

EDTA Titriplex III, Merck (Darmstadt, Germany)

Glycine, Sigma (Deisenhofen, Germany)

HEPES (N-(2-hydroxyethyl) piperazine - N'-2-ethanesulphonicacid), GERBU (Gaiberg,

Germany)

Imidazole, Sigma (Deisenhofen, Germany)

Isopropyl- $\beta$-D-thiogalactoside (IPTG), Roth (Karlsruhe, Germany)

Magnesium chloride, Sigma-Aldrich (St.Louis, USA)

MPD (2-methyl-2, 4 pentanediol), Fluka (Switzerland)

PMSF (paramethyl sulphonyl fluoride), Roth (Karlsruhe, Germany)

Potassium dihydrogen phosphate, Merck (Darmstadt, Germany)

Protease K, Boehringer (Mannheim, Germany)

Sodium hydrogen carbonate, Merck (Darmstadt, Germany)

Sodium chloride, Merck (Darmstadt, Germany)

Sodium cholate, Sigma-Aldrich (St.Louis, USA)

Sodiumdodecylsulfate (SDS), Biorad (Richmond, USA)

TEMED ( $\mathrm{N}^{\prime}, \mathrm{N}^{\prime}, \mathrm{N}^{\prime}, \mathrm{N}^{\prime}$ - Tetramethylethylenediamine), Biorad (Richmond, USA) 
Tricine, Applichem (Darmstadt, Germany)

Tris (hydroxymethyl)-aminomethane (Tris), Merck (Darmstadt, Germany)

Triton X-100, Merck (Darmstadt, Germany)

Tween - 20, DAKO (Carpinteria, USA)

Urea, Merck (Darmstadt, Germany)

\subsubsection{Chemical solutions}

Acetic acid, Merck (Darmstadt, Germany)

Hydrochloric acid, Merck (Darmstadt, Germany)

Isopropanol, Merck (Darmstadt, Germany)

Methanol, Merck (Darmstadt, Germany)

\subsubsection{For bacterial culture}

Tryptone, Roth (Karlsruhe, Germany)

Yeast extract, Roth (Karlsruhe, Germany)

Agar-agar, Roth (Karlsruhe, Germany)

LB (Luria Bertani) media: 10g tryptone, $5 \mathrm{~g}$ yeast extract and $5 \mathrm{~g} \mathrm{NaCl}$, add 1 liter with distilled water added $12 \mathrm{~g}$ agar for solid media.

TB (Terrific broth) media: 13.3g tryptone, $26.7 \mathrm{~g}$ yeast extract, $4.4 \mathrm{ml}$ glycerol.

TB salt: $0.17 \mathrm{M} \mathrm{KH}_{2} \mathrm{PO}_{4}, 0.72 \mathrm{M} \mathrm{K}_{2} \mathrm{HPO}_{4}$.

Before inoculation, the media were autoclaved and brought to room temperature. In the case of TB media, after autoclaving, TB salt was added.

\subsubsection{Bacteria stains}

The following strains were obtained from (Stratagene, La Jolla, CA, USA)

Escherichia coli BL21 (DE3) B F- dcm ompT hsdS (rB- mB-) gal $\lambda$ (DE3)

Escherichia coli XL-1-Blue recA1, endA1, gyrA96, thi-, hsdR17, (rk-, mk+), supE44, relA1, lac-, [F', traD36, proAB, lacIqZ $\Delta \mathrm{M} 15, \mathrm{Tn} 10$ (tetr)] 


\subsubsection{Enzymes}

Restriction endonucleases, New England Biolabs (Ipswich, USA) or MBI Fermentas (Ontario, Canada)

Lyzozyme, Applichem (Darmstadt, Germany)

DnaseI, Applichem (Darmstadt, Germany)

Thrombin, Merck (Darmstadt, Germany)

\subsubsection{Fluorescent labels}

Oregon Green ${ }^{\circledR} 488$ iodoacetamide, Invitrogen (USA)

Texas Red ${ }^{\circledR} \mathrm{C}_{5}$ bromoacetamide, Invitrogen (USA)

Alexa Fluor® $488 \mathrm{C}_{5}$ maleimide, Invitrogen (USA)

\subsubsection{Cuvettes}

Fluorescence cell with magnetic stirrer, with a path length of 10 x 4 mm (Hellma)

For CD measurements: 1mm path length cuvettes (Hellma) or $10 \mathrm{x} 10 \mathrm{~mm}$ path length (Hellma) (For CD kinetics).

\subsubsection{Instruments}

ÄKTA explorer, Amersham Pharmacia Biotech (Uppsala, Sweden)

UV-spectrophotometer, UV-2401 PC Shimadzu (Japan)

Luminescent image analyzer, LAS-1000 Fujifilm (Japan)

Fluoromax-2, Jobin Yvon-Spex (Edison, NJ, USA)

Flurolog, Jobin Yvon-Spex (Edison, NJ, USA)

Spectroplarimeter J-720, Jasco (CD-spectroscopy)

\subsubsection{Softwares}

Kaleidagraph, synergy software

Corel draw 8, Corel Corporation

Endnote 9, Thomson 


\subsubsection{Constructs}

The SNARE proteins used in the study were syntaxin 1a, SNAP-25A and synaptobrevin2. Norway rat (Rattus norvegicus) cDNAs, made from the transcript encoding these proteins, were used to make the expression constructs.

The following expression constructs were used,

Syntaxin variants: H3 domain of syntaxin1a (residues 180-262) [90], the syntaxin with the transmembrane region (residues 183-288)[91].

SNAP-25A variants: Cysteine less variant of SNAP-25A (residues 1-206) [90]

Synaptobrevin variants: Synaptobrevin (residues 1-96) [90], synaptobrevin (residues 1116) [91]. The truncated synaptobrevins, synaptobrevin (25-96), synaptobrevin (35-96), synaptobrevin (42-96), synaptobrevin (1-70) and synaptobrevin (1-65) were generated by PCR amplication from synaptobrevin (1-96) construct [45]. All the truncated synaptobrevins were cloned in pET 28 expression vector (Novagen).

Labeling: The following single cysteine mutants synaptobrevin (1-96 cys 28), synaptobrevin (42-96 cys 61), syntaxin (183-262 cys 225) [92] syntaxin (183-262 cys 197) [92] were used. Complexin 1 containing a single cysteine at position 39 was used [93].

Crystallization: Syntaxin 1a (residues 188-259), the first SNARE helix of SNAP-25A (Sn1, residues 7-83), the second helix of SNAP-25A (Sn2, residues 142-204), the RSNARE helix of synaptobrevin 2 (residues 30-89). A shortened fragment of the SNARE complex-forming domain of tomosyn was constructed (residues 1050-1108, numbering according to the m-splice variant of tomosyn1). Due to an erroneous DNA primer a frame shift at the very $\mathrm{COOH}$ terminus of the tomosyn fragment occurred, which added 32 amino acids of vector-derived sequence. However, this was only detected after crystallization of the tomosyn SNARE complex. These residues were probably unstructured in the tomosyn SNARE complex and were not detected in the crystal structure [82].

Toxins: Botulinum neurotoxin D light chain construct was kindly provided by H.Niemann (medizinische hochschule Hannover, Germany).

Endobrevin: Rat endobrevin (residues 1-100) was used [94]. 
Tomosyn: For the spectroscopic investigations tomosyn fragment (residues 1051-1116) was used. The numbering is according to the m-splice variant of tomosyn.

Complexin: Complexin 1 (residues 1-134) was used [93].

Amisyn: Amisyn clone was kindly provided by Prof. Thomas weimbs (UCAL, Santa Barbara). Full length Amisyn (residues (1-210) was amplified using the primers (For: 5'AAA AGA ATT CCA TGA ATT CAC TGC GTG CTG TC-3’; Rev: 5'- CGC TAC GCC GGC GTC AAC ATT TGT GCT TCA TGG-3’) and was cloned in pET 28a vector after restriction digestion with NdeI and EagI. For the SNARE motif the forward primer (5'- AAA AGA ATT CCA TGG GAG GAA ACA GCA TCC TC-3’) and the reverse primer as in full length amisyn was used; The SNARE motif of amisyn, Ami-SN (residues 162-210) was cloned in pET 28a after restriction digestion with NdeI and EagI. The Amisyn constructs were sub cloned by our technician Maria Druminski.

\subsection{Methods}

\subsubsection{E.coli competent cells}

The electro-competent cells were made through the following procedure; $10 \mathrm{ml}$ of overnight grown E.coli culture was added to $1 \mathrm{~L} \mathrm{LB}$ medium. The culture was grown at $37^{\circ} \mathrm{C}$ in a shaker until an O.D. of about 0.5 - 0.7 was reached. The cells were later pelleted and resuspended for two consecutive times in $1 \mathrm{~L}$ and $500 \mathrm{ml}$ ice-cold HEPES buffer (1mM HEPES, pH-7.0) respectively. The cells were again pelleted and were resuspended in 20ml HEPES buffer with $10 \%(\mathrm{v} / \mathrm{v})$ glycerol followed by pelleting and resuspension in $3 \mathrm{ml} \mathrm{10 \%} \mathrm{(v/v)} \mathrm{glycerol.} \mathrm{They} \mathrm{were} \mathrm{dispensed} \mathrm{into} 50 \mu \mathrm{l}$ aliquots and frozen rapidly in liquid nitrogen. The cells were later stored at $-80^{\circ} \mathrm{C}$.

The heat-competent cells were made as described in [95].

\subsubsection{Transformation}

Electroporation: The electrocompetent cells were thawed on ice. To it 20ng of plasmid DNA was added and was kept on ice for $10 \mathrm{~min}$. The cells were then transferred to a $0.2 \mathrm{~cm}$ electroporation cuvette (Bio-Rad). The electroporation was carried out in a 
gene pulser (Bio-Rad) with $25 \mu \mathrm{F}, 1.8 \mathrm{kV}$, and 200 ohms pulse. After electroporation $800 \mu \mathrm{LB}$ medium was added to the cuvette. The cells were transferred to a microfuge tube and were grown at $37^{\circ} \mathrm{C}$ for $40 \mathrm{~min}$ in a shaker. Then $50 \mu \mathrm{l}$ of the culture was plated on an LB agar plate that contains the appropriate antibiotic for selecting the transformed cells. The plates were kept overnight at $37^{\circ} \mathrm{C}$ to get transformed colonies.

Heat-shock: An aliquot of the heat competent cells was thawed on ice and to it 30ng plasmid DNA was added. It was incubated for $20 \mathrm{~min}$ on ice. The cells were then subjected to heat shock by keeping them for $90 \mathrm{~s}$ in a water bath that was kept at $42^{\circ} \mathrm{C}$. The cells were then kept on ice for $5 \mathrm{~min}$ until $800 \mu \mathrm{l}$ of LB medium were added. The cells were grown at $37^{\circ} \mathrm{C}$ for $40 \mathrm{~min}$ in a shaker. $50 \mu \mathrm{l}$ of the cells were plated on LB agar plates with the appropriate antibiotic and the plates were kept overnight at $37^{\circ} \mathrm{C}$ to get the transformed colonies.

\subsubsection{Protein expression and purification}

All the proteins and their variants mentioned above were expressed in E.coli BL 21 (DE3) cells. LB medium was inoculated with the appropriate transformed colony. The cells were grown overnight in a shaker at $37^{\circ} \mathrm{C}$. For expression, $10 \mathrm{ml}$ of overnight grown culture was used to inoculate $1 \mathrm{~L}$ of appropriate antibiotic containing TB medium, the cells were grown until they reached an $\mathrm{OD}_{600}$ of $\sim 0.8$. They were then induced to express the protein by the addition of IPTG (60 mg (w/v) per liter). The cells were grown for 4 hours at $37^{\circ} \mathrm{C}$ to allow expression of the recombinant protein. The cells were pelleted and resuspended in $20 \mathrm{ml}$ of resuspension buffer (20mM Tris pH-7.4, 1mM EDTA) The cells were lysed with the combination of lyzozyme treatment and sonication. To reduce the viscosity of the solution DNase was added; PMSF (200mM) was added to inhibit the activity of proteases. The lysate was centrifuged at $13,000 \mathrm{~g}$ to pellet the cell debris. The supernatant was used for the affinity purification.

All the proteins were soluble. They contained a cleavable His tag for affinity purification (The only exception is endobrevin, which contained a GST-tag). For affinity purification on $\mathrm{Ni}^{2+}$-nitrilotriacetic acid beads (Qiagen) were added; $1 \mathrm{ml}$ of beads was 
used to bind 5mg of recombinant protein. The beads were washed with excess of wash buffer $\sim 100 \mathrm{ml}$ (20mM Tris $\mathrm{pH}-7.4,500 \mathrm{mM} \mathrm{NaCl}$, and $8 \mathrm{mM}$ imidazole). The protein was eluted using three bead volumes of elution buffer (20mM Tris PH-7.4, 500mM NaCl, 400mM imidazole). The affinity purification was followed by ion exchange chromatography on an ÄKTA system (Amersham Biosciences). All the syntaxin variants, SNAP-25 and BoNT/D, complexin were purified on a Mono-Q column; the synaptobrevin and its variants were purified on a Mono-S column. Synaptobrevin (1-59) and synaptobrevin (60-96) were obtained after BoNT/D cleavage of synaptobrevin (196). Only the C-terminal fragment binds the Mono-S column and thus could be separated from the N-terminal fragment.

The tomosyn SNARE fragment bound strongly to the $\mathrm{Ni}^{2+}$-nitrilotriacetic acid matrix and was eluted using $100 \mathrm{mM}$ EDTA, $\mathrm{pH}$ 7.4, in the presence of $5 \mathrm{M}$ urea. Furthermore, as it did not bind to a Mono-Q or Mono-S column, it was run through both the columns. The flow-through was collected, and it contained a relatively pure tomosyn SNARE fragment.

Endobrevin was cloned as a GST fusion protein in pGEX-KG vector [94]. The initial affinity purification was done on GST-sepharose beads. Thrombin was added to the beads to cleave the GST-tag. The eluted endobrevin was purified by FPLC by running over a Mono-S column.

Due to the abundance of non-preferred arginine codons, the full-length amisyn was expressed at very low levels. It was conspicuous only after affinity purification with nickel beads. Full-length amisyn expression was however greatly increased in RIL cells (Stratagene); these cells contain genes in a plasmid vector that encode the non-preferred tRNAs of E.coli. However, unfavorably, almost all the highly expressed amisyn was collected in inclusion bodies. In my study only the poorly expressed soluble amisyn was used. Both amisyn and its SNARE motif were purified on a Mono-S column.

All the proteins obtained after these purification procedures were more than 95\% pure as seen from the SDS-PAGE. The proteins were dialyzed to a buffer containing 20mM Tris pH-7.4, 1mM EDTA, $1 \mathrm{mM}$ DTT and 50mM NaCl. The His-tag was always cleaved during dialysis by the addition of thrombin (10U per mg of protein). 
For the formation of a ternary complex the proteins syntaxin: SNAP25 (or its helices) and synaptobrevin (various fragments) were assembled in a 1:1:1.5 molar ratio. They were incubated at room temperature for 2 hours before purifying on a Mono-Q column to separate the unassembled monomers from the complex.

The proteins were quantified using absorption at $280 \mathrm{~nm}$, for the fragments without aromatic residues the Bradford assay was used [96].

\subsubsection{Gel Electrophoresis}

For the SDS gel electrophoresis the protocol, as suggested by Laemmli.U.K [97] was used. When better resolution, especially for the lower molecular weight fragments were needed, Schägger gels were used [98]. The non-denaturing gel electrophoresis was performed in the same way as the SDS gel electrophoresis except that SDS was omitted from all buffers.

After electrophoresis the stacking gel was discarded and the separation gel was fixed and stained for 20 min in staining solution (50\% (v/v) methanol, 10\% (v/v) acetic acid and 0.2\% (w/v) Coomassie Brilliant Blue R-250) under agitation. The gel was destained for 20 min in Destain 1 (20\% (v/v) isopropanol and 10\% (v/v) acetic acid) for 20 min and then in Destain 2 (5\% (v/v) methanol and 12. 5\% (v/v) acetic acid) until no background staining was visible. After scanning, the gel was dried in a gel dryer and preserved for further analysis.

\subsubsection{Protein labeling}

The single cysteine mutants were labeled using the sulphahydryl-reactive fluorophores Oregon Green 488 iodoacetamide, Texas Red C5 bromoacetamide or Alexa Fluor $488 \mathrm{C}_{5}$ maleimide (Molecular probes- Invitrogen). The proteins were labeled as per manufacturer's instructions. After labeling the labeled protein was separated from the dye by size exclusion chromatography on a sephadex G-25 column (Amersham biosciences). The labeling efficiency was later calculated as per the manufacturer's instructions. 


\subsubsection{Fluorescence measurements}

The fluorescence measurements were carried out in the Flurolog-3 or Fluromax 2 spectroflurometer (Jobin Yvon). Fluorescence cell with magnetic stirrer, with a path length of $10 \times 4 \mathrm{~mm}$ or with a pathlength $10 \times 10 \mathrm{~mm}$ (Hellma) was used in the experiments. All the measurements were taken in PBS $\left(150 \mathrm{mM} \mathrm{NaCl}, 20 \mathrm{mM} \mathrm{Na}_{2} \mathrm{HPO}_{4}\right.$, and $\mathrm{pH}-7.4$ ) buffer. In order to obtain reliable measurements, slit widths were adjusted accordingly so that there were at least 50,000 counts per second. All the labeled proteins were taken at a concentration of $\sim 200 \mathrm{nM}$.

Fluorescence anisotropy

The fluorescence anisotropy of a protein labeled with Texas red was measured with excitation and emission wavelengths, 590nm and 610nm respectively. For an Oregon green or Alexa 488 labeled protein, excitation and emission wavelengths $488 \mathrm{~nm}$ and 520 nm respectively was used.

The $\mathrm{G}$ factor was calculated using $\mathrm{G}=I_{\mathrm{HV}} / I_{\mathrm{HH}}$. The anisotropy $(\mathrm{r})$ was calculated using $\mathrm{r}$ $=\left(I_{\mathrm{VV}}-\mathrm{G} \times I_{\mathrm{VH}}\right) /\left(I_{\mathrm{VV}}+2 \times \mathrm{G} \times I_{\mathrm{VH}}\right)$. In all the above, $I$ refers to the fluorescence intensity, the first subscript is the direction of the exciting light and the second the direction of the emitted light.

FRET

Förster resonance energy transfer (FRET) was monitored between protein labeled with Oregon green and protein labeled with Texas red. The labeled proteins were taken at a concentration of $200 \mathrm{nM}$. The donor, Oregon green was excited at $488 \mathrm{~nm}$, the donor fluorescence and acceptor fluorescence (that of Texas red) were measured simultaneously at $520 \mathrm{~nm}$ and $610 \mathrm{~nm}$ respectively.

\subsubsection{Circular dichroism (CD) measurements}

The measurements were carried out in Jasco model J-720 spectrometer with 1mm pathlength cuvettes (Hellma). For kinetic experiments 10 x $10 \mathrm{~mm}$ path length cuvettes with stirrer was used. The kinetic and thermal denaturation measurements were carried out in PBS (150mM NaCl, 20mM Na $\left.2 \mathrm{HPO}_{4}, \mathrm{pH}-7.4\right)$ buffer. The spectra measurement was done in $10 \mathrm{mM}$ phosphate buffer $\mathrm{pH} 7.4$ buffer. Before measurement all the proteins 
were dialyzed in the appropriate buffers. The wavelength during the thermal denaturation and kinetic measurements was fixed at 222nm. For the thermal denaturation experiment the ellipticity decreases through increasing temperatures were monitored and the rate of increase was $30^{\circ} \mathrm{C}$ per hour.

\subsubsection{Crystallization and Data Collection}

Crystals of the tomosyn SNARE complex were obtained at $20{ }^{\circ} \mathrm{C}$ by the vapor diffusion technique with hanging drops. The initial protein concentration was $\sim 6.5 \mathrm{mg} / \mathrm{ml}$ in standard buffer (20mM Tris pH. 7.4, 1mM EDTA) containing $100 \mathrm{mM} \mathrm{NaCl} .1 \mu \mathrm{l}$ of protein solution was mixed with $1 \mu$ of well solution consisting of $30 \%( \pm)$-2-methyl-2, 4-pentanediol, $50 \mathrm{mM} \mathrm{CaCl}_{2}$, and $50 \mathrm{mM} \mathrm{MES,} \mathrm{pH} \mathrm{6.0.} \mathrm{The} \mathrm{crystals} \mathrm{grew} \mathrm{as} \mathrm{clustered,}$ thin plates. In 2 days, after the streak seeding of pre-equilibrated hanging drops single, thicker plates with an average size of $0.1 \times 0.4 \times 0.7 \mathrm{~mm}^{3}$ appeared. They reached their maximal size after 5 days. For data collection crystals were flash-cooled in liquid nitrogen. Diffraction data to $2.0-\AA$ resolution were collected at $100 \mathrm{~K}$ at the European Molecular Biology Laboratory BW7A synchrotron beam-line (Deutsche Electronen Synchrotron, Hamburg, Germany), using a marccd image plate scanner. Data were integrated with DENZO and scaled with SCALEPACK [99]. The Collaborative Computational Program Number 4 suite of programs [100] was employed by means of its graphical interface [101] to convert the intensities to structure factor amplitudes and for subsequent calculations.

\subsubsection{Structure Determination and Refinement}

The structure of the tomosyn SNARE complex was solved by molecular replacement with AMORE [102], using the structure of the truncated synaptic core SNARE complex [103] as a search model. Automatic model building using ARP/wARP [104] alternating with Refmac refinement was performed to obtain an initial model. Further refinement consisted of iterative cycles of interactive model building by XtalView [105] and restrained refinement by minimization of a maximum likelihood function by REFMAC [106] coupled with automatic solvent building by ARP/wARP 
[104]. Refinement and map calculation were performed with $95 \%$ of the data; $5 \%$ of randomly chosen reflections were used for the calculation of $R_{\text {free }}$.

\subsubsection{Sequence Analysis}

New homologs of tomosyn were searched for using the Blast search tool (www.ncbi.nlm.nih.gov/blast/). In addition to the sequences used for the alignments in (Fig. 25) three other tomosyn orthologs were found from the following species: Gibberella zeae, gi 42546760; Candida albicans, gi 46433698; Eremothecium gossypii, gi 45185876. The alignments were done using ClustalW (www.ebi.ac.uk/clustalw) [107]

\subsubsection{Polyclonal antibodies}

Polyclonal antibodies were generated against full-length amisyn. The rabbits were immunized with $300 \mu \mathrm{g}$ of protein emulsified in Freud's adjuvant. It was followed by booster injections that were given every three weeks for 3 to 4 months. Eventually, the antiserum was obtained from the ear veins.

\subsubsection{Preparation of amisyn antigen column}

An antigen column, with amisyn coupled to CNBr activated sepharose (coupling capacity 25-60 mg /ml) was prepared in order to isolate amisyn specific antibodies from the polyclonal antiserum.

CNBr activated sepharose was swollen for $15 \mathrm{~min}$ in $\mathrm{HCl}(1 \mathrm{mM})$ and the beads were poured into a column. The beads were washed with $1 \mathrm{mM} \mathrm{HCl}$ and neutralized with coupling buffer (0.1 $\mathrm{M} \mathrm{NaHCO}_{3}, 0.5 \mathrm{M} \mathrm{NaCl}, \mathrm{pH}$ 8.3) before the protein was loaded. The protein was bound to $\mathrm{CNBr}$ activated sepharose by incubating at $4{ }^{\circ} \mathrm{C}$ over night. After incubation the flow through was collected and checked for coupling efficiency by Bradford protein assay. The column was further incubated with blocking agent $(1.5 \mathrm{mM}$ Tris $\mathrm{pH}$ 8.9) on a rotating wheel for $2 \mathrm{~h}$ at $\mathrm{RT}$ to block unspecific binding sites. The beads were then alternately washed 3 times with coupling buffer and then acetate buffer (0.1 $\mathrm{M}$ acetate, $0.5 \mathrm{M} \mathrm{NaCl}, \mathrm{pH}$ 4.0). The column was stored in PBS containing $0.1 \%$ $\mathrm{NaN}_{3}$. 


\subsubsection{Affinity purification of Amisyn antibodies}

The amisyn antigen-column was used to isolate amisyn specific antibodies. Before applying the polyclonal antiserum on the column, the column was washed with 8 column volumes TBS-0.1\% Tween (pH 7.3), $0.1 \mathrm{M}$ Glycine (elution buffer, $\mathrm{pH} 2.2$ ) and TBS-0.1\% Tween. The column was incubated with antiserum at $4{ }^{\circ} \mathrm{C}$ over night. After the incubation the flow through was collected. Before elution, the column was washed again with TBS-0.1\% Tween ( $\mathrm{pH} 7.3$ ) and TBS ( $\mathrm{pH}$ 7.3). Elution was done using $0.1 \mathrm{M}$ glycine and the eluate was immediately neutralized with $1.5 \mathrm{M}$ Tris (pH 7.3). Native and recombinant amisyn was blotted onto nitrocellulose membrane and it was used to check if the eluate contained antibodies specific for amisyn. The ECL method was used to detect the presence of bound antibodies.

\subsubsection{Preparation of synaptic vesicles}

The protocol as suggested in [108-110] was used to prepare synaptic vesicles. Briefly, 20 rat cerebral cortices were homogenized in $150 \mathrm{ml}$ of sucrose buffer $(0.32 \mathrm{M}$ sucrose, 5mM HEPES-NaOH pH 7.3, 0.1mM PMSF, $2 \mu \mathrm{g} / \mathrm{ml}$ pepstatin A). The homogenate $(\mathrm{H})$ was spun for 10 min at $800 \mathrm{~g}$; yielding a pellet $\left(\mathrm{P}_{1}\right)$. The supernatant was centrifuged at $9200 \mathrm{~g}$ for 15 min. The pellet was washed once in sucrose buffer and a crude synaptosomal pellet $\left(\mathrm{P}_{2}\right)$ was obtained. (Synaptosomes are artificial structures; it is a membranous sac that contains the nerve terminal. They are produced after selective centrifugation of the nerve tissue homogenate). The supernatant was centrifuged at $165,000 \mathrm{~g}$ for $1 \mathrm{~h}$, yielding a pellet $\mathrm{P}_{3}$ and supernatant $\mathrm{S}_{3}$. $\mathrm{P}_{2}$ was resuspended in $12 \mathrm{ml}$ of sucrose buffer and it was lysed and homogenized by the addition of 9 volumes of distilled water. HEPES/NaOH buffer ( $\mathrm{pH} 7.4$ ) was added to a final concentration of $10 \mathrm{mM}$. After 30 min incubation, the fraction was spun at 25,000g for $20 \mathrm{~min}$ (pellet $\mathrm{LP}_{1}$ ), and the supernatant was further centrifuged for $2 \mathrm{~h}$ at 165,000g, yielding a crude synaptic vesicle pellet $\left(\mathrm{LP}_{2}\right)$ and a supernatant $\left(\mathrm{LS}_{2}\right)$. Vesicles were further purified on a continuous sucrose density gradient yielding the fraction SGV. To evaluate the purity of synaptic vesicles we did electron microscopy in collaboration with Dr. Dietmar Riedel from our 
institute. Furthermore, western blots were done to check for the enrichment of synaptophysin. (Synaptophysin is a membrane protein on the synaptic vesicles and we could visualize a several fold enrichment of synaptophysin during the purification procedure).

\subsubsection{Western Blot}

In a western blot the proteins after gel electrophoresis are transferred to a nitrocellulose membrane in order to detect the protein of our interest using a specific antibody. I used a semi-dry western blot apparatus for the purpose. The gel and the membrane were sandwiched between blotting papers (GB003 from Schleicher \& Schuell). Before blotting, the blotting papers and the membrane were soaked in transfer buffer (25mM Tris/HCl pH-8.3, 193mM glycine, 20\% (v/v) methanol, 0.1\% (w/v) SDS). The transfer was carried out at 50mA constant current for 1 hour. Prestained marker was run on the gel and the efficiency of transfer was directly evaluated.

After transfer, the membrane was either stored at $4^{\circ} \mathrm{C}$ or it was directly proceeded with blocking. To the membrane, blocking solution (5 \%( w/v) dry milk powder in PBS) was added and kept in a shaker for $30 \mathrm{~min}$. The membrane was then washed three times, 10 min each, using the washing solution $(0.1 \%$ Tween-20, 200mM Tris- $\mathrm{HCl} \mathrm{pH} 7.7$, $0.15 \mathrm{M} \mathrm{NaCl}$ ). After washing, the primary antibody, which was diluted 1000 times in blocking solution, was added to the membrane. It was either left overnight in a shaker at $4^{\circ} \mathrm{C}$ or kept in a shaker for 1 hour at room temperature. To remove unspecifically bound antibodies the membrane was washed three times, $10 \mathrm{~min}$ each, with washing solution. After washing, the secondary antibody that was diluted 2000 times in blocking solution was added. The membrane was kept in a shaker for $30 \mathrm{~min}$ at room temperature. The membrane was washed three times, 10 min each, with washing solution to remove the unbound secondary antibody.

The secondary antibody was coupled to horseradish peroxidase so that it emits luminescence after the addition of chemiluminiscence reagent (Perkin Elmer). The luminescence was detected using the luminescent image analyzer. 


\subsubsection{Preparation of proteoliposomes}

Lipids (Avanti, Alabaster, AL) were mixed in chloroform to yield (molar ratios): phosphatidylcholine (5),phosphatidylethanolamine (2),phosphatidylserine (1),phosphatid ylinositol (1) and cholesterol (1). After drying, they were resuspended in $20 \mathrm{mM}$ HEPES/KOH pH $7.4100 \mathrm{mM} \mathrm{KCl,} 5 \mathrm{mM}$ DTT, 5\% (w/v) sodium cholate at a total lipid concentration of $13.5 \mathrm{mM}$. SNARE-proteins in $20 \mathrm{mM}$ sodium cholate were added (lipid to protein ratio of 200:1 $\mathrm{n} / \mathrm{n}$ ); followed by size-exclusion chromatography on a PC 3.2/10 Fast Desalting column (GE Healthcare) equilibrated in $20 \mathrm{mM}$ HEPES/KOH pH 7.4, $150 \mathrm{mM} \mathrm{KCl}, 1 \mathrm{mM}$ DTT. For the preparation of NBD/Rhodamine labeled liposomes $1.5 \%$ (n/n) 1,2-Dioleyl-sn-Glycero-3-Phosphoethanolamine-N-Lissamine Rhodamine B Sulfonyl, $1.5 \%$ (n/n) 1,2-Dioleyl-sn-Glycero-3-Phosphoethanolamine-N-(7-nitro-2-1,3benzoxadiazol-4-yl) and $17 \%$ (n/n) phosphatidylethanolamine were used instead of $20 \%$ $(\mathrm{n} / \mathrm{n})$ phosphatidylethanolamine.

\subsubsection{Liposome fusion assay}

Liposome fusion reactions were performed at $30^{\circ} \mathrm{C}$. For reactions $15 \mu \mathrm{l}$ of labeled and unlabelled liposomes were mixed in a total volume of $1.2 \mathrm{ml}$ resulting in final protein concentrations of approx. $200 \mathrm{nM}$ for both liposome populations. Fluorescence dequenching was measured using $460 \mathrm{~nm}$ for excitation and $538 \mathrm{~nm}$ for emission. Fluorescence intensities were normalized to the initial fluorescence intensity. 


\section{Results}

\subsection{A helix replacement reaction sheds new light on SNARE mediated membrane fusion}

The three neuronal SNAREs syntaxin 1a, SNAP-25A and synaptobrevin 2 interact and form a highly stable coiled-coil bundle [25], which is referred to as the ternary complex. The ternary complex, once formed, does not dissociate in a biologically relevant timescale [42] and it is not in an equilibrium with unassembled monomers.

\subsubsection{Replacement of the synaptobrevin helix}

Several stable ternary complexes (Tc), with different shortened versions of synaptobrevin were purified. All the ternary complexes contained the SNARE motif of syntaxin (H3 domain residues 180-262) and full length SNAP-25A. Many of these ternary complexes are 'SDS resistant' i.e. the complexes do not dissociate into monomers (unless heated) under the conditions of SDS-PAGE. To the ternary complexes full length soluble synaptobrevin (residues 1-96) was added. When it was mixed with ternary complexes Tc (42-96), Tc (61-96), Tc (1-70) and Tc (1-65) surprisingly, a complex equivalent to the size of Tc (1-96) appeared (Fig. 9A). (The residue numbers of synaptobrevin in the ternary complex is indicated within brackets). It suggests that the shortened synaptobrevins in these ternary complexes gets replaced by the externally added soluble synaptobrevin (Fig. 9A). In all the other complexes no replacement was seen (Fig. 9A).

In another approach, full length soluble synaptobrevin (residues 1-96) labeled with Texas Red at position 28 (Sb*) (Fig. 9B) was added to various ternary complexes. After separation on a SDS-PAGE gel the position of labeled synaptobrevin was monitored using a luminescent image analyzer. A higher molecular weight 'SDS resistant' band corresponding to the size of a ternary complex (arrow head) was formed in Tc (42-96), Tc (61-96), Tc (1-70) and Tc (1-65) (Fig. 9B). In line with the above results (Fig. 9A), it demonstrates that the labeled synaptobrevin formed a ternary complex by replacing the shortened synaptobrevins from their respective complexes. It 
also confirmed that replacement did not occur in Tc (1-96). The result was independent of labeling position and the nature of the dye. (data not shown).

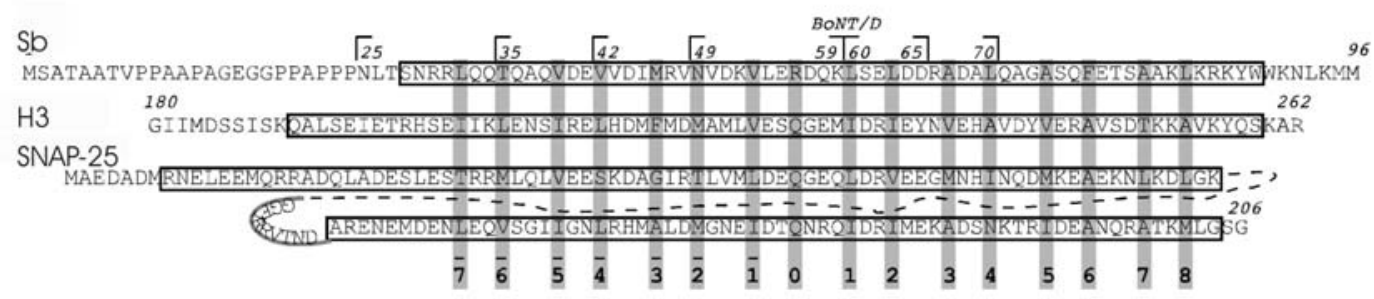

\section{Figure 8 Alignment of SNARE motifs}

Sequence of the SNARE core complex, syntaxin (H3), SNAP-25 and synaptobrevin (Sb) are aligned. Positions of the heptad repeat layers $(-7$ to +8$)$ are indicated in gray. The structure of the neuronal core SNARE complex [25] is boxed. The residue numbers of synaptobrevin fragments used in this study are shown.

\section{A}

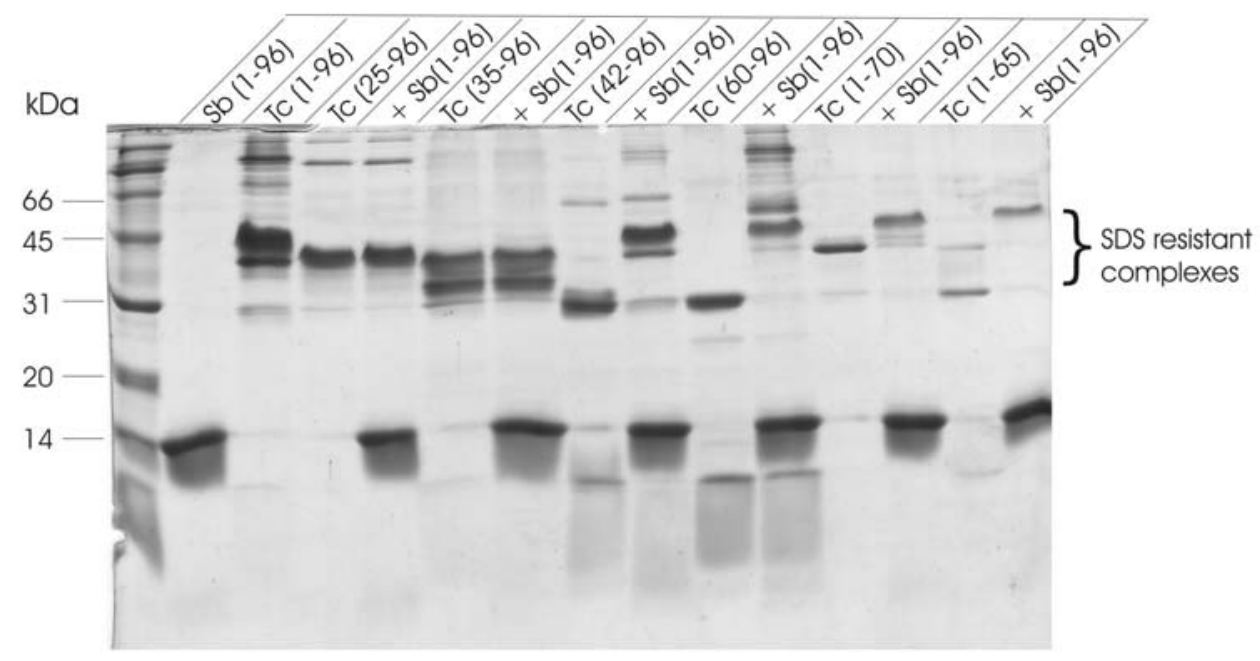




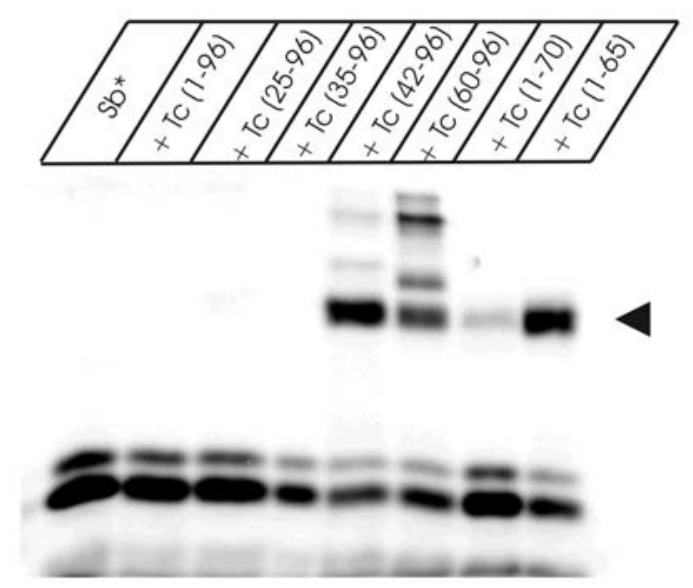

Figure 9 Replacement of the synaptobrevin helix

(A) Various purified ternary SNARE complexes (Tc $\sim 2 \mu \mathrm{g}$ each), containing a different truncated synaptobrevin variant, were mixed with Syb1-96 $(\approx 2 \mu \mathrm{g})$ for two hours at room temperature and afterwards separated by SDS-PAGE without prior heating of the samples. The fragment length of the synaptobrevin in the ternary complex is indicated within brackets. In the first two lanes Syb1-96 and the purified ternary SNARE complex containing Sb (1-96) respectively were loaded. New SDS-resistant complexes containing the added Sb (1-96) formed only for the complexes Tc (42-96), Tc (60-96), Tc (1-70), and Tc (1-65). Note that some of the ternary complexes containing synaptobrevin fragments are also SDS-resistant, sometimes only partially, but then usually run at lower molecular mass than Tc (1-96). (B) As above, the various SNARE complexes were incubated with Texas Red labeled Syb1-96 (Sb*; $\approx 1 \mu \mathrm{g}$ ). SDS-resistant complexes containing fluorescent synaptobrevin (arrow head) formed only in the complexes Tc (42-96), Tc (60-96), Tc (1-70), and Tc (1-65).

\subsubsection{Fast binding of synaptobrevin to $\Delta \mathbf{N}$ ternary complexes}

Fluorescence anisotropy measurements were used to gain insights into the speed of the replacement reaction. Synaptobrevin (residues 1-96) labeled at position 28 with Texas Red was added to various short synaptobrevin containing ternary complexes (Tc) and the change in fluorescence anisotropy was monitored (Fig. 10).

A rapid anisotropy increase was seen after the addition of the ternary complexes Tc (42-96), Tc (49-96) and Tc (60-96) to the labeled synaptobrevin (The numbers inside the brackets represent amino acid residues of synaptobrevin in the ternary complex). A relatively slow increase was observed for Tc (1-65) and Tc (1-59). Anisotropy increase suggests association of labeled synaptobrevin with the ternary complex. 
The residues 28-89 in synaptobrevin form the SNARE motif [25], therefore the result indicates that the anisotropy change was faster for ternary complexes containing the $\mathrm{N}$-terminally shortened synaptobrevin ( $\Delta \mathrm{N}$ ternary complexes) than for the ternary complexes with $\mathrm{C}$-terminally shortened synaptobrevin ( $\Delta \mathrm{C}$ ternary complexes). Rapid anisotropy increase as observed after the addition of Tc (42-96), Tc (49-96) and Tc (6096) was due to rapid binding of the labeled synaptobrevin to these complexes. Anisotropy change was observed only in complexes, with the exception of Tc (1-70), were the replacement occurred (Fig. 9A and B). In Tc (1-70) the change was too slow to be apparent at the measured concentrations and after overnight incubation anisotropy increase was observed (data not shown).

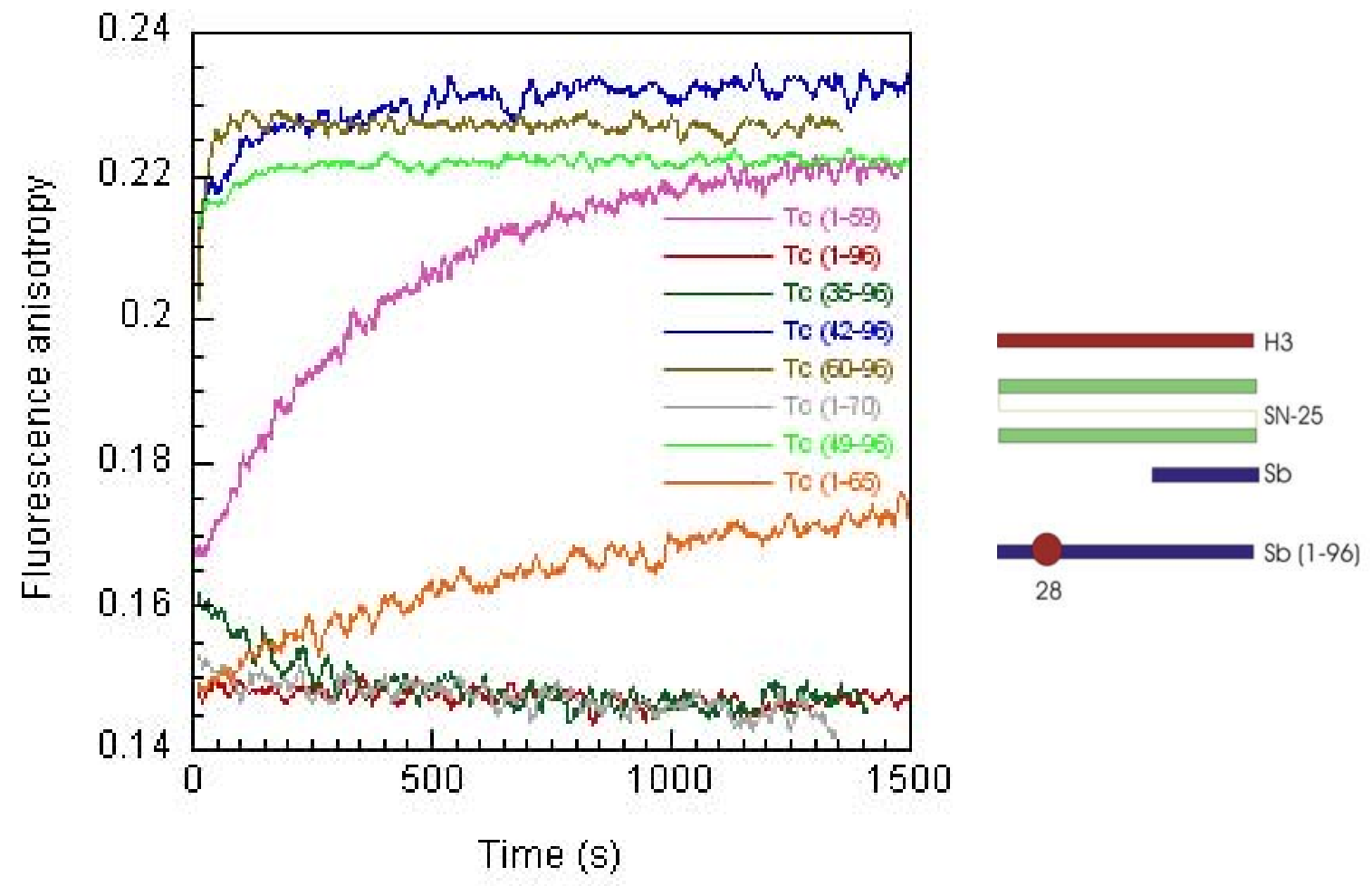

Figure 10 Fast binding of synaptobrevin to $\Delta \mathbf{N}$ ternary complexes

Anisotropy change after the addition of various shortened synaptobrevin containing ternary complexes (Tc, $1 \mu \mathrm{M})$ to synaptobrevin labeled at position 28 with Texas Red (200nM). A rapid increase in fluorescence anisotropy was observed after the addition of Tc (42-96), Tc (4996) and Tc (60-96) and a relatively slow anisotropy increase was seen after the addition of Tc (165) and Tc (1-59). The numbers inside the brackets represent amino acid residues of synaptobrevin in the ternary complex. After an overnight incubation, anisotropy increase was 
Results

observed in Tc (1-70). In all the other complexes no change was observed. The cartoon shows the design of the experiment; the ternary complexes used in the experiment contained the $\mathrm{H} 3$ domain of syntaxin, SNAP-25 and synaptobrevin (various fragments).

\subsubsection{Shortened synaptobrevin replacement kinetics}

Anisotropy measurements (Fig. 10) gave information about how fast the soluble synaptobrevin associated with various shortened synaptobrevin complexes. In order to monitor the replacement of shortened synaptobrevin from the ternary complex, FRET (Fluorescence resonance energy transfer) between syntaxin (residues 180-262 labeled at position 225 with Texas Red) and synaptobrevin (residues 42-96 labeled at position 61 with Oregon Green) was used (Fig. 11). The two labeled proteins were mixed together and, after the excitation of the donor, the donor and acceptor fluorescence were measured. Addition of SNAP-25 to the labeled proteins led to the formation of a 'FRETactive' ternary complex. The donor and acceptor fluorescence were measured again. Due to FRET, the donor fluorescence decreased and the acceptor fluorescence increased (in Fig. 11 only the donor fluorescence is shown).

Sb (1-96) was added to the 'FRET-active' complex and the change in donor and acceptor fluorescence was monitored. The donor fluorescence increased after the addition of Sb (1-96). Similarly, addition of Sb (25-96) and endobrevin (another member of the synaptobrevin family involved in endosomal trafficking) also increased the donor fluorescence. The increase in donor fluorescence indicates replacement of the labeled synaptobrevin (residues 42-96, 61 Texas Red) in the ternary complex by these unlabeled synaptobrevin fragments. The rate of replacement was similar for Sb (1-96) and endobrevin, and was slower with Sb (25-96). Similar kinetics were observed when the replacement reaction was monitored using fluorescence anisotropy (data not shown). The replacement reaction was very slow with Sb (1-70), and the other synaptobrevin fragments viz Sb (35-96) and Sb (42-96) did not change the donor fluorescence suggesting that they were unable to replace the Sb (42-96) from the ternary complex.

The Sb (42-96) displacement rate was not rapid therefore the rapid anisotropy change (Fig. 10) that was observed after the addition of Sb (1-96) to Tc (42-96) represented only the binding of synaptobrevin to Tc (42-96). The SNARE motif of synaptobrevin begins at residue 28; Sb (35-96) did not replace Sb (42-96) from the 
complex. It is very likely that the N-terminal deletion inside the SNARE motif hindered Sb (35-96) from efficient binding to Tc (42-96), and without efficient binding there could not be efficient replacement. Additionally, the inability of Sb (1-70) to efficiently replace short synaptobrevin from the ternary complex suggested that C-terminal residues in the externally added synaptobrevin are important for replacement.

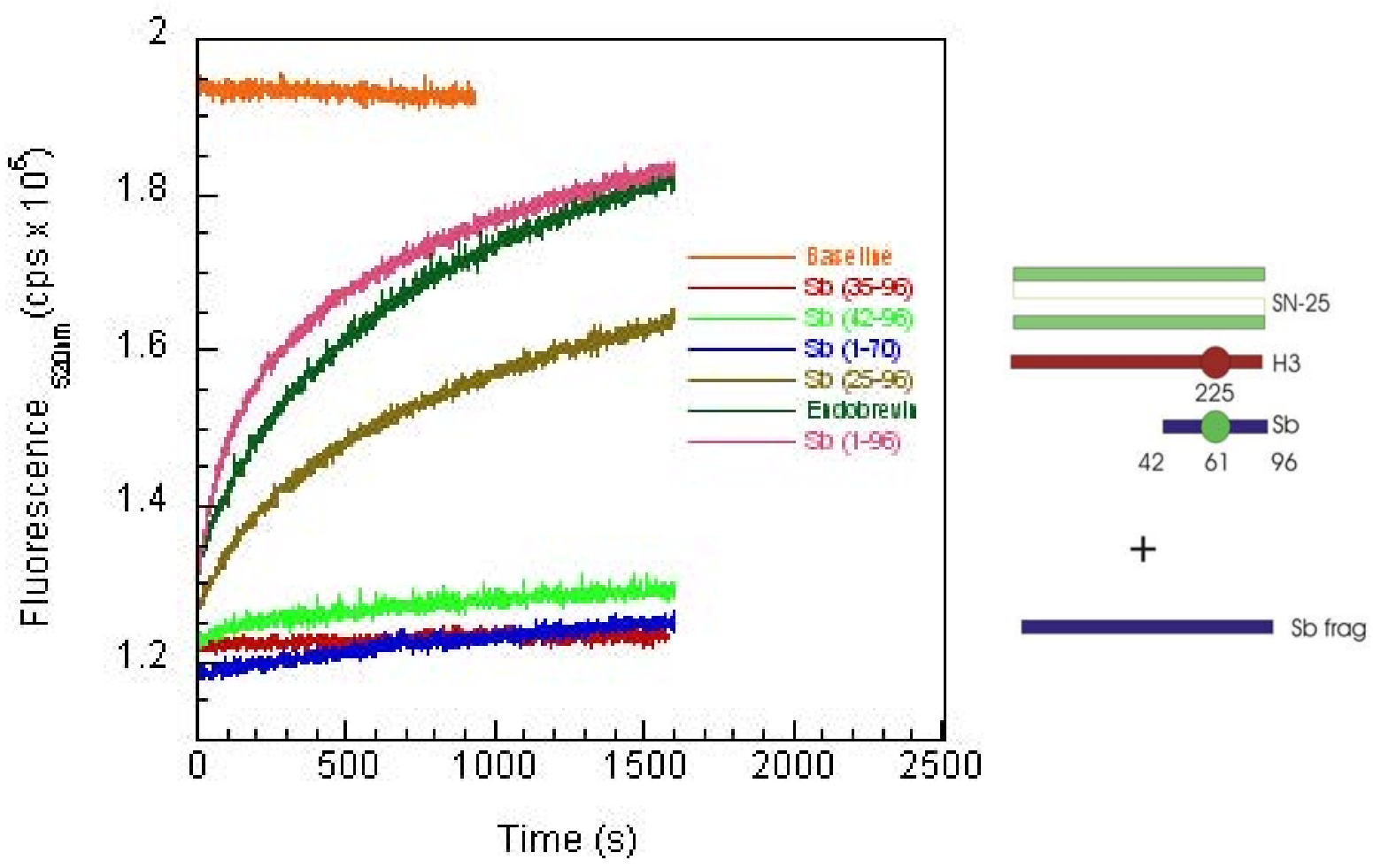

Figure 11 Shortened synaptobrevin replacement kinetics

Donor fluorescence measured in the presence of syntaxin (residues 180-262) labeled at position 225 with Texas Red (200nM) and synaptobrevin (residues 42-96) labeled at position 61 with Oregon Green (200nM) after excitation of Oregon green at 488nm (Baseline). Addition of SNAP$25(1 \mu \mathrm{M})$ caused a considerable decrease in donor fluorescence due to FRET. Various unlabelled synaptobrevin fragments were added; the donor fluorescence reverted in the case of Sb (1-96), Sb (25-96) and Endobrevin, due to replacement of labeled synaptobrevin (42-96) from the complex. Sb (1-70) replaced very slowly and no significant change was observed with the other synaptobrevin fragments. The cartoon shows the experimental design.

\subsubsection{Shortening synaptobrevin enhances the replacement rate}

The following experiment was done to investigate whether the replacement rate was enhanced by shortening the length of synaptobrevin in the ternary complex. Synaptobrevin (residues 49-96) with a cysteine at residue number 79 was labeled with 
Alexa 488. A ternary complex containing this labeled synaptobrevin was assembled and purified. The fluorescence anisotropy of this ternary complex was measured. To this ternary complex, Sb (1-96) was added, after its addition a decrease in anisotropy was observed (Fig. 12). Similarly several other synaptobrevin fragments with the exception of Sb (35-96) and Sb (42-96) caused a decrease in fluorescence anisotropy (Fig. 12). A decrease in fluorescence anisotropy indicates replacement of the labeled synaptobrevin from the complex. Clearly, the replacement rate was greatly enhanced compared to the replacement rate in Tc (42-96) (Fig. 11). Furthermore, the synaptobrevin fragments Sb (1-65) and Sb (1-70) efficiently replaced Sb (49-96) (Fig.12) but were unable to replace $\mathrm{Sb}$ (42-96) from the complex (Fig.11).

This experiment also showed that the replacement rate was decreased by further shortening the C-terminus of the externally added synaptobrevin; for example Sb (1-96) replaced more efficiently than Sb (1-65).

The externally added Sb (35-96) and, Sb (42-96) did not effectuate replacement (Fig. 12). As discussed before (section 3.1.3), this is probably due to inefficient binding.

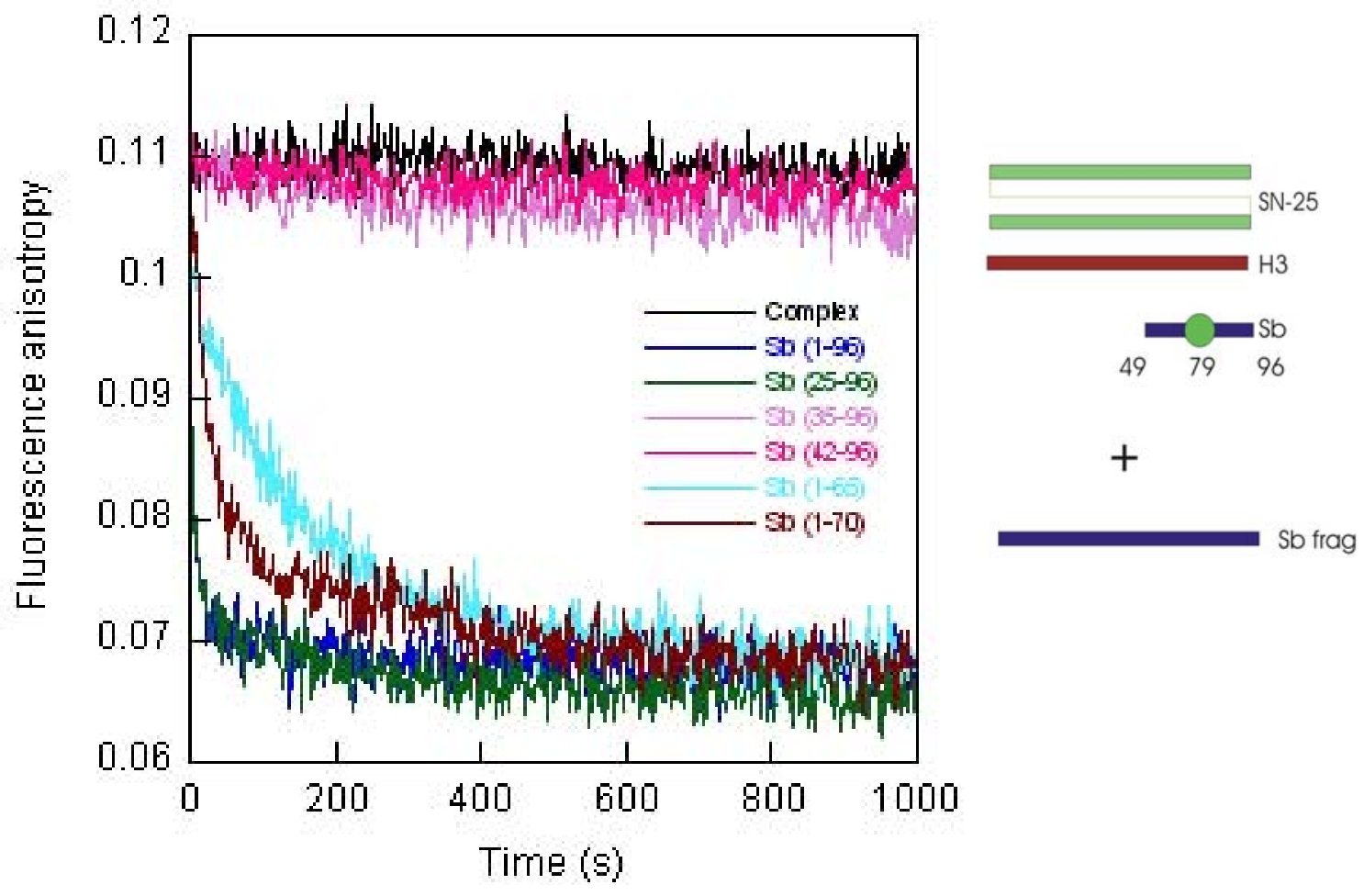


Results

Figure 12 Displacement of Sb (49-96) monitored by fluorescence anisotropy

A ternary complex containing Alexa488 labeled Sb (49-96) was purified. Various synaptobrevin fragments $(1 \mu \mathrm{M})$ were added to this complex $(200 \mathrm{nM})$. Replacement of labeled synaptobrevin led to a decrease in fluorescence anisotropy. The N-terminally shortened Sb (35-96) and Sb (42-96) were not able to replace the labeled synaptobrevin. C-terminally shortened Sb fragments were less efficient than Sb (1-96) in replacing the labeled synaptobrevin. The cartoon shows the experimental design.

\subsubsection{An open binding site is required for rapid binding}

The $\Delta \mathrm{N}$ ternary complex lacks the $\mathrm{N}$-terminal part of the synaptobrevin SNARE motif. An externally added synaptobrevin binds rapidly to $\Delta \mathrm{N}$ ternary complexes (Fig. 10). It is very likely that the $\mathrm{N}$-terminal region in the $\Delta \mathrm{N}$ ternary complex, which contains only syntaxin and SNAP-25, offers an appropriate binding site to facilitate rapid binding. In the next set of experiments I test whether this $\mathrm{N}$-terminal region is really required for rapid binding and whether binding could be abolished by blocking this site.

The fluorescence anisotropy of Texas Red was measured after coupling it to the residue 28 on Sb (1-96) fragment (Sb*28). As described before (Fig. 10) the fluorescence anisotropy of Sb*28 increased rapidly upon binding to Tc (60-96). In the presence of $\mathrm{Sb}$ (1-59) the binding was slower (Fig. 13) indicating that Sb (1-59) and Sb*28 compete for the same binding site. Furthermore, no anisotropy change was observed after preincubation of Tc (60-96) with unlabelled Sb (1-96) (Fig. 13), in this experiment Sb (1-96) replaces Sb (60-96) from the complex and occludes the binding site. Taken together, these experiments suggest that an open-binding site is required for rapid synaptobrevin binding. 


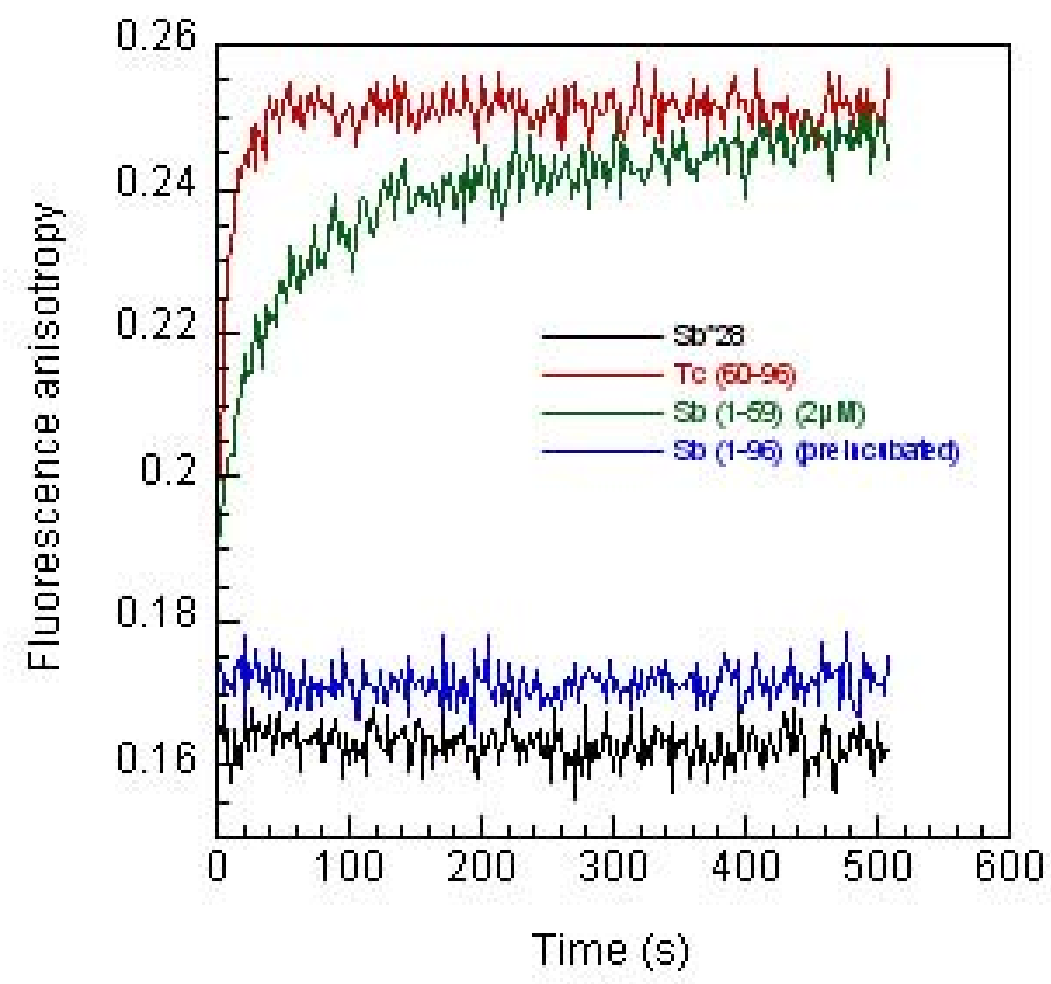

Figure 13 An open-binding site is required for rapid binding

Fluorescence anisotropy of synaptobrevin (residues 1-96) (200nM) labeled at position 28 with Texas red (Sb*28). Anisotropy increased rapidly after the addition of Tc (60-96) $(1 \mu \mathrm{M})$. The rate of anisotropy increase decreased in the presence of Sb (1-59) $(2 \mu \mathrm{M})$. After preincubation of Tc (60-96) with unlabelled Sb (1-96) no change in anisotropy was observed.

\subsection{6 $\alpha$ - helical structure in the $\mathbf{N}$-terminal half of the $\Delta \mathbf{N}$ ternary complex}

Full-length soluble synaptobrevin, Sb (1-96), bound rapidly to $\Delta \mathrm{N}$ ternary complexes and the binding rate was relatively slow with $\Delta \mathrm{C}$ ternary complexes (Fig. 10). It is probable that $\Delta \mathrm{N}$ ternary complexes offer a 'readily-available' binding site so that full-length soluble synaptobrevin binds rapidly to these complexes. Furthermore in $\Delta \mathrm{N}$ ternary complexes the synaptobrevin binding occurred at the $\mathrm{N}$ - terminal region, which contained syntaxin and SNAP-25 helices (Fig.13). Here I test whether this N-terminal region is structured to facilitate rapid synaptobrevin binding. SNARE complexes are $\alpha$ helical and show the characteristic spectra with minima at 208 and 222nm [30]. The $\Delta \mathrm{N}$ and $\Delta \mathrm{C}$ ternary complexes also show the characteristic $\alpha$ - helical spectra. The CD spectrum of the $\Delta \mathrm{N}$ ternary complex containing Sb (60-96) fragment was taken before and after the addition of $\mathrm{Sb}$ (1-59). The addition of $\mathrm{Sb}$ (1-59) to the $\Delta \mathrm{N}$ ternary complex 
Tc (60-96), caused no further change in ellipticity (Fig.14A). The SNAREs are unstructured as monomers and become $\alpha$ - helical upon interaction with their complementary SNAREs. The gain in $\alpha$ - helical content is reflected by an increase in the ellipticity in CD spectra [30]. Ellipticity did not change after the addition of Sb (1-59) to Tc (60-96), which suggests that the $\mathrm{N}$-terminal region of $\Delta \mathrm{N}$ ternary complex was already $\alpha$ - helical. It is very likely that because of a structured binding site synaptobrevin is able to bind rapidly to $\Delta \mathrm{N}$ ternary complexes.

Interestingly, in contrast, addition of $\mathrm{Sb}$ (60-96) to the $\Delta \mathrm{C}$ ternary complex Tc (159) caused a pronounced increase in ellipticity (Fig. 14B). It suggests that the C-terminal part of the $\Delta \mathrm{C}$ complex undergoes a structural change after binding of $\mathrm{Sb}$ (60-96). Therefore it is likely that the slow binding of synaptobrevin to $\Delta C$ complexes (Fig. 10) is due to the lack of a 'readily- available' binding site.

A

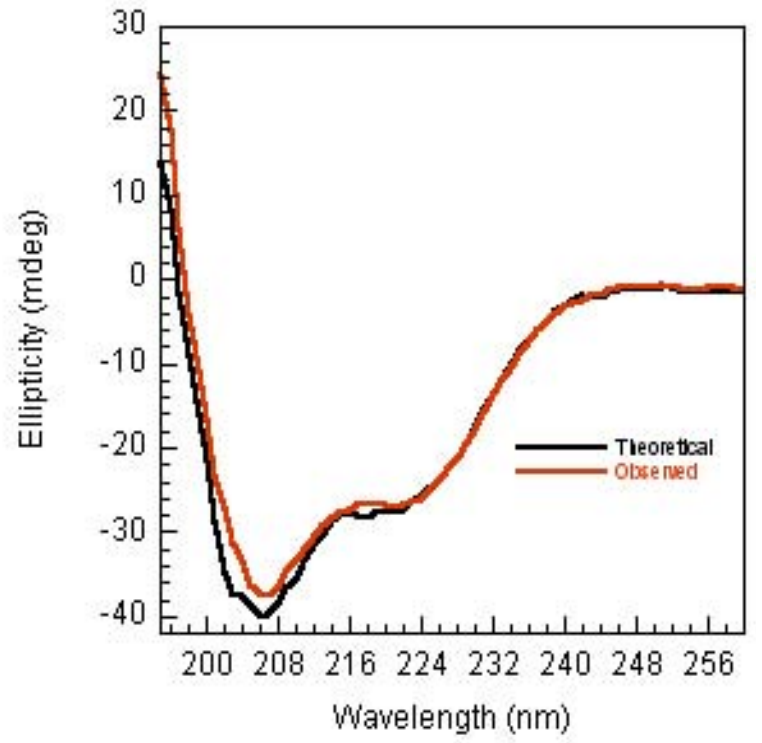

B

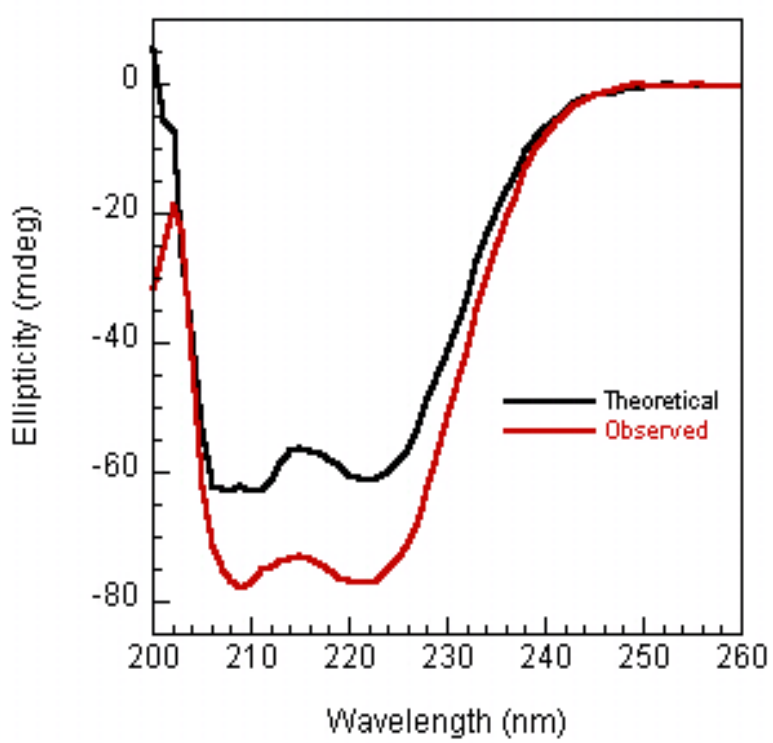

Figure $14 \alpha$-helical structure in the $\mathbf{N}$-terminal half of the $\Delta \mathbf{N}$ ternary complex (A) Theoretically combined spectra of Tc $(60-96,6 \mu \mathrm{M})$ and Sb $(1-59,12 \mu \mathrm{M})$ (Theoretical). The spectra observed after the addition of Sb (1-59) to Tc (60-96) (Observed). (B) Similarly, theoretically combined spectra of Tc (1-59) and Sb (60-96) (Theoretical). The spectra observed after the addition of Sb (60-96) to Tc (1-59) (observed). 


\subsubsection{A SNARE complex containing two synaptobrevin halves unfolds in two steps}

In the following experiment I monitor the stability of ternary complex containing either the N-terminal half or the C-terminal half of the synaptobrevin SNARE motif. The $\Delta \mathrm{N}$ ternary complex Tc (60-96) and the $\Delta \mathrm{C}$ ternary complex Tc (1-59) were used. Both complexes exhibited characteristic $\alpha$-helical CD spectra with minima at 208 and 222nm. The thermal unfolding of the complexes was monitored by CD spectroscopy, the wavelength was set at 222nm and the ellipticity decrease over increasing temperature was monitored. The $\Delta \mathrm{N}$ complex, Tc (60-96) unfolded cooperatively at about $44^{\circ} \mathrm{C}$, whereas the $\Delta \mathrm{C}$ complex, Tc (1-59) was more stable and unfolded cooperatively at about $66^{\circ} \mathrm{C}$ (Fig. 15).

Two step melting behavior was observed after the addition of Sb (60-96) to Tc (159. It indicates that after Sb (60-96) addition a new ternary complex containing both halves of the synaptobrevin SNARE motifs was formed. Similarly, addition of Sb (1-59) to Tc (60-96) also resulted in a similar two step melt (data not shown). The addition of Sb (1-59) greatly increased the thermal stability of Tc (60-96); the unfolding temperature of the second step was about $66^{\circ} \mathrm{C}$. Such a two step melting behavior suggests that the two halves of the SNARE complex unfold independently and the first unfolding step corresponds to the unfolding of the C-terminal half of the complex and second to the unfolding of the N-terminal half of the complex. 


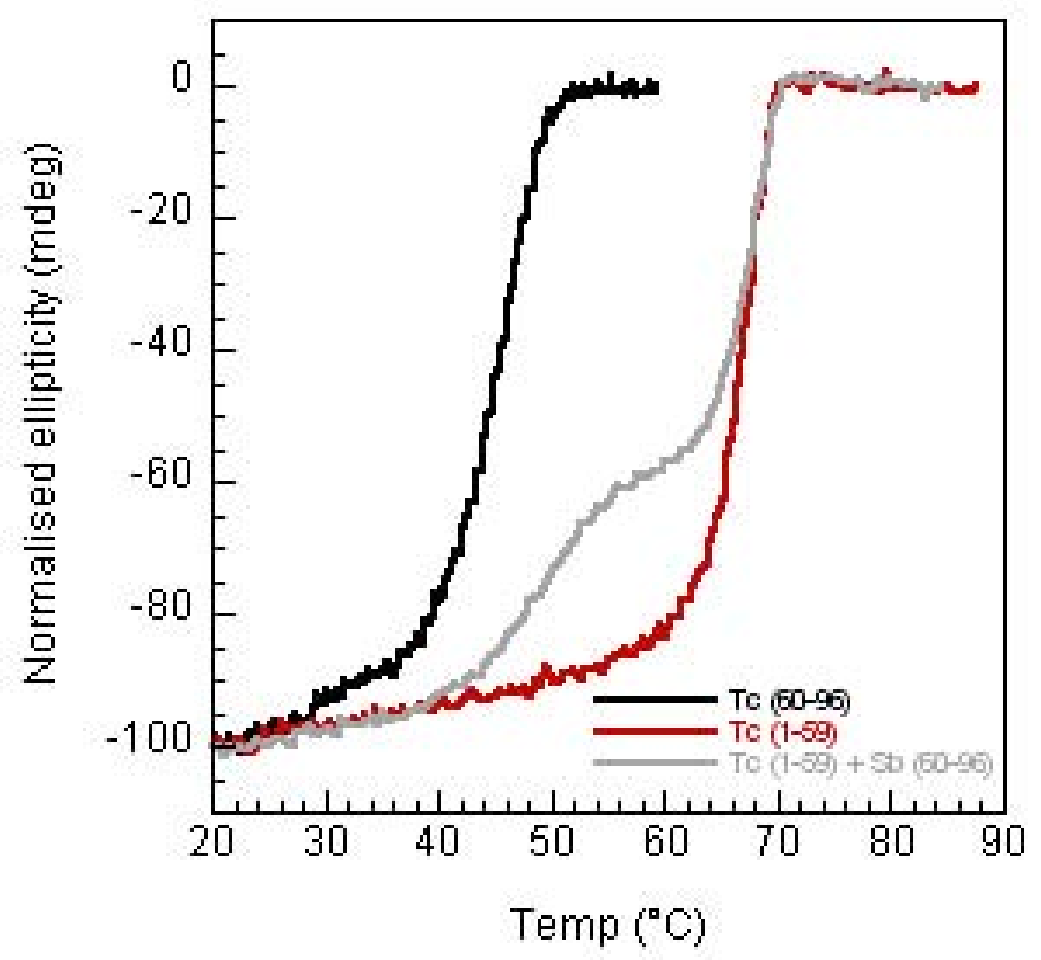

Figure 15 A SNARE complex containing the two synaptobrevin halves unfolds in two steps Thermal unfolding of Tc (60-96) $(\Delta \mathrm{N})$ and Tc (1-59) ( $\Delta \mathrm{C})$ complexes. The ternary complex Tc (60-96) unfolded cooperatively at about $44^{\circ} \mathrm{C}$, whereas Tc (1-59) unfolded cooperatively at about $66^{\circ} \mathrm{C}$. Addition of Sb (60-96) to Tc (1-59) resulted in a two step melt. Similar result was observed when Sb (1-59) was added to Tc (60-96) (data not shown).

\subsubsection{Enhancement of liposome fusion}

After reconstitution, the neuronal SNAREs are capable of fusing liposomes [65], however the liposome fusion occurs only in the order of minutes. It is a long-standing controversy in the SNARE field that neuronal SNARE mediated liposome fusion fail to mimic the speed of physiological synaptic vesicle fusion. The liposome fusion experiments monitor fusion between syntaxin/SNAP-25 (acceptor) and synaptobrevin (donor) liposomes. We reasoned that liposome fusion might be slow because fusion is monitored under the conditions that favor the 'off-pathway' reaction (Fig. 6). This means that the acceptor liposomes contain syntaxin and SNAP-25 in 2:1 stoichiometry. Synaptobrevin cannot actively replace the second syntaxin and the second syntaxin needs to leave the 1:1 dimer in order to enable synaptobrevin to bind. In solution, after the dissociation of the second syntaxin, both synaptobrevin and the second syntaxin equally 
compete for the binding site i.e. 1:1 dimer. However in acceptor liposomes it is likely that the second syntaxin has a competitive advantage as it is in the same membrane as the $1: 1$ dimer. In other words, the liposome fusion is probably slow as the binding site of synaptobrevin is blocked.

The $\Delta \mathrm{N}$ ternary complex has certain advantages over 2:1 complex. It prevents the off-pathway (i.e. the second syntaxin binding) in the neuronal SNARE assembly and it offers a well-structured site that enables fast binding of synaptobrevin (Fig. 14A). Furthermore, the synaptobrevin fragment in the complex could be replaced (Fig. 11, 12). Thus possibly the $\Delta \mathrm{N}$ ternary complex might offer a faster fusion pathway.

To test this idea, liposome fusion was monitored using $\Delta \mathrm{N}$ ternary complex. The experiment was carried out in collaboration with my colleague Alexander Stein. We formed a $\Delta \mathrm{N}$ ternary complex, which contains the SNARE motif and transmembrane domain of syntaxin (residues 183-288), SNAP-25 and Sb (49-96). This $\Delta \mathrm{N}$ ternary complex was purified and incorporated into a population of liposomes (the acceptor liposomes) and the full-length synaptobrevin, Sb (1-116) was incorporated into another population of liposomes (donor liposomes). The fusion was monitored using a dequenching assay [65]. The donor liposomes contain quenched populations of two fluorophores viz NBD and Rhodamine coupled to lipids. Fusion with acceptor liposomes increases the surface area and therefore the average distance between the fluorophores, resulting in an increase in donor (NBD) fluorescence.

When we compared the fusion between $\Delta \mathrm{N}$ ternary complex and the 2:1 complex we found that $\Delta \mathrm{N}$ ternary complex greatly enhanced the fusion rate (Fig. 16). This suggests that the liposome fusion is time-consuming because of the 'off-pathway'. To allow fusion the synaptobrevin fragment in the $\Delta \mathrm{N}$ ternary complex probably needs to be displaced by the synaptobrevin on donor liposomes. Therefore this enhanced fusion is still an underestimate as it includes the Sb (49-96) replacement kinetics. Replacement rate was enhanced by decreasing the length of synaptobrevin in the ternary complex (Fig. 12). Therefore it seems possible that fusion rate could be further enhanced using $\Delta \mathrm{N}$ ternary complex with even shorter synaptobrevin fragments. Unfortunately we could not 
form a stable complex with syntaxin (183-288), SNAP-25 and the next available short synaptobrevin, Sb (60-96).

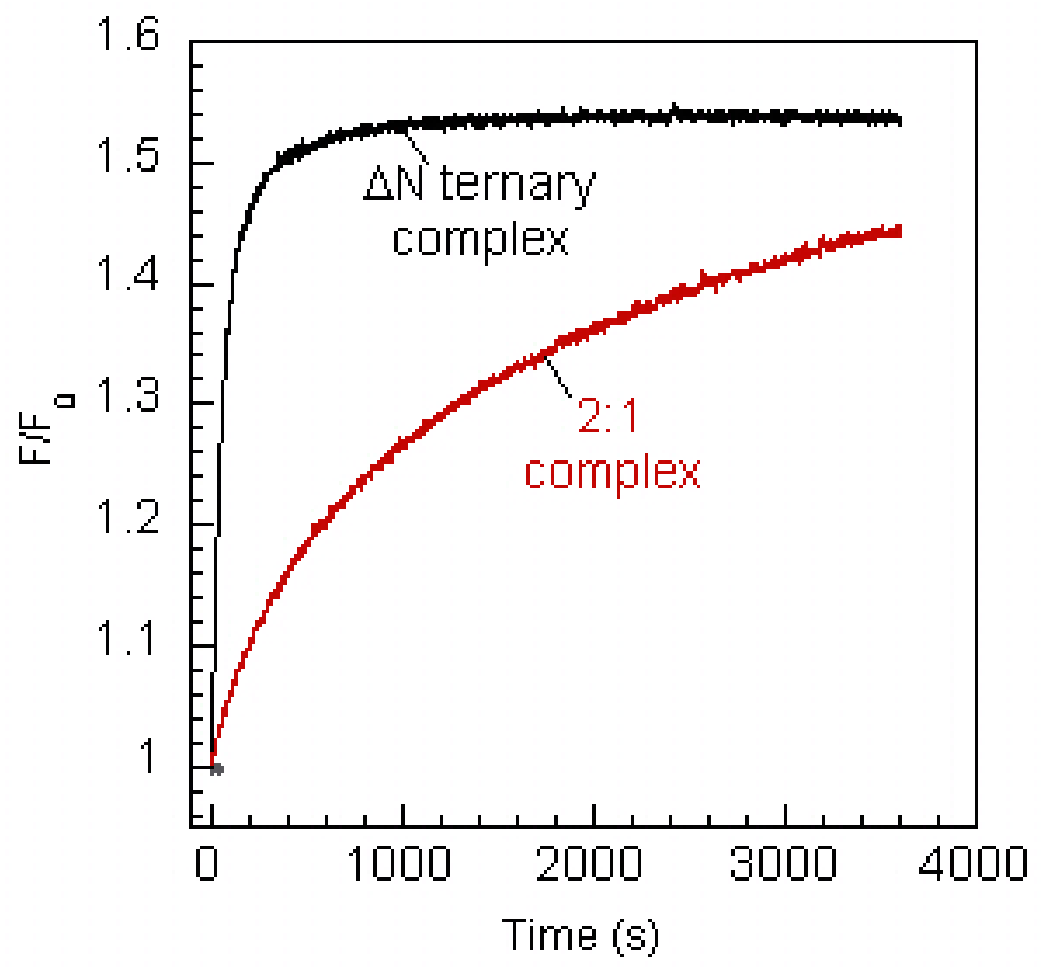

Figure 16 The $\Delta \mathbf{N}$ complex accelerates liposome fusion

The $\Delta \mathrm{N}$ ternary complex containing SNAP-25, the SNARE motif of syntaxin with the transmembrane region (Syx183-288), and Syb49-96 and a binary complex containing Syx183288 and SNAP-25 were purified and inserted into liposome membranes. Fusion was monitored at $30{ }^{\circ} \mathrm{C}$ by lipid dequenching after mixing with NBD/Rhodamine labeled liposomes containing synaptobrevin (Syb1-116). The protein concentration of both liposome populations was about $200 \mathrm{nM}$. 


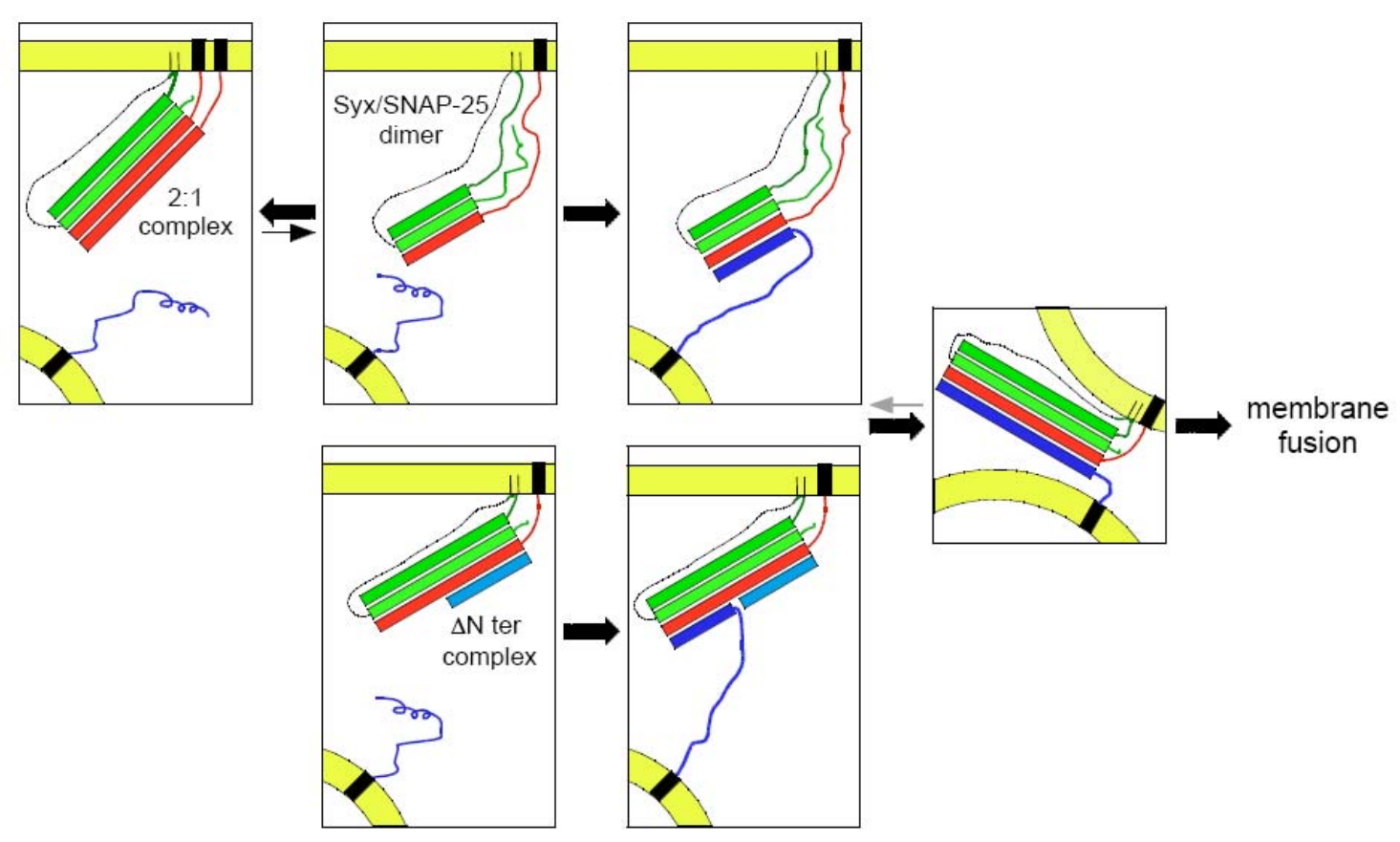

Figure 17 A model of the faster fusion pathway offered by the $\Delta \mathrm{N}$ ternary complex

In solution, the Q-SNAREs syntaxin and SNAP-25 mostly reside as stable 2:1 complex. In this complex the binding site of synaptobrevin is obstructed by a second syntaxin. Binding of synaptobrevin can only occur to the transient syntaxin/SNAP-25 1:1 dimer that offers a free binding site. The competition between syntaxin and synaptobrevin for the same binding site in the syntaxin/SNAP-25 dimer slows ternary SNARE complex assembly. Note that during liposome fusion a second syntaxin can probably even more efficiently compete with synaptobrevin, since the second syntaxin resides in the same membrane. In contrast, the $\Delta \mathrm{N}$ ternary complex prevents the binding of a second syntaxin and at the same time offers a free $\mathrm{N}$-terminal binding site for synaptobrevin. Therefore, synaptobrevin can rapidly bind to the $\Delta \mathrm{N}$ ternary complex. It is possible that the final SNARE zippering process is reversible. To allow for membrane fusion, the $\Delta \mathrm{N}$ synaptobrevin fragment probably needs to be displaced. 
Results

\subsection{Structural basis for the inhibitory role of tomosyn in exocytosis}

Tomosyn is very likely a soluble protein of $130 \mathrm{kDa}$ with a large $\mathrm{N}$-terminal domain containing WD40 repeats and a C-terminal R-SNARE motif. I tried expressing full-length tomosyn as a GST-fusion protein in E.coli. The expressed protein was mainly found in inclusion bodies and it was impracticable to solubilize. However the R-SNARE motif of tomosyn was abundantly expressed and soluble. Therefore all the experiments in this chapter were done only with the R-SNARE motif of tomosyn.

\subsubsection{Structure of the Tomosyn SNARE complex}

The SNARE motif of tomosyn forms a complex after interacting with syntaxin1 and SNAP-25. The ternary complex consisting of the SNARE motif of syntaxin 1A (H3, residues 188-259), the first (Sn1) and second (Sn2) SNARE motif of SNAP-25 (Sn1, residue 7-83 and Sn2, residues 141-204), and the synaptobrevin-like (R) SNARE-motif of tomosyn (residues 1050-1108, numbering based on m-splice variant of tomosyn) was purified.

I used various screens to check for the appropriate condition that would favor crystallization of the tomosyn core complex. Eventually, the crystals of the tomosyn SNARE complex grew under conditions similar to those reported for the core synaptic SNARE complex [25] and a truncated version of this complex [103]. The reservoir solution contained 30\% MPD, 50 $\mathrm{mM} \mathrm{CaCl}_{2}$ and 50mM MES buffer pH-6.0. The crystals diffracted to 2.0- $\AA$ resolution and belong to space group C2. The structure of the tomosyn SNARE complex was solved by molecular replacement using the synaptobrevin SNARE complex (Sb SNARE complex) [103] as a search model and refined to an $R$-value of 16.65\% (see appendix). The final model contained residues 1051-1108 of tomosyn, residues 196-259 of syntaxin, residues 16-83 of Sn1, and residues 142-200 of Sn2. In total, the model contained 252 amino acids and 278 water molecules. (Statistics on data collection and refinement are reported in appendix).

The overall structure of the tomosyn SNARE complex shows an elongated, parallel four-helix bundle similar to the Synaptobrevin SNARE complex (Fig. 18B). A superposition of $\mathrm{C} \alpha$ atoms from the Sb SNARE complex and the tomosyn SNARE 
complex is shown in Fig. 18C. The r.m.s.d. obtained by superposition of the identical chains, i.e. syntaxin residues 200-256, Sn1 residues 27-80, and Sn2 residues 147-199 (in total $164 \mathrm{C} \alpha$ atoms), was only $0.993 \AA$. No significant change was observed when the tomosyn chain was included (r.m.s.d. of $1.059 \AA$; $222 \mathrm{C} \alpha$ atoms). Thus, the two complexes are structurally very similar despite the fact that one of the subunits had been exchanged. Therefore the tomosyn chain occupies the same position as synaptobrevin without causing major conformational rearrangement in the other three subunits.

In the complex, tomosyn, like synaptobrevin, formed comparable number of hydrogen bonds with syntaxin and two SNAP-25 helices. However, the intersubunit salt bridges were differently distributed. Synaptobrevin formed four salt bridges with syntaxin and three salt bridges with Sn2, while tomosyn formed only one salt bridge to syntaxin, and had four salt bridges to Sn2. The difference was even stronger for the intramolecular salt bridges. Six salt bridges stabilized the synaptobrevin helix, and there were 17 salt bridges along the tomosyn helix.

A

$$
\begin{array}{llllllllllllllll}
-7 & -6 & -5 & -4 & -3 & -2 & -1 & 0 & 1 & 2 & 3 & 4 & 5 & 6 & 7 & 8
\end{array}
$$

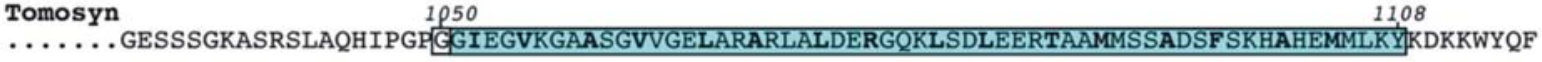

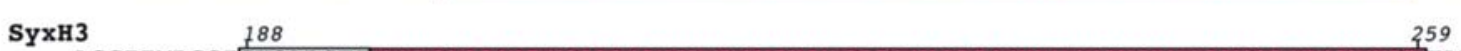

....ASGI IMDSSISKQALSEIETRHSEI IKLENSIRELHDMFMDMAMLVESQGEMIDRIEYNVEHAVDYVERAVSDTKKAVKYQSKRARRKK TMR

$\operatorname{Sn} 1783$

MAEDAD MRIELEEMQRRADQLADESLESTRRMLQLVEESKDAGIRTLVMLDEQGEQLDRVEEGMINHINQDMKEAEKNLKDLGKCCGLF ICPC 、

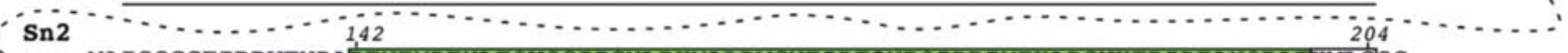

$\because \ldots$ MAISSGGF IRRVTNDARENEMDENLEQVSGIIGNLRHMALDMGNEIDTQNRQIDRIMEKADSNKTRIDEANQRATKMLGSG

B

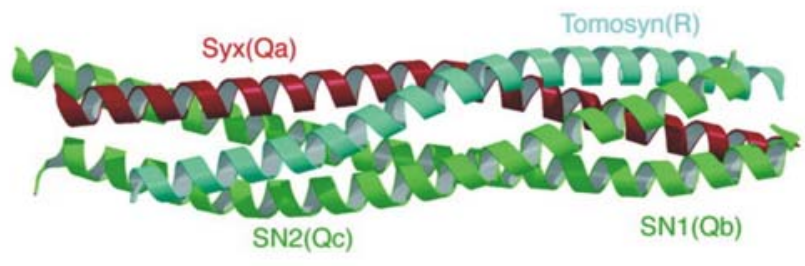

C

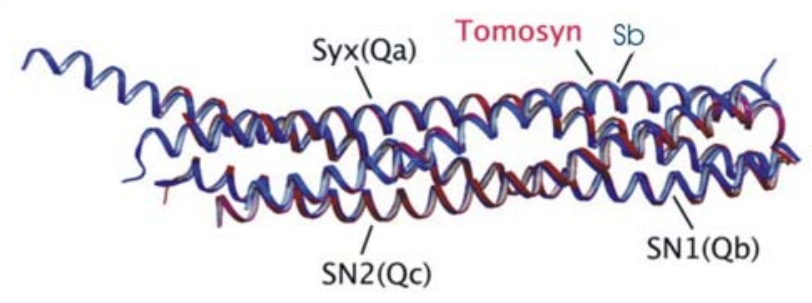


Figure 18 Structure of the Tomosyn SNARE complex

(A), Aligned protein sequences of tomosyn SNARE complex, with tomosyn, first SNARE helix of SNAP-25 (Sn1), second SNARE helix of SNAP-25 (Sn2) and the SNARE motif (H3 domain) of syntaxin (SyxH3). The SNARE layers from -7 to +8 are indicated. The fragments that was used for crystallization is shown inside the boxes. The highlighted residues in the box were the residues that were seen in the electron density map. The dashed line represents the linker region between two SNAP-25 helices. (B) Schematic view of the tomosyn SNARE complex. It was a parallel four helical coiled-coil bundle like the synaptobrevin SNARE complex. (C) C $\alpha$ superposition of tomosyn SNARE complex (red) and neuronal (synaptobrevin containing) SNARE complex (blue). (The crystal structure was solved by Dr.Adelia Razeto).

\subsubsection{The tomosyn SNARE complex is a dead end complex}

Tomosyn does not have a membrane anchor and therefore the tomosyn SNARE complex is probably 'fusion- inactive'. However tomosyn has the same interacting partners as the 'fusion-active' synaptobrevin. A helix exchange between them would mean that tomosyn in the SNARE complex could act as a surrogate for synaptobrevin, and the 'fusion-inactive' tomosyn SNARE complex could be an intermediate for the 'fusion-active' synaptobrevin SNARE complex.

In order to test for helix exchange, I utilized the 'SDS resistance' of the synaptobrevin SNARE complex (Fig. 9A). The complex runs as a single SDS-resistant band. On the other hand, tomosyn SNARE complex was not SDS-resistant and dissociated into monomers under the conditions of SDS-PAGE.

Addition of the SNARE motif of tomosyn to the synaptobrevin SNARE complex did not result in reduction of the SDS-resistant band (Fig 19). Similarly, addition of soluble synaptobrevin (residues 1-96) to the tomosyn SNARE complex did not result in the formation of a SDS-resistant complex (Fig 19). This demonstrates that the synaptobrevin helix in the ternary complex cannot be replaced by an external tomosyn SNARE motif and vice versa. This again confirms that the ternary complex is not in an equilibrium with its monomers [42] and (Section 4.1.1). 


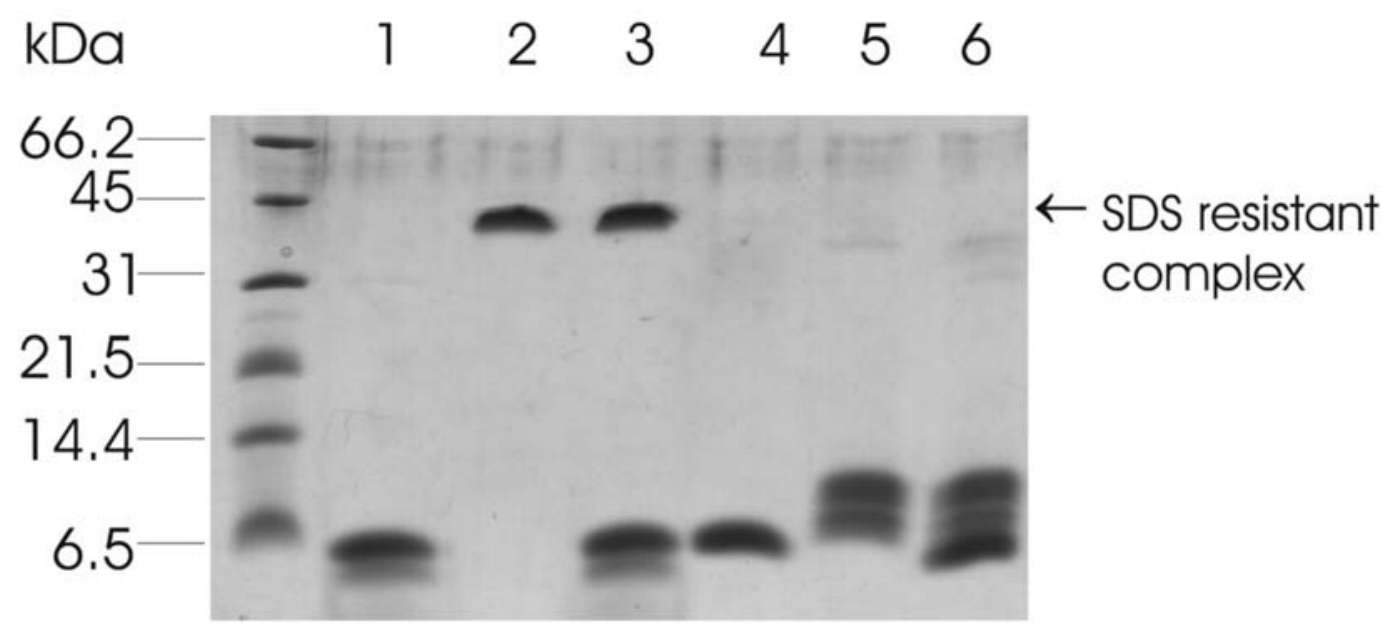

Figure 19 The tomosyn SNARE complex is a dead end complex

The synaptobrevin-containing SNARE complex runs as a single "SDS-resistant" band upon SDSPAGE (lane 2). In contrast, the tomosyn-containing SNARE complex is not SDS-resistant and separates into monomers upon SDS-PAGE (lane 5). Addition of tomosyn to a preformed Synaptobrevin SNARE complex (lane 3) did not, even after over night incubation, result in the disappearance of an SDS-resistant band, indicating that tomosyn did not replace the synaptobrevin helix from the complex. Similarly addition of synaptobrevin to the tomosyn SNARE complex (lane 6) did not result in the appearance of a SDS-resistant band, indicating that synaptobrevin did not replace the tomosyn helix from the complex. Lane 1 contains synaptobrevin and lane 4 contains tomosyn SNARE motif.

As a different approach I used fluorescence anisotropy of Texas Red attached to position 61 on $\mathrm{Sb}$ (1-96) ( $\left.\mathrm{Sb}^{61 \mathrm{TR}}\right)$. To $\mathrm{Sb}^{61 \mathrm{TR}}$, the complementary SNAREs, the H3 domain of syntaxin and SNAP-25, were added. This resulted in an increase in fluorescence anisotropy (Fig. 20), indicating the formation of the ternary complex. After the formation of this complex the tomosyn SNARE motif was added. It did not change the fluorescence anisotropy of $\mathrm{Sb}^{61 \mathrm{TR}}$ (Fig. 20). This indicates that the synaptobrevin helix is not exchanged. Similar results were obtained when unlabeled synaptobrevin (residues 1-96) was added instead of the tomosyn SNARE motif (result similar to Fig. 9B). Similarly, no increase in fluorescence anisotropy was observed when a preformed tomosyn SNARE complex was added to $\mathrm{Sb}^{61 \mathrm{TR}}$ (Fig. 20). Taken together, the synaptobrevin or tomosyn helix in the ternary complex were not replaceable. Therefore 
ternary complexes that contain full- length SNARE motifs are 'dead-end' complexes and NSF activity is probably crucial for the recycling of SNAREs.

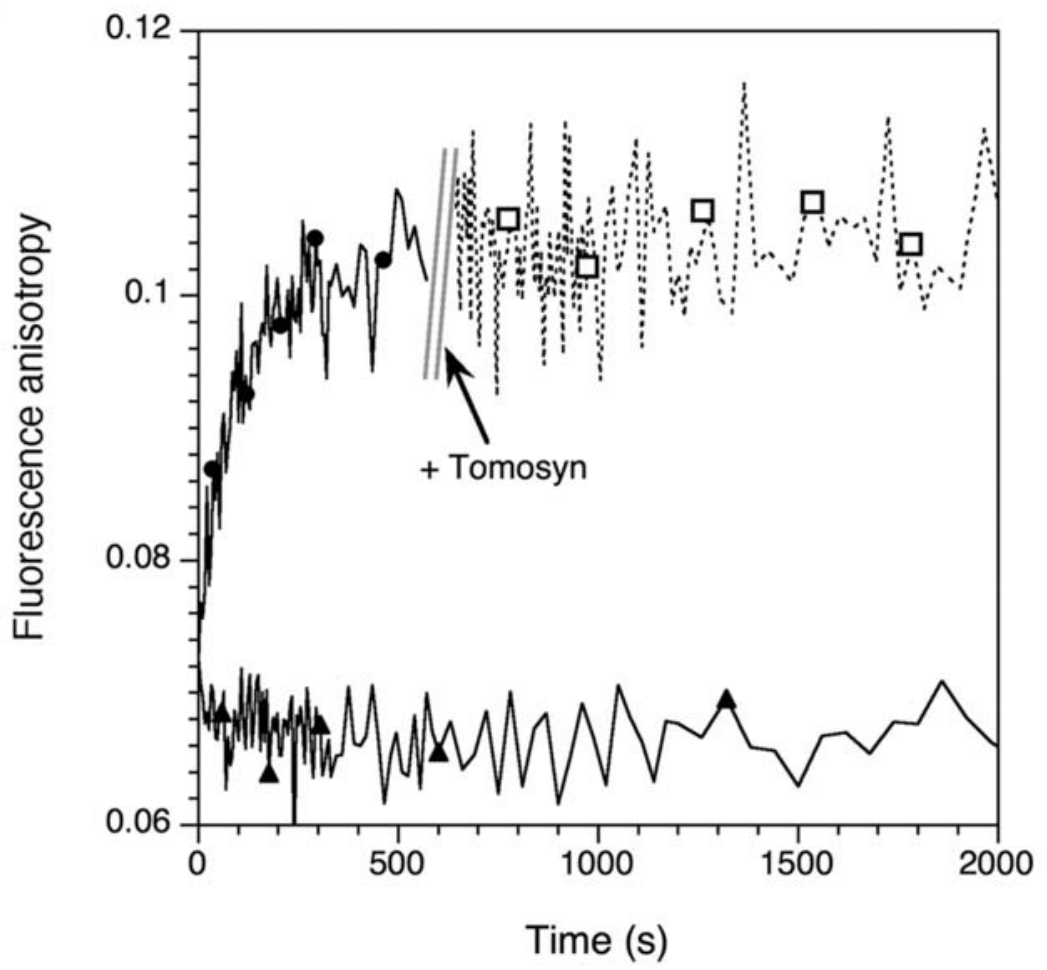

Figure 20 The R-SNARE helix cannot be replaced

The formation of the ternary (synaptobrevin-containing) SNARE complex was followed by fluorescence anisotropy. Upon mixing of SNAP-25 and H3 (2 $\mu \mathrm{M}$, each) with synaptobrevin labeled at position 61 with Texas Red $\left(\mathrm{Syb}^{61 \mathrm{TR}}, \approx 250 \mathrm{nM}\right)$, a clear increase in anisotropy was observed $(\bullet)$, whereas when the preformed tomosyn SNARE complex $(1 \mu \mathrm{M})$ was mixed with Syb $^{61 T R}$ no change occurred $(\boldsymbol{\Delta})$. Likewise, no change in anisotropy was observed when the tomosyn SNARE motif was added, as indicated by an arrow, to the preformed SNARE complex containing $\operatorname{Syb}^{61 \mathrm{TR}}(\square)$.

\subsubsection{Tomosyn and synaptobrevin SNARE complexes assemble at similar rates}

As synaptobrevin and tomosyn SNARE complexes were structurally similar (Fig.18C), it appeared likely that they also use a similar assembly pathway. During the formation of the ternary complex a structural change occurs during which the largely unstructured SNARE monomers transform into a $\alpha$-helical coiled-coil bundle [30]. This structural transformation can be conveniently monitored using a CD spectrophotometer. 
It should be noted that this method does not allow locating the proteins/domains undergoing the structural change.

SNAP-25 was added to a largely non-interacting mix containing syntaxin (residues 180-262) and R-SNARE (either synaptobrevin (residues 30-89) or tomosyn (residues 1051-1116)). Addition of SNAP-25 initiates the formation of ternary complex. The ellipticity decrease reflects the assembly rate of the ternary complex. The rate of increase was similar for synaptobrevin and tomosyn complexes. Therefore both synaptobrevin and tomosyn SNARE complex form at similar rates suggesting that both R-SNARE motifs use the same assembly pathway.

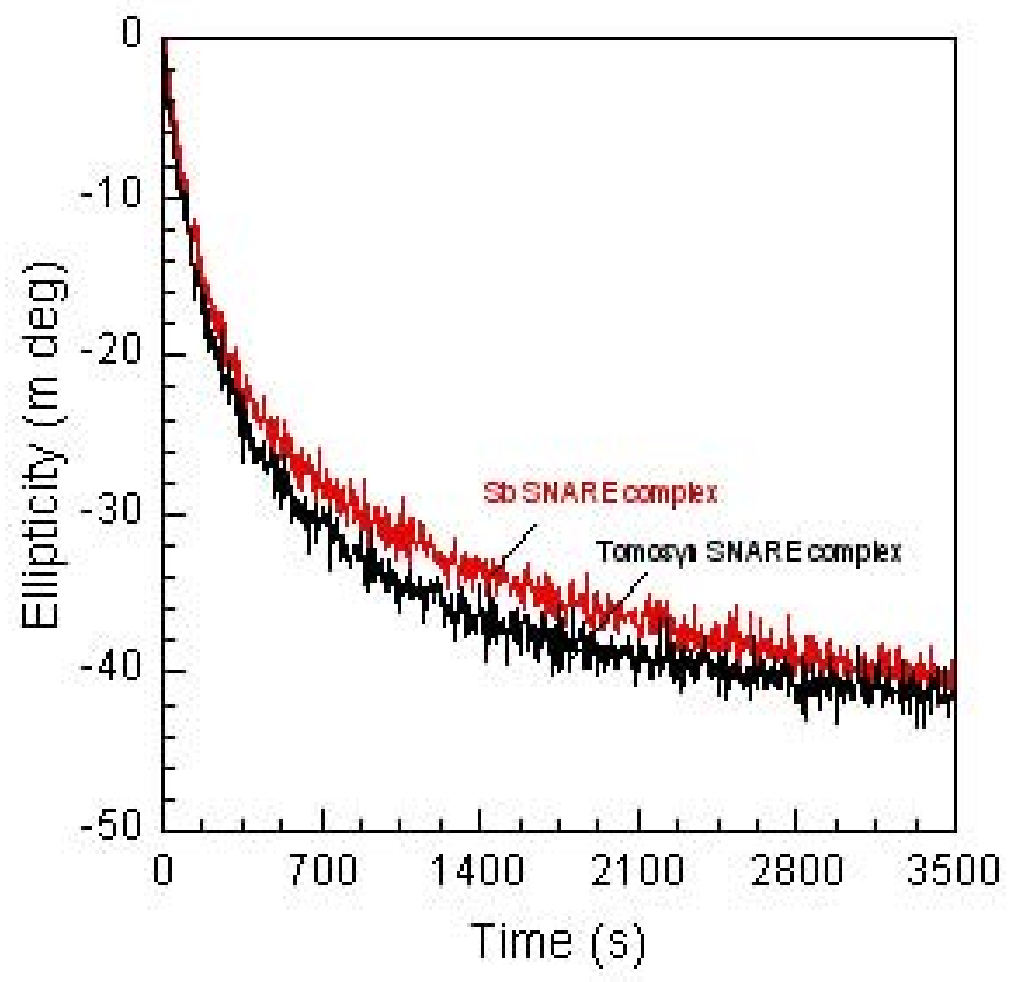

Figure 21 The synaptobrevin and the tomosyn SNARE complex form with similar rates Comparison of the rate of formation of synaptobrevin and tomosyn SNARE complex by observing the change in CD signal at $222 \mathrm{~nm}$, after the addition of SNAP-25 $(1 \mu \mathrm{M})$ to a noninteracting mix of syntaxin $(1 \mu \mathrm{M})$ and synaptobrevin $(1 \mu \mathrm{M})$ (Sb SNARE complex) or to a noninteracting mix of syntaxin and tomosyn $(1 \mu \mathrm{M})$ (Tomosyn SNARE complex). The proteins, upon interaction transform from a largely unstructured form to a highly structured $\alpha$-helical form. The increase in ellipticity over time is the ternary complex formation rate. Both complexes formed at similar rate. 


\subsubsection{Interaction with complexin}

Most differences between synaptobrevin and tomosyn SNARE complex are on the surface of the complex. Therefore it is conceivable that these complexes interact with different partners. Complexin is a soluble protein that interacts with the surface of the synaptobrevin SNARE complex [48, 93, 111, 112]. The central helical region in complexin binds in an anti-parallel orientation to the groove formed by syntaxin and synaptobrevin in the helix bundle. A structural comparison between synaptobrevin and tomosyn SNARE complex suggested that complexin might not be able to strongly bind to the tomosyn SNARE complex (Fig. 22). The interaction between complexin and the synaptobrevin in the ternary complex is through a combination of salt bridges, hydrogen bonds and hydrophobic interactions (For details see Fig. 22). Several of these interactions cannot occur if a tomosyn helix gets substituted for synaptobrevin.

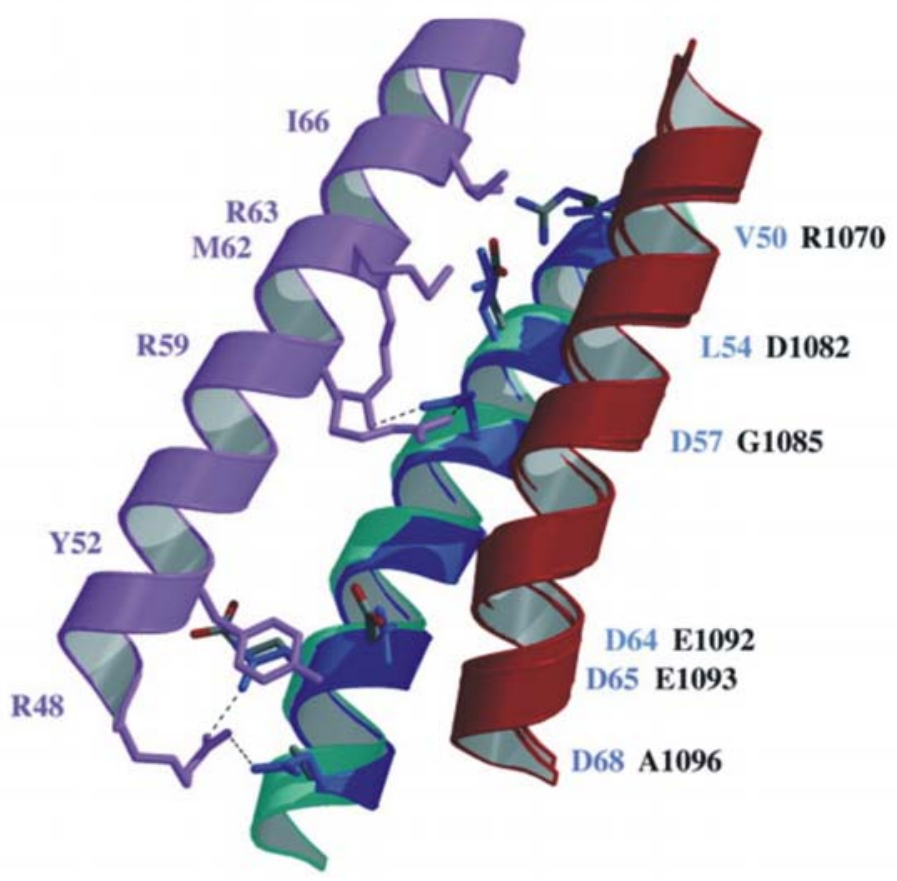

Figure 22 Interaction with complexin

Ribbon diagram of complexin 1 bound to the surface of the neuronal SNARE complex. Complexin (pink) interacts with residues along the groove between the Sb (blue) and Syx (red) helices. For simplification, both SNAP-25 helices are omitted. For structural comparison, Sb was overlaid with tomosyn (sky blue) of the tomosyn SNARE complex. Side chains are shown as sticks in the same color code as the ribbon, except for tomosyn: gray (carbons), red (oxygen), and blue (nitrogen). Salt bridges between Complexin and synaptobrevin side chains are shown as 
dotted lines. There are salt bridges between $\mathrm{Arg}^{48}$ in complexin and residues $\mathrm{Asp}^{68}$ and $\mathrm{Asp}^{65}$ in synaptobrevin. Two complexin residues $\mathrm{Arg}^{59}$ and $\mathrm{Arg}^{63}$ form salt bridges with $\mathrm{Asp}^{57}$ in synaptobrevin. Additionally there is a hydrogen bond between $\mathrm{Tyr}^{52}$ in complexin and $\mathrm{Asp}^{64}$ in synaptobrevin. Furthermore, at the C-terminal end there are hydrophobic interactions between synaptobrevin and complexin which are formed by the complexin residues Met ${ }^{62}, \mathrm{Ile}^{66}$ and the synaptobrevin residues $\mathrm{Leu}^{54}$ and $\mathrm{Val}^{50}$ [48].

Complexin stably interacts with the synaptobrevin SNARE complex and it is shown that the complex runs as a single band in a non-denaturing PAGE [111]. I used the same approach to test whether complexin can interact with the tomosyn SNARE complex. In contrast to synaptobrevin SNARE complex, no complex band was visible, therefore as predicted from the structure analysis complexin did not stably interact with the tomosyn SNARE complex (Fig. 23).

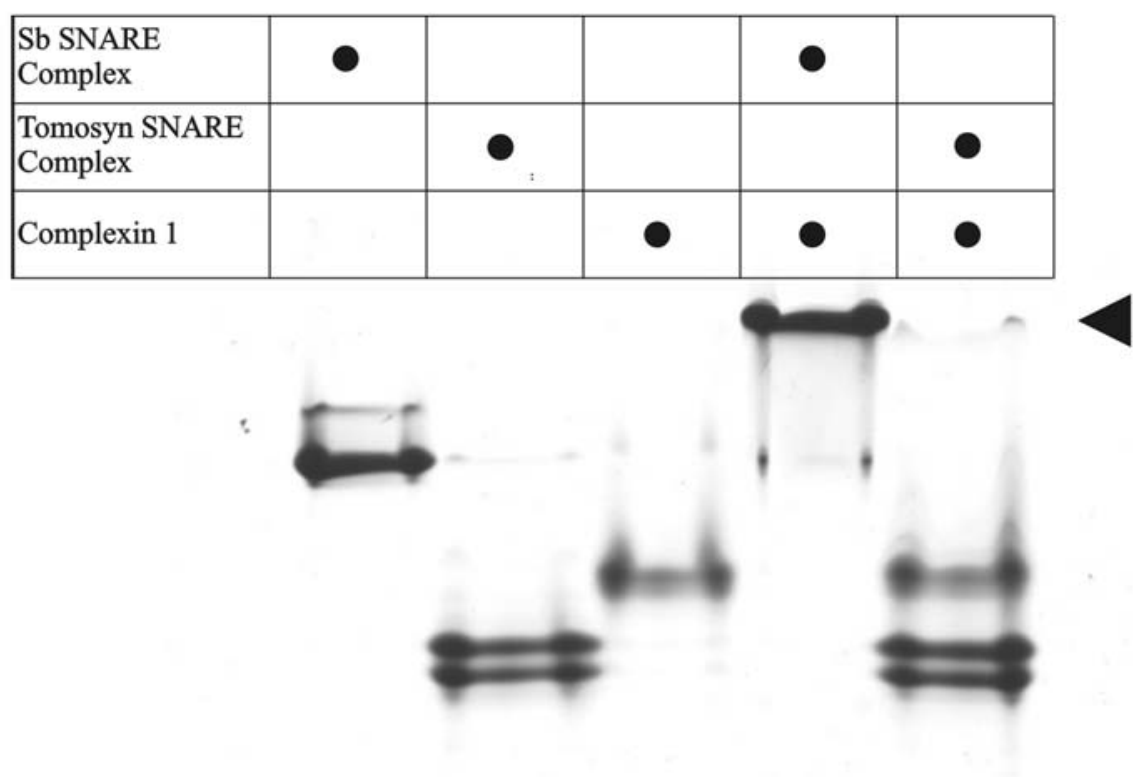

Figure 23 Complexin does not bind to the tomosyn SNARE complex

Complexin has a high affinity interaction with the synaptobrevin (Sb) SNARE complex and the complex is stable after a non denaturing gel electrophoresis (arrowhead). On the contrary complexin did not stably interact with tomosyn SNARE complex. (The tomosyn SNARE complex ran as a double band in the non denaturing gel).

In another approach, a ternary complex, containing either synaptobrevin or tomosyn, was formed using SNAP-25 labeled at position 200 with Texas Red 
(SN25 ${ }^{200 T R}$ ) and the H3 domain of syntaxin. Complexin was labeled at position 39 with Oregon Green $\left(\mathrm{Cpx}^{390 \mathrm{G}}\right)$. In this experiment FRET between $\mathrm{Cpx}^{390 \mathrm{G}}$ and SN25 ${ }^{200 \mathrm{TR}}$ was monitored. A significant quenching of donor fluorescence was observed when $\mathrm{Cpx}^{390 \mathrm{G}}$ was added to the labeled ternary complex containing synaptobrevin (Fig. 24). No significant change was observed when $\mathrm{Cpx}^{390 \mathrm{O}}$ was added to the labeled ternary complex containing tomosyn (Fig. 24). Thus, FRET only occurred between $\mathrm{Cpx}^{390 \mathrm{G}}$ and SN2 $5^{200 T R}$ in the synaptobrevin SNARE complex and it indicates that complexin stably interacts with the synaptobrevin SNARE complex. The lack of significant change after the addition of $\mathrm{Cpx}^{390 \mathrm{G}}$ to the labeled tomosyn SNARE complex suggests that there exists no strong interaction between complexin and the tomosyn SNARE complex.

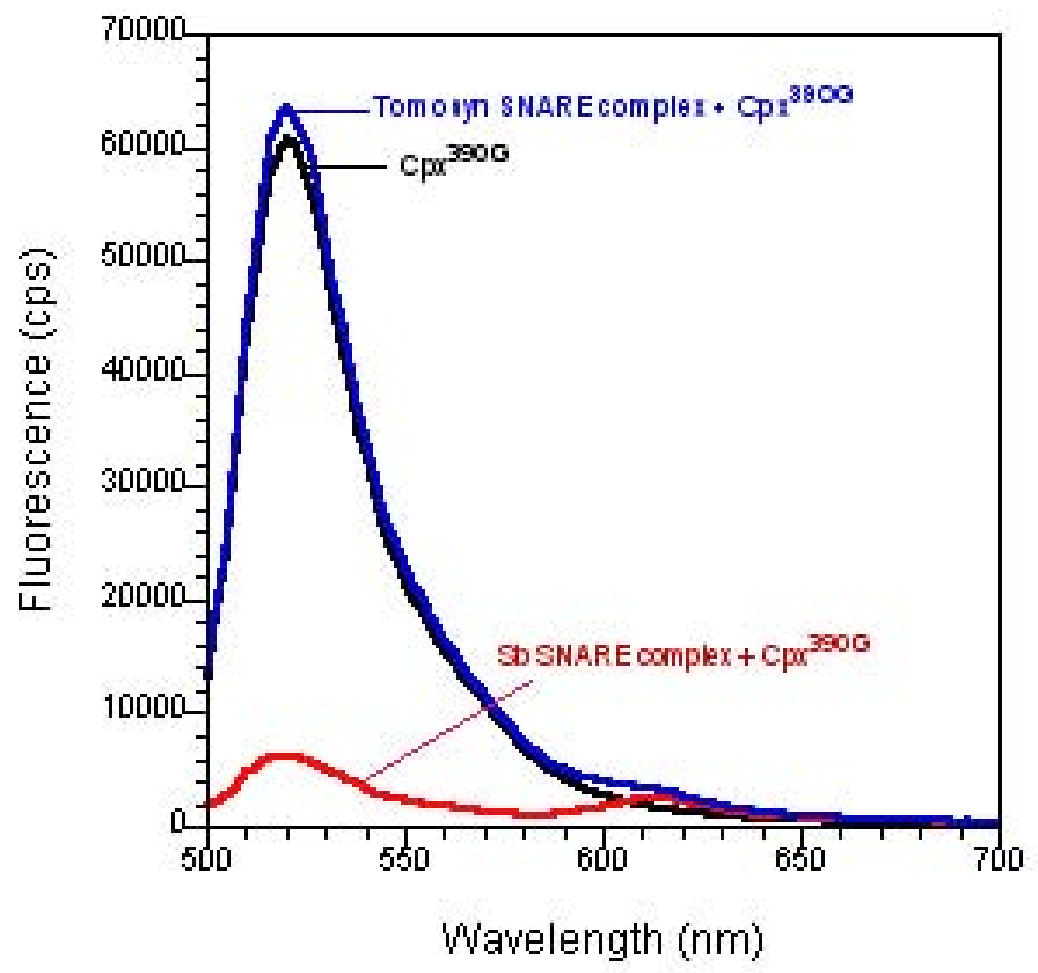

Figure 24 Evaluation of the interaction between complexin and the SNARE complex through FRET

Complexin labeled at position 39 with Oregon green $\left(\mathrm{Cpx}^{390 \mathrm{O}}\right)$ was added either to tomosyn SNARE complex or to synaptobrevin (Sb) SNARE complex. These complexes contained SNAP25 labeled at position 200 with Texas red (SNAP-25 $5^{200 T R}$ ). FRET occurred between $\mathrm{Cpx}^{390 \mathrm{O}}$ and SNAP- $25^{200 T R}$, due to the interaction of complexin with the synaptobrevin SNARE complex and it caused a pronounced decrease in donor fluorescence. However no such change was observed 
when $\mathrm{Cpx}^{390 G}$ was added to tomosyn SNARE complex suggesting that complexin does not interact with tomosyn SNARE complex. The spectrum of complexin alone is shown in black.

\subsubsection{Tomosyn is evolutionarily conserved}

Tomosyn was suggested to function in neuronal exocytosis [39]. However there are splice variants in tomosyn that are expressed outside the nervous system [79, 80]. This hinted that tomosyn homologues are perhaps present in other kingdoms and it could have a broader function in vesicular trafficking. Our data bank searches revealed several new homologues of tomosyn (Fig. 25). Surprisingly, tomosyn was also present in plants and fungi. All the homologues of tomosyn had a similar domain structure; they possess an N-terminal domain with WD 40 repeats and a C-terminal R-SNARE motif.

All the tomosyn homologues that we analyzed, with the exception of A. thaliana (tom2-AT), had an arginine in the zero layer. Tom2-AT has an asparagine in the zero layer and a charged residue, glutamate in the putative -4 layer. The presence of glutamate in a layer is also seen in the endosomal SNARE complex [47], where layer 6 in syntaxin 8 is glutamate. Fungi have tomosyn homologs containing an R-SNARE motif. The yeast proteins, Sro7p and Sro77p [76] has been described as Lgl-homologues without the RSNARE motif. A closer analysis revealed that they contain a C-terminal portion of the SNARE motif and are to be considered as tomosyn homologues. In our analysis, several fungi homologues also contained charged residues in the layer. It is not clear if they can form a stable ternary complex. Tomosyn is conserved through eukaryotes and it probably has a broader function in vesicular trafficking. 


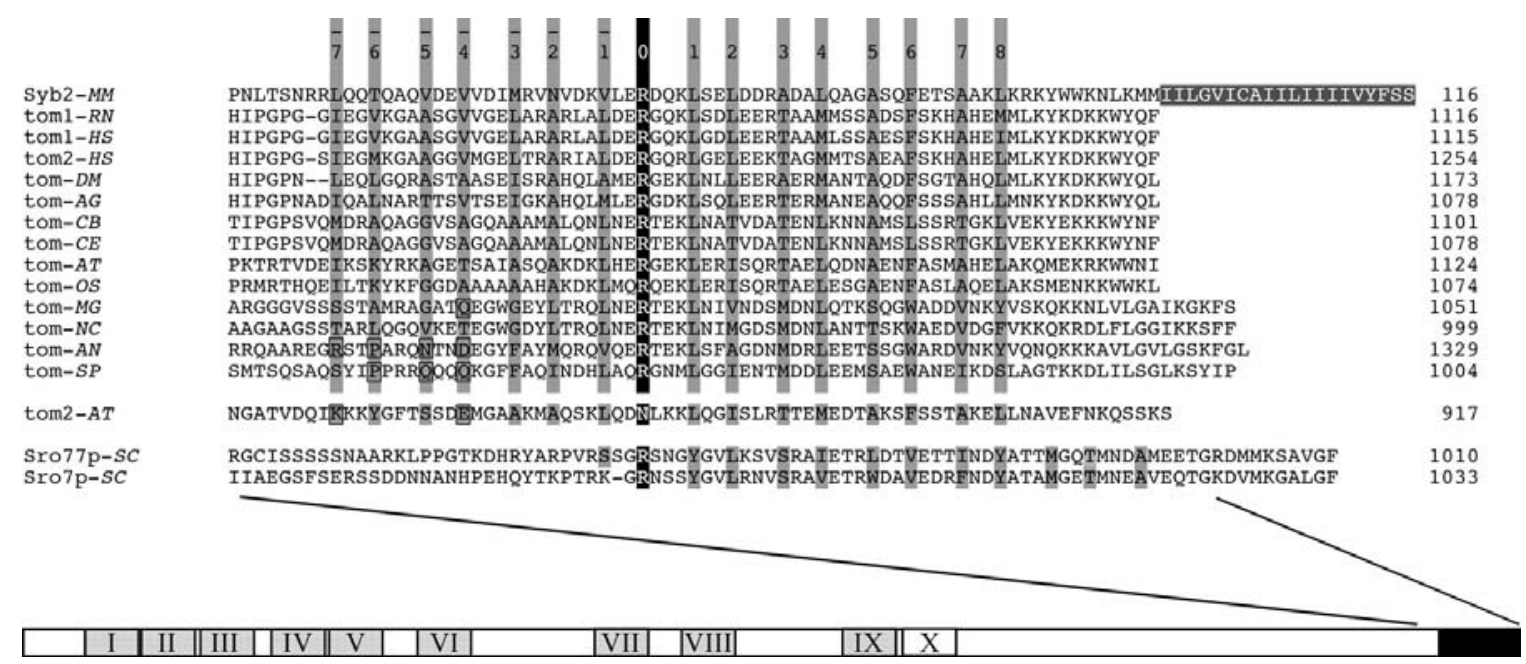

Figure 25 Tomosyn is evolutionarily conserved

The R-SNARE motif of synaptobrevin 2 (Mus musculus: gi 207626, Syb2-MM) and several tomosyn homologues from the following species were aligned: Rattus norvegicus (gi 13540648, tom1-RN); Homo sapiens (gi 31652247, tom1-HS, and gi 41146546, tom2-HS); Caenorhabditis elegans (gi 17508275, tom-CE); Caenorhabditis briggsae (gi 39598218, tom-CB); Drosophila melanogaster (gi 28571171, tom-DM); Anopheles gambiae (gi 31204405, tom-AG); Magnaporthe grisea (gi 38102429, tom-MG); Neurospora crassa (gi 32423373, tom-NC); Aspergillus nidulans (gi 40741778, tom-AN); Schizosaccharomyces pombe (gi 19113918, tomSP); Oryza sativa (gi 34898238, tom-OS). A. thalania (gi 15239173, tom1-AT, and gi 15237037, tom2-AT). The transmembrane region at the $\mathrm{COOH}$ terminus of synaptobrevin 2 is indicated white on gray background. The number of the layers is indicated on the top. The central 0-layer containing the highly conserved arginine is indicated white on a black background. Other layer residues are shown in black on a gray background. Note that one Arabidopsis tomosyn (tom2$A T$ ) and several Fungi tomosyn homologs have hydrophilic residues (boxed) in putative heptad repeat layers upstream of the 0-layer. It is unclear whether these residues can form a hydrophobic interior of a SNARE helix bundle. It is possible that a stutter or a skip can explain the apparent break in the heptad repeat periodicity of these homologs. We also aligned the COOH-terminal end of the two tomosyn-like proteins Sro7p (gi 6325289, Sro7p-SC) and Sro77p (gi 6319362, Sro77p-SC) from Saccharomyces cerevisiae to indicate that these proteins might still possess a partial R-SNARE motif. A schematic depiction of the domain structure of tomosyn is given underneath. The $\mathrm{COOH}$-terminal R-SNARE motif is shown in black. Putative WD repeats in the $\mathrm{NH}_{2}$-terminal region are boxed. (The alignment was done by Dr.Dirk Fasshauer). 


\subsection{Characterization of Amisyn}

\subsubsection{Protein purifications}

Amisyn is a soluble protein of $25 \mathrm{kDa}$, like tomosyn it contains an $\mathrm{N}$-terminal domain and a C-terminal R-SNARE motif. The N-terminal domain is similar to the C.elegans homologue of the tethering protein Uso1p [87] and also has limited homology $\sim 32 \%$, with a Sec3-like protein (Sec3 is also implicated in tethering). At the C-terminus, amisyn contains an R-SNARE motif. The full-length amisyn, Ami-fl (residues 1-210) and the version that contains only the SNARE motif of amisyn, Ami-SN (residues 162-210) was cloned as described in the Materials and Methods section. The full-length amisyn is reported to be poorly expressed and insoluble [88]. Ami-fl was also poorly expressed under our expression conditions but it was nevertheless soluble. Both Ami-fl and AmiSN were initially purified using $\mathrm{Ni}^{2+}$-NTA affinity chromatography, further purification was done on a Mono-S column. The proteins Ami-fl and Ami-SN, as assessed by SDSPAGE, were more than $95 \%$ pure. Furthermore, by mass spectrometry analysis I confirmed the 25kDa band as Ami-fl (data not shown).

A

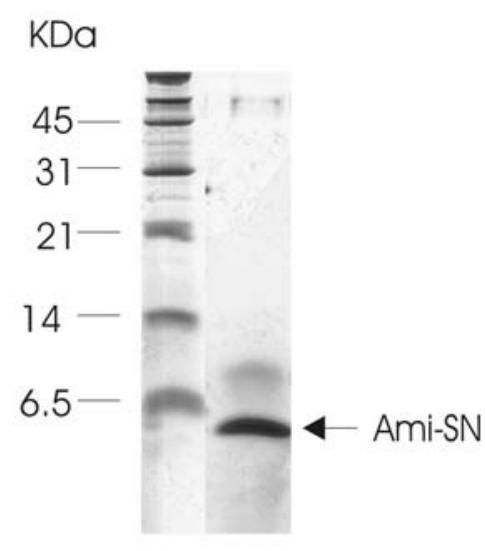

B

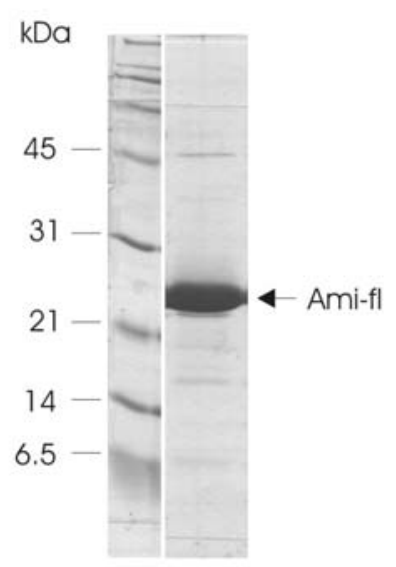

Figure 26 Purification of full-length and SNARE motif of amisyn SDS gel to evaluate the purity of the SNARE motif of Amisyn (Ami-SN) and the full-length amisyn (Ami-fl). 


\subsubsection{Formation of the ternary complex}

The SNARE motif of amisyn interacts with the Q-SNAREs syntaxin1 and SNAP25 and forms a 'SDS-resistant' ternary complex [88]. Here I show that both, the SNARE motif (Ami-SN) and the full-length amisyn (Ami-fl), formed a 'SDS-resistant' ternary complex with the neuronal Q-SNAREs.

I attempted to crystallize these complexes in order to determine their structure by X-ray crystallography. Crystal screen1 and 2, PEG/Ion screen and Grid Screen MPD (Hampton research) were used to test for appropriate crystallizing condition. Unfortunately none of these conditions yielded crystals.

A
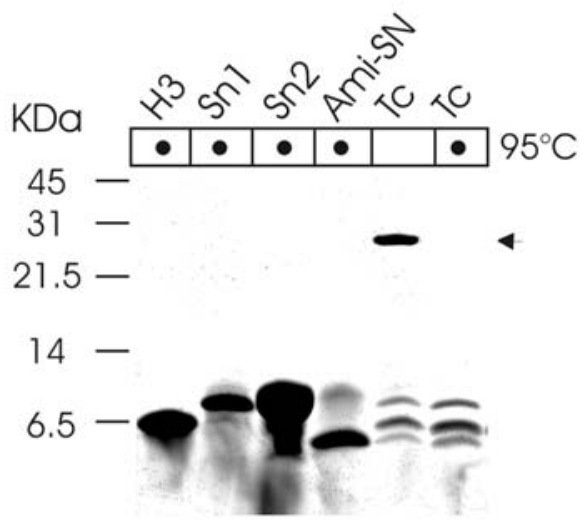

B

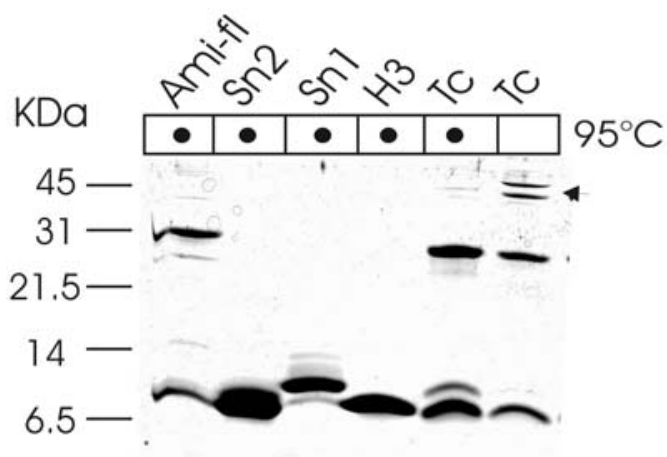

Figure 27 Formation of the ternary complex

(A) The SNARE motif of amisyn (Ami-SN), after interacting with the Q-SNARE motifs syntaxin-H3 (H3), the first helix of SNAP-25 (Sn1) and the second helix of SNAP-25 (Sn2) formed a 'SDS-resistant' ternary complex (Tc) (shown by arrowhead). (B) As before, the fulllength amisyn (Ami-fl) also formed a ternary complex after interacting with the Q-SNAREs. In both the cases the complex dissociated after heating.

\subsubsection{Q-SNARE interaction}

A ternary complex was formed after the interaction of the SNARE motif of amisyn with the neuronal Q-SNAREs (Fig. 27). To obtain a read-out for amisyn complex formation I labeled the single cysteine at the end of amisyn with Oregon Green. The fluorescence anisotropy of labeled Ami-SN (100nM) was measured. The fluorescence anisotropy was unaltered after the addition of H3 domain of syntaxin or SNAP-25. However an increase in anisotropy was observed when both H3 and SNAP-25 were 
added to the labeled Ami-SN. It suggests that in the presence of both neuronal QSNAREs, Amisyn- Q-SNARE interaction is potentiated.

In another approach, the $\mathrm{H} 3$ domain of syntaxin was labeled at position 197 with Oregon Green $\left(\mathrm{H}^{1970 G}\right)$. The fluorescence anisotropy of $\mathrm{H}^{1970 \mathrm{G}}$ was measured; it did not change after the addition of Ami-SN. However addition of both Ami-SN and SNAP25 caused an increase in the fluorescence anisotropy. It appears that the SNARE motif of amisyn interacts with the Q-SNAREs as do synaptobrevin or the SNARE motif of tomosyn.

A

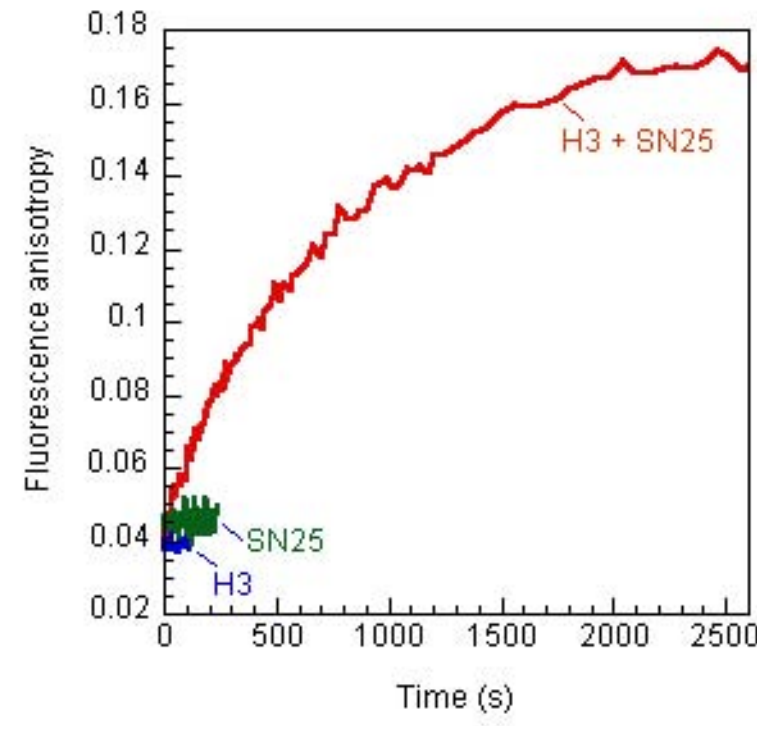

B

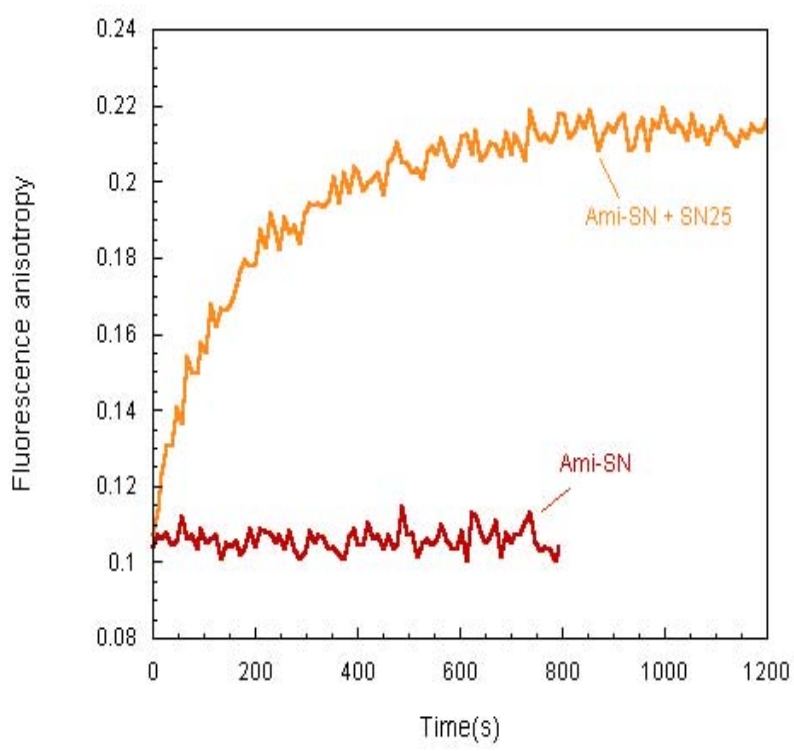

Figure 28 Q-SNARE interaction

(A) The fluorescence anisotropy of the SNARE motif of amisyn (100nM), labeled at position 210 with Oregon Green, did not change after the addition of $\mathrm{H3}$ domain of syntaxin $(\mathrm{H} 3,1 \mu \mathrm{M})$ or SNAP-25 (SN25) $(1.5 \mu \mathrm{M})$. However an increase in fluorescence anisotropy was observed when both H3 and SN25 were added. (B) Similarly, the fluorescence anisotropy of H3 domain of syntaxin, labeled at position 197 with Oregon Green $\left(\mathrm{H} 3^{1970 \mathrm{G}}, \sim 250 \mathrm{nM}\right)$, did not change after the addition of the SNARE motif of amisyn (Ami-SN, $2 \mu \mathrm{M})$. An increase in anisotropy was observed when both Ami-SN $(2 \mu \mathrm{M})$ and SNAP-25 (SN25) $(2 \mu \mathrm{M})$ were added to H3 ${ }^{1970 G}$. In both the experiments, the increase in fluorescence anisotropy is indicative of ternary complex formation. 
In another approach I used CD spectroscopy to monitor complex formation. I found a characteristic $\alpha$-helical spectrum, with minima at 208 and 222nm, after the addition of the SNARE motif of amisyn to SNAP-25 and the H3 domain of syntaxin (Fig. 29). The monomers on the other hand were largely unstructured (Fig. 29). Therefore similar to what is seen in synaptobrevin [30] and tomosyn SNARE motif [81], structural change also occurs after the formation of amisyn SNARE complex.

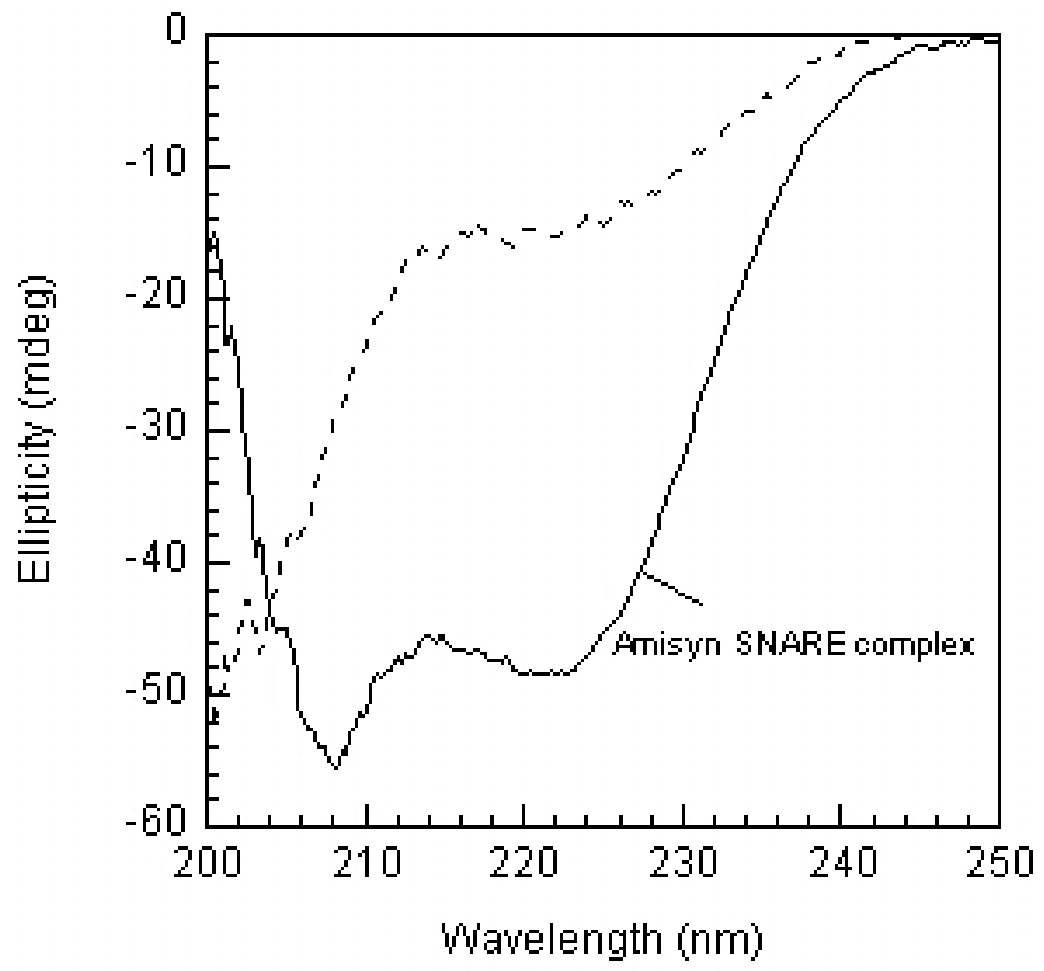

Figure 29 Structural changes after the formation of amisyn SNARE complex

The SNARE motif of amisyn interacted with SNAP-25 and H3 domain of syntaxin and formed a $\alpha$-helical SNARE complex. The dotted line represents theoretical non-interacting spectrum of the monomers.

\subsubsection{Sub-cellular distribution of Amisyn}

Polyclonal antibody was raised against full-length amisyn. It was affinity purified and the antibodies recognized amisyn in the native and recombinant form. The affinity purified antibody was used to investigate the distribution of amisyn in sub-cellular fractions of the neuronal tissue. Amisyn was largely absent in the supernatant fractions $\mathrm{S}_{3}$ and $\mathrm{LS}_{2}$ and was enriched in synaptosomal membranes $\left(\mathrm{LP}_{1}\right)$ and in the pellet containing 
crude synaptic vesicles $\left(\mathrm{LP}_{2}\right)$ (Fig. 30). However amisyn was not found on the synaptic vesicles that were further purified by sucrose density gradient centrifugation (SGV) (Fig. 30). Therefore the data suggests that amisyn is enriched on the membranes in the nerve terminals but not present on the synaptic vesicles. It should be noted that amisyn neither contain membrane anchor nor lipidation recognition motif. Therefore amisyn might be indirectly attached to the membrane due to its interaction with the membrane proteins, syntaxin and SNAP-25 or is perhaps attached to the cytoskeleton.

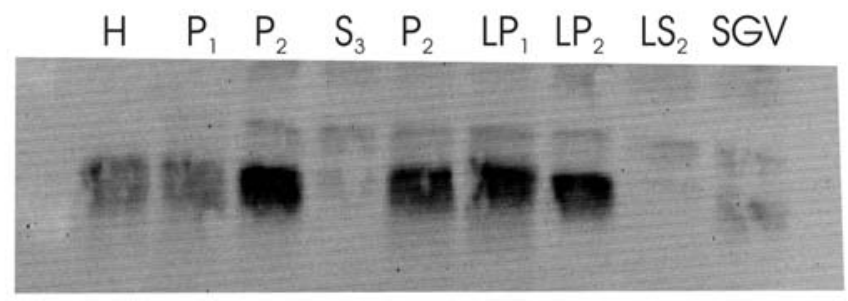

Figure 30 Sub-cellular distribution of amisyn

$\mathrm{H}$ - rat brain homogenate; $\mathrm{P}_{1}$ - Pellet after $800 \mathrm{~g}$ centrifugation, the supernatant was centrifuged at $9200 \mathrm{~g}$ for $15 \mathrm{~min}$ yielding pellet $\mathrm{P}_{2}$ (crude synaptosomal pellet) and supernatant $\mathrm{S}_{2}$. $\mathrm{S}_{2}$ was centrifuged at 160,000 g to get supernatant $S_{3}$ and pellet $P_{3}$. The synaptosomes $\left(\mathrm{P}_{2}\right.$ pellet) were osmotic ally lysed and homogenized. They were centrifuged at 33,000 g for $20 \mathrm{~min}$ to yield pellet $\mathrm{LP}_{1}$ (which contains synaptosomal membranes) and supernatant $\mathrm{LS}_{1}$. LS $\mathrm{S}_{1}$ was centrifuged at 260,000 g for 2 hours to get pellet LP2 (which contains crude synaptic vesicles) and supernatant $\mathrm{LS}_{2}$. The $\mathrm{LP}_{2}$ was run on a sucrose gradient to yield SGV, which contains pure synaptic vesicles. Amisyn was largely present in membrane fractions and was enriched in synaptosomal membranes (LP1). It was however absent on pure synaptic vesicles (SGV). 


\section{Discussion}

\subsection{Helix replacement mechanism sheds new light on SNARE mediated fusion}

High speed and intricate regulation is the hallmark of neuronal exocytosis. Immense contributions from the fields of cell biology, genetics, physiology and biochemistry are leading to a better understanding of the molecular basis for neuronal exocytosis. Several studies demonstrate that SNAREs play an important role in this process. For instance, genetic ablation of SNAREs [50-52], cleavage of SNAREs using neurotoxins [56-60, 66, 113], interfering with the assembly of SNAREs using antibodies [49] or mutations in SNAREs [53-55] alter the exocytotic behaviour of the cells. Syntaxin1, SNAP-25 and synaptobrevin are the SNAREs dedicated to neuronal exocytosis.

A relatively simple scenario for the role of SNAREs in neuronal exocytosis is given by the zipper model, which proposes that assembly occurs between synaptobrevin on the vesicle membrane and the syntaxin and SNAP-25 on the pre-synaptic plasma membrane. The assembly is assumed to proceed in a zipper-like fashion from $\mathrm{N}$ to $\mathrm{C}$ terminus; from the distal end of these proteins towards their membrane anchors. The energy released during the assembly could possibly be sufficient to overcome the energy barrier for fusing membranes.

SNAREs act as minimal fusion machines because neuronal SNAREs when reconstituted, are able to fuse liposomes [65]. However, SNARE mediated liposome fusion occurs in the order of minutes, whereas neurotransmitter release occurs in a millisecond time-scale. In this study I showed that the sluggishness in the liposome fusion experiments was largely due to an `off-pathway' reaction.

\subsubsection{Helix replacement}

The assembly among the neuronal SNAREs syntaxin, SNAP-25 and synaptobrevin culminates in a highly stable ternary complex. The ternary complex, once formed, does not spontaneously dissociate, there is no equilibrium between the ternary complex and the unassembled monomers. This is apparent from the marked hysteresis in the folding and unfolding transitions of the ternary complex [42], which also suggests 
that the assembly and disassembly employ different routes. Synaptobrevin fragments with $\mathrm{N}$ or C-terminal truncations also formed stable ternary complexes (Fig. 9A) and most of these shortened synaptobrevin complexes also exhibited hysteresis (data not shown) suggesting the absence of spontaneous dissociation in these complexes.

In line with these findings, an externally added synaptobrevin did not replace the full-length soluble synaptobrevin (residues 1-96) in the ternary complex (Fig. 9B). Surprisingly, an externally added full-length soluble synaptobrevin replaced the synaptobrevin fragment in most of the shortened synaptobrevin complexes (Fig. 9A\&B). Therefore replacement, as observed in most of these shortened synaptobrevin complexes was due to an active mechanism.

My experiments revealed that for a replacement to take place the externally added synaptobrevin has to bind to the shortened complex. As shortened synaptobrevin complexes lack certain portions of the synaptobrevin SNARE motif, a region exists in the complexes, which is composed of only syntaxin and SNAP-25. This is the region to which the replacing synaptobrevin bind (Fig. 13). Further analysis showed that in $\Delta \mathrm{N}$ ternary complex this region was structured (Fig. 14A) and offered a 'readily-available ' binding site that enabled rapid binding of the replacing synaptobrevin. On the contrary, in $\Delta \mathrm{C}$ ternary complex this region acquired $\alpha$-helical structure upon interacting with the synaptobrevin (Fig. 14B), indicating that the binding site was not 'readily-available' and therefore binding was slow.

To get more insights into the replacement mechanism, I focussed on two $\Delta \mathrm{N}$ ternary complexes Tc (42-96) and Tc (49-96) (Tc - ternary complex). Externally added Sb (42-96) did not replace the synaptobrevin fragment in Tc (42-96); similarly externally added Sb (49-96) did not replace the synaptobrevin fragment in Tc (49-96) (Fig. 11\&12). This demonstrates that the synaptobrevin fragment in the ternary complex does not spontaneously dissociate. Similarly, Sb (35-96) also was not able to replace the synaptobrevin from either of these shortened synaptobrevin complexes. Replacement, however, occurred actively when the N-terminus of the synaptobrevin SNARE motif was intact (Fig. 11\&12). It is very likely that the Sb (35-96) fragment does not contain enough $\mathrm{N}$-terminal residues to enable efficient binding, and replacement cannot occur without 
efficient binding. The N-terminal residues therefore enable efficient binding and indirectly influence the downstream replacement reaction. An analysis of the replacement kinetics by various external synaptobrevin fragments that contained intact $\mathrm{N}$-terminui but variable lengths in the C-termini showed that replacement kinetics was reduced by decreasing the C-terminal length of the replacing synaptobrevin (Fig. 11 \& 12). Furthermore, shortening the synaptobrevin fragment in the complex enhanced the replacement rate (Fig. 11\&12).

Taken together, my data support that short synaptobrevin fragments in the ternary complex are actively replaced. Replacement appeared to take place in two steps, first the binding of the externally added synaptobrevin, followed by an active displacement of the short synaptobrevin, but the exact displacement mechanism remains to be clarified. To my knowledge this is the first report about an active helix exchange mechanism in a stable coiled-coil bundle.

\subsubsection{Synaptobrevin binding}

Soluble synaptobrevin (residues 1-96) rapidly occupied the 'readily-available' binding site at the $\mathrm{N}$-terminus of the $\Delta \mathrm{N}$ ternary complex (Fig. 10). Synaptobrevin did not bind to Tc (35-96), suggesting that this complex lacked a 'readily-available' binding site. However, remarkably, shortening the synaptobrevin in the complex by only seven more amino acid residues, i.e. two coiled-coil turns, as in Tc (42-96), enabled rapid binding of synaptobrevin. It is reasonable to assume that the interactions between the neuronal SNAREs are confined to the SNARE motifs. The SNARE motif of synaptobrevin starts from residue 28. Rapid binding was already seen in Tc (42-96), therefore the 'readily-available' binding site appears to be composed only of a short stretch of amino acids. The binding site is probably not specific for R-SNARE motifs, as endobrevin, a homologue of synaptobrevin involved in endosomal trafficking, is able to replace Sb (42-96) from the ternary complex with similar efficiency as Sb (1-96) (Fig. $11)$. 
However, it is likely that the residues upstream to the SNARE motif are also involved during rapid synaptobrevin binding. The Sb (1-59) fragment blocks the binding of soluble synaptobrevin (residues 1-96) to the $\Delta \mathrm{N}$ ternary complex (Fig. 13), whereas a peptide (residues 28-41) did not prevent soluble synaptobrevin binding to Tc (49-96). There could be two possible reasons for the inability of the peptide to block synaptobrevin binding; first, the peptide may not effectively bind to the 'readilyavailable' binding site. Second, after binding of the peptide synaptobrevin could bind to the region upstream of the SNARE motif and rapidly replace the peptide. Both possibilities suggest that residues upstream to the SNARE motif might be involved during synaptobrevin binding to the $\Delta \mathrm{N}$ ternary complex.

Biochemical studies have shown that the SNARE assembly initiates with a transient 1:1 interaction between syntaxin and SNAP-25 [45]. The N-terminal region in the SNARE motifs of syntaxin and SNAP-25 is absolutely essential for the formation of such a transient dimer. The formation of this dimer is the rate-limiting step in SNARE assembly. This dimer is probably the true acceptor site for the vesicular synaptobrevin, as synaptobrevin can bind rapidly to this dimer to form the ternary complex (Dr.Dirk Fasshauer, unpublished observations).

Shortened synaptobrevin complexes could be used to evaluate the binding mode of synaptobrevin to the 1:1 dimer. As discussed above, these complexes lack part of the synaptobrevin SNARE motif and these regions contain syntaxin and SNAP-25 in 1:1 stoichiometry. The rapid binding of synaptobrevin to the 1:1 dimer is similar to the rapid binding of synaptobrevin to the $\Delta \mathrm{N}$ ternary complex. Therefore it is reasonable to assume that the 1:1 dimer offers a similar 'readily-available' site as the N-terminal region of the $\Delta \mathrm{N}$ ternary complex. The $\Delta \mathrm{N}$ ternary complex could be considered as a substitute for the 1:1 dimer.

Few studies suggest that in synaptobrevin the residues proximal to the transmembrane anchor are inserted into the membrane [114-116]. EPR accessibility measurements suggest that the residues 85-92 are inserted in the membrane and form two $\alpha$-helical turns [115]. It was proposed that this helical segment might serve as a seed for coiled-coil formation and therefore assembly could initiate from the C-terminus of 
synaptobrevin. However, in my study, assembly is rapid only when initiated from the Nterminus (Fig. 10) and a C-terminal assembly is probably inappropriate for a rapid reaction like exocytosis.

As formation of this 1:1 dimer is the rate-limiting step in SNARE assembly it seems likely that the role of so called priming factors is to enhance or to modulate the formation of this acceptor site. Thereby, priming factors by controlling the formation of this acceptor site could modulate exocytosis.

\subsubsection{The release-ready states}

To accomplish rapid exocytosis neurons and neurosecretory cells keep vesicles in a 'release-ready' state. A few studies suggest that before $\mathrm{Ca}^{2+}$ influx the fusion machinery (SNAREs) might reside in a partially zippered state $[49,66,67]$ in which only the Nterminal residues of the SNAREs are assembled. Keeping SNAREs in a partially zippered state is an attractive mechanism by which neurons and neuroendocrine cells can accomplish rapid exocytosis and this could be the molecular nature of the 'release-ready' state. However these studies do not directly monitor SNAREs and it is still ambiguous if SNAREs actually reside in such a state. In this study I directly analysed SNARE assembly using spectroscopical methods. My data suggests that two configurations of 'release-ready' states are possible.

Configuration 1:

In this model, the SNARE proteins between opposing membranes are still without contact. The Q-SNAREs have already formed a binding site for synaptobrevin and are kept in a 1:1 configuration (Fig. 31). My results show that synaptobrevin is able to quickly engage with this Q-SNARE acceptor site (Fig. 10). Calcium regulation could also be envisioned in this pathway. A calcium binding protein could bind to the $\mathrm{N}$ terminus of synaptobrevin or 1:1 dimer and prevent the initial contact between 1:1 dimer and synaptobrevin. After $\mathrm{Ca}^{2+}$ influx the protein could undergo a conformational change and loose its affinity for the 1:1 dimer or synaptobrevin. Then, synaptobrevin could rapidly bind to the 1:1 dimer, zip towards the C-terminus and fuse the membranes. As our fusion kinetics (Fig. 16) includes the replacement kinetics of the short synaptobrevin we 
cannot conclude if the binding and zippering of synaptobrevin could cause fusion in a millisecond time-scale. Alternatively, an impetus could possibly be given by the synaptotagmin - phospholipid interaction. The advantage of this model is that regulation of the initial contact between 1:1 dimer and synaptobrevin is energetically more favourable than the regulation that keeps SNAREs in a partially zippered state (configuration 2).

Configuration 2:

I showed that Tc (60-96) was less stable (Tm $\left.\sim 44^{\circ} \mathrm{C}\right)$ and Tc (1-59) was relatively more stable ( $\mathrm{Tm} \sim 66^{\circ} \mathrm{C}$ ) (Fig. 15). The thermal melt of ternary complex that contained both the synaptobrevin fragments Sb (1-59) and Sb (60-96), showed a biphasic unfolding transition (Fig.15). This suggests that two distinct domains can reside in the same helix bundle.

Here I consider N-terminally zippered trans-SNARE configuration as the molecular nature of the 'release-ready' state (Fig. 31). The hysteresis in the neuronal SNARE complex [42] is probably due to the kinetic barrier for the dissociation of synaptobrevin. In the context of a ternary complex with two synaptobrevin fragments, one could imagine that as long as one synaptobrevin fragment is bound, the other synaptobrevin fragment could freely bind and leave the complex. Indeed, I found that the first unfolding step is reversible, i.e. it did not exhibit hysteresis (data not shown). Therefore it is probable that in an $\mathrm{N}$-terminally zippered complex, the C-terminal portions could reversibly assemble. It is conceivable that in this configuration the Cterminal assembly and membrane repulsive forces could balance each other. This configuration is already suggested in other studies [49, 66, 67]. Here I provided for the first time supportive evidence that such an N-terminally zippered configuration is structurally possible. A calcium binding protein, very likely synaptotagmin 1, either alone or with the help of other factors could keep SNAREs in such an N-terminally zippered state yet at the same time it must be able to resist the strong assembly force. One could envision a factor that might be able to prevent further zippering by inserting a helix like the coiled-coil region of synaptobrevin. However, even the presence of synaptobrevin 
helix as in the $\Delta \mathrm{N}$ complex did not restrict the SNAREs from fusing membranes (Fig. 16). After the entry of $\mathrm{Ca}^{2+}$, synaptotagmin 1, with the help of calcium-dependent SNARE binding $[117,118]$ and/or phospholipid interaction [73] could give the final impetus for rapid fusion. Yet, the mechanism that would be able to keep SNAREs in such a partially zippered state is not known.

The molecular nature of the 'release-ready' state of the SNARE machinery is ambiguous. This study supports two potential configurations, through which rapid exocytosis could be accomplished. Furthermore, it revealed that the slow liposome fusion rate is largely due to 'off-pathway' reaction. In future, mutations that block various stages in the SNARE assembly in vitro should be tested in vivo to get a coherent picture about the status of SNAREs before and during exocytosis. 


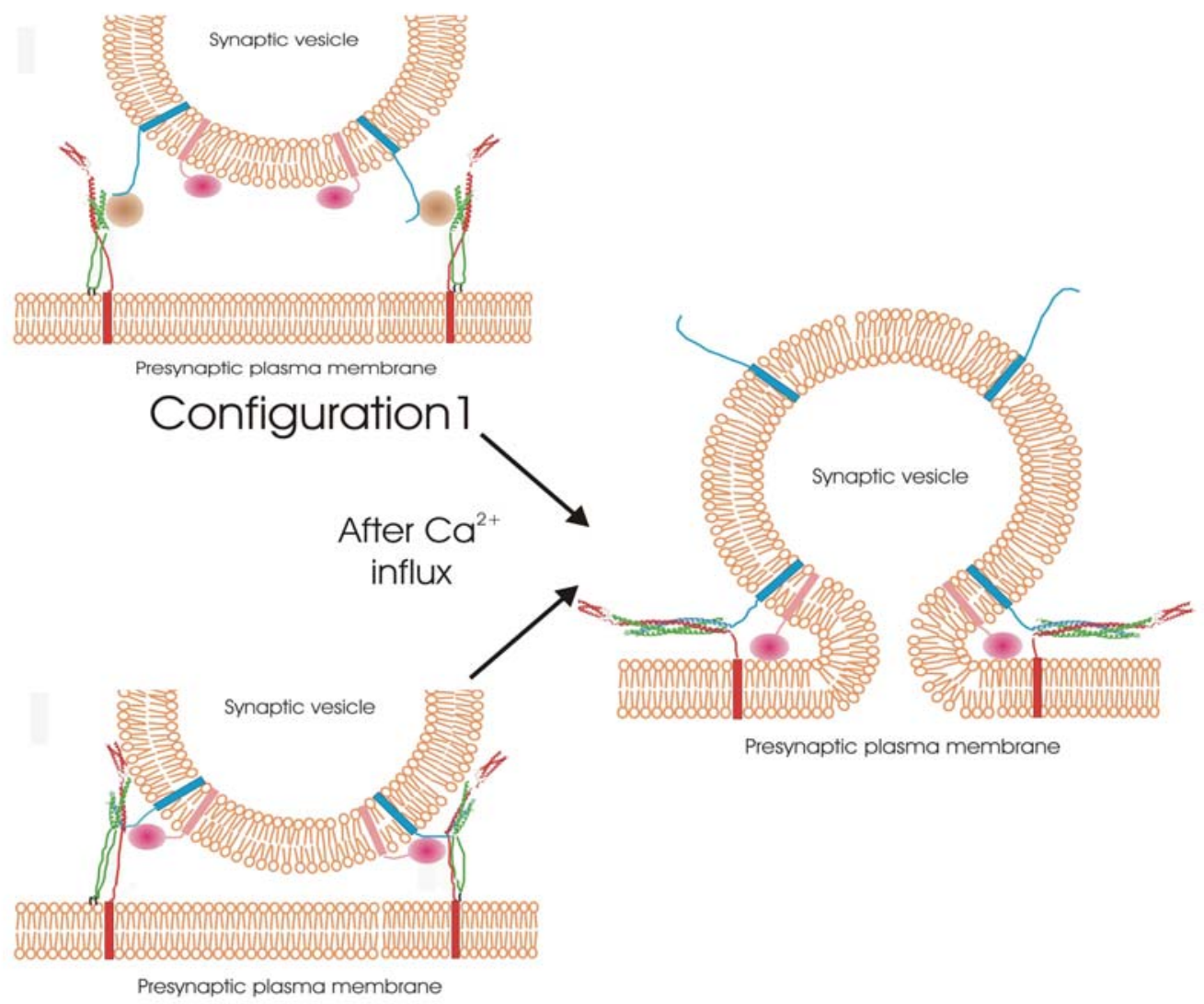

Configuration 2

\section{Figure 31 Release ready states}

In configuration 1 a 'readily-available' acceptor site is provided by 1:1 syntaxin-SNAP-25 dimer. According to this model the initial contact between 1:1 dimer and synaptobrevin is prevented by a $\mathrm{Ca}^{2+}$ binding protein (shown in brown). After $\mathrm{Ca}^{2+}$ influx this protein could undergo a structural change and lose its affinity for 1:1 dimer or synaptobrevin or both. Synaptobrevin then rapidly binds towards to the 1:1 dimer, zip towards the C-terminus and cause fusion. An impetus for fusion could also be given through $\mathrm{Ca}^{2+}$ dependent synaptotagmin-phospholipid interaction.

In configuration 2 the SNAREs are held in a partially zippered state perhaps by synaptotagmin (shown in pink). After $\mathrm{Ca}^{2+}$ influx C-terminal SNARE zippering together with $\mathrm{Ca}^{2+}$-dependent synaptotagmin-SNARE and/or phospholipid interaction could cause the final fusion. 


\subsection{Structural basis for the inhibitory role of tomosyn in exocytosis}

Neuronal exocytosis is a highly regulated event and it is primarily mediated by SNAREs. Therefore it is not surprising that several regulating proteins interact with the neuronal SNAREs. One such regulating protein is tomosyn, which is a protein of $130 \mathrm{kDa}$ with an N-terminal domain similar to that of Drosophila melanogaster tumor suppressor lethal giant larvae (Lgl) proteins, and a C-terminal R-SNARE motif. Tomosyn was isolated from the rat brain cytosol as an interacting partner of syntaxin1[39].

The SNARE motif of tomosyn interacts with the neuronal SNAREs syntaxin and SNAP-25 and forms a highly stable SNARE complex [81]. The crystal structure of the core tomosyn SNARE complex was solved in this study. It is very similar to that of the synaptobrevin SNARE complex (Fig. 18). In addition the SNARE motif of tomosyn was as efficient as synaptobrevin in forming the ternary complex (Fig. 21). The tomosyn SNARE motif in the complex cannot be replaced by synaptobrevin SNARE motif and vice versa (Fig. 19\&20). Therefore both the complexes are end products of a similar assembly reaction.

A detailed analysis of tomosyn and synaptobrevin SNARE complexes showed that both these complexes have similar hydrophobic interactions and have a comparable number of hydrogen bonds. However the intersubunit salt bridges were differentially distributed. A greater change was seen for intramolecular salt bridges, with six in the synaptobrevin helix, whereas 17 were present in tomosyn helix. This however did not alter the unfolding transition temperature of tomosyn and synaptobrevin complexes [30, 81]. Most of the changes between these two complexes were found on the surface. This in turn, prevented the interaction of complexin with the tomosyn SNARE complex (Fig. $23 \& 24)$. It is also possible that this surface change could recruit new proteins to the complex to exert some specialized functions.

Tomosyn lacks a membrane anchor and therefore the tomosyn SNARE complexes are probably ‘fusion-inactive'. However tomosyn interacts with the neuronal Q-SNAREs like the 'fusion- active' synaptobrevin. As Tomosyn competes with synaptobrevin for binding to the Q-SNAREs syntaxin and SNAP-25 it may act as a negative regulator of exocytosis. 
My data suggest that tomosyn SNARE complex does not spontaneously dissociate (Fig. 19\&20). Therefore disassembly by NSF is required to reactivate the plasma membrane Q-SNAREs to receive the fusion-active synaptobrevin.

The cAMP-dependent protein kinase (PKA) has been shown to phosphorylate tomosyn [83]. This phosphorylation prevents tomosyn from forming a SNARE complex [83]. Tomosyn has two paralogues, tomosyn 1 and tomosyn 2, and seven splice variants [80], yet this phosphorylation can occur only in the b-splice form (ubiquitously expressed) of tomosyn 1 as the other variants lack this phosphorylation site. Therefore for these isoforms/variants other regulatory mechanisms might exist. Overexpression of tomosyn causes a block in exocytosis. This block can be partially relieved by increasing $\mathrm{Ca}^{2+}$ levels [84]. Therefore the activity of tomosyn also appears to be regulated by a $\mathrm{Ca}^{2+}$ dependent mechanism.

The N-terminal region of tomosyn is homologous to the Drosophila lethal giant larvae (Lgl) proteins [77, 78]. The Lgl proteins are implicated in the establishment of apical-basal polarity. Recent studies have shown that Lgl proteins probably establish polarity by guiding vesicle fusion at specific areas of the plasma membrane [119-121]. Tomosyn is also implicated in performing a similar function. Tomosyn was suggested to guide plasmalemmal precursor vesicles to the leading edges of the neuronal growth cone by preventing the fusion of vesicles at the palm of growth cones [86].

The structure of the tomosyn SNARE complex showed that the SNARE motif of tomosyn stably interacted with the SNARE motifs of the Q-SNAREs syntaxin1 and SNAP-25. However, it is very likely that the tomosyn-Q SNARE interaction is not confined to their SNARE motifs. In tomosyn, the N-terminal region could additionally modulate Q-SNARE interaction. Furthermore, the role of tomosyn is not confined to neuronal exocytosis. The presence of tomosyn homologues throughout the eukaryotic kingdom suggests a general role for tomosyn in exocytosis. The high homology between the N-terminal domain of tomosyn and the Lgl proteins suggests that tomosyn might also be linked to an intricate signaling network like the Lgl proteins $[77,78]$. 


\subsection{Characterization of Amisyn}

Amisyn is a soluble protein of $25 \mathrm{kDa}$ and is shown to regulate the neuronal SNAREs [88, 89]. It has an N-terminal domain and a C-terminal R-SNARE motif and it resembles tomosyn in the domain architecture. The N-terminal domain, however, is similar to the C.elegans homologue of the tethering protein Uso1 [87].

It is shown that the SNARE motif of amisyn forms a stable ternary complex with the neuronal Q-SNAREs, syntaxin and SNAP-25 [88, 89]. However there is no information as to whether full-length amisyn forms a ternary complex. In this study I showed that the full-length amisyn formed a stable ternary complex (Fig. 27B) and additionally I was also able to confirm that a ternary complex is also formed by the SNARE motif of amisyn (Fig. 27A). Our intention was to crystallize the ternary complexes containing full-length and the SNARE motif of amisyn in order to get functional insights from the structure. However, I did not succeed in crystallizing the complex.

Preliminary data suggested that the full-length amisyn was as effective as the SNARE motif of amisyn in forming the SNARE complex. The N-terminal domain of certain SNAREs negatively regulates assembly [31, 122]. The N-terminal domain of amisyn does not appear to have such a role.

Sub-cellular fractionation of the neuronal tissue indicated that amisyn was largely absent in soluble fractions (Fig. 30). This was surprising as amisyn is a soluble protein and does not have a membrane anchor. It suggests that amisyn might contain unrecognized lipidation motifs or be attached to the cytoskeleton. Alternatively, amisyn might be indirectly attached to the membrane with the help of its interaction with the membrane protein syntaxin1. Furthermore, amisyn was enriched in the neuronal plasma membrane fraction and it was not detected on purified synaptic vesicles (Fig. 30) suggesting that amisyn would exert its function from the pre-synaptic plasma membrane.

The R-SNARE motif of amisyn interacts with the neuronal Q-SNAREs syntaxin and SNAP-25, as effectively as synaptobrevin. Therefore one could envision that amisyn, 
like tomosyn, could act as a negative regulator of exocytosis by competing with the 'fusion-active' synaptobrevin. Indeed, amisyn has been shown to negatively regulate exocytosis [89] but surprisingly the effect even occured without amisyn binding to syntaxin.

Lot of research is necessary in order to better understand the role of amisyn especially the function of the uncharacterized $\mathrm{N}$-terminal domain needs to be unraveled. I have established tools like purified proteins and antibodies, which could be used in future to further characterize amisyn. It would be informative to investigate the sub-cellular localization of amisyn by immunofluorescence in order to better evaluate its function. Additionally, in collaboration with Dr. Jakob Soerensen we have initiated further electrophysiological studies where we planned to infuse the intact protein, the R-SNARE motif and the N-terminal domain of amisyn in chromaffin cells and monitor their effects on exocytosis. It is my hope that these studies will shed more light on the regulatory role of amisyn. 
Summary and conclusions

\section{Summary and conclusions}

This work centered on the SNARE proteins syntaxin 1, SNAP-25, and synaptobrevin 2, which drive the discharge of neurotransmitters from synaptic vesicles. A relatively simple scenario for the role of SNAREs in neurotransmitter release is given by the zipper model, which assumes that the SNARE assembly proceeds from $\mathrm{N} \rightarrow \mathrm{C}$ terminus and the energy released during the assembly is probably sufficient to cause fusion. However, the evidence for the zipper model is largely circumstantial.

Physiologically, neurotransmitter release takes place in milliseconds, but when neuronal SNAREs are reconstituted into liposomes, fusion occurs very slowly (> several minutes). The initial part of this work addressed this long-standing controversy in the SNARE field. Biochemical analysis demonstrates the sequential assembly of neuronal SNAREs. The assembly initiates with the formation of transient dimer of syntaxin and SNAP-25, which constitutes the appropriate binding site for synaptobrevin. However, in solution this binding site is also readily occupied by a second syntaxin molecule, resulting in an 'off-pathway' 2:1 syntaxin-SNAP-25 complex. In the beginning of the work we hypothesized that the liposome fusion is probably slow because of this 'offpathway' reaction. In this study a ternary SNARE complex containing syntaxin, SNAP25 and C-terminal portion of synaptobrevin prevented this 'off-pathway' reaction. This $\Delta \mathrm{N}$ complex was also a structural mimic of syntaxin-SNAP-25 dimer. When this complex was reconstituted into liposomes the fusion was greatly enhanced. Therefore by providing an appropriate binding site and by preventing the 'off-pathway' reaction, SNAREs can rapidly fuse membranes. My data also demonstrates that the synaptobrevin binding site is restricted to the N-terminal portion of the Q-SNAREs. Thus, my study supports the zipper model.

The other part of the work focused on the soluble SNARE regulatory proteins tomosyn and amisyn. The initial detailed characterization of the SNARE motif of tomosyn led to the elucidation of the crystal structure of the core tomosyn SNARE complex, which is a four-helix bundle similar to that of the SNARE complex containing synaptobrevin. The tomosyn in the SNARE complex cannot be replaced by 
synaptobrevin and my work supports the view that tomosyn acts as a negative regulator of exocytosis. The full-length and the SNARE motif of amisyn, like tomosyn, formed a stable ternary complex with syntaxin and SNAP-25. Preliminary experiments suggested that amisyn also acts as a negative regulator of exocytosis. 


\section{Bibliography}

1. Mellman, I. and G. Warren, The road taken: past and future foundations of membrane traffic. Cell, 2000. 100(1): p. 99-112.

2. Jahn, R., Principles of exocytosis and membrane fusion. Ann N Y Acad Sci, 2004. 1014: p. 170-8.

3. Jahn, R., T. Lang, and T.C. Sudhof, Membrane fusion. Cell, 2003. 112(4): p. 51933.

4. Ungermann, C. and D. Langosch, Functions of SNAREs in intracellular membrane fusion and lipid bilayer mixing. J Cell Sci, 2005. 118(Pt 17): p. 381928.

5. Chen, Y.A. and R.H. Scheller, SNARE-mediated membrane fusion. Nat Rev Mol Cell Biol, 2001. 2(2): p. 98-106.

6. Bonifacino, J.S. and B.S. Glick, The mechanisms of vesicle budding and fusion. Cell, 2004. 116(2): p. 153-66.

7. Hermann, G.J., et al., Mitochondrial fusion in yeast requires the transmembrane GTPase Fzo1p. J Cell Biol, 1998. 143(2): p. 359-73.

8. Sesaki, H. and R.E. Jensen, UGO1 encodes an outer membrane protein required for mitochondrial fusion. J Cell Biol, 2001. 152(6): p. 1123-34.

9. Lerman, J.C., et al., Structural analysis of the neuronal SNARE protein syntaxin1A. Biochemistry, 2000. 39(29): p. 8470-9.

10. Fernandez, I., et al., Three-dimensional structure of an evolutionarily conserved N-terminal domain of syntaxin 1A. Cell, 1998. 94(6): p. 841-9.

11. Misura, K.M., et al., Three-dimensional structure of the amino-terminal domain of syntaxin 6, a SNAP-25 C homolog. Proc Natl Acad Sci U S A, 2002. 99(14): p. 9184-9.

12. Dulubova, I., et al., Vam3p structure reveals conserved and divergent properties of syntaxins. Nat Struct Biol, 2001. 8(3): p. 258-64.

13. Gonzalez, L.C., Jr., W.I. Weis, and R.H. Scheller, A novel snare N-terminal domain revealed by the crystal structure of Sec22b. J Biol Chem, 2001. 276(26): p. 24203-11. 
14. Tochio, H., et al., An autoinhibitory mechanism for nonsyntaxin SNARE proteins revealed by the structure of Ykt6p. Science, 2001. 293(5530): p. 698-702.

15. Lu, J., et al., Solution structure of the Vam7p PX domain. Biochemistry, 2002. 41(19): p. 5956-62.

16. Sollner, T., et al., SNAP receptors implicated in vesicle targeting and fusion. Nature, 1993. 362(6418): p. 318-24.

17. Rothman, J.E., Mechanisms of intracellular protein transport. Nature, 1994. 372(6501): p. 55-63.

18. Bennett, M.K., N. Calakos, and R.H. Scheller, Syntaxin: a synaptic protein implicated in docking of synaptic vesicles at presynaptic active zones. Science, 1992. 257(5067): p. 255-9.

19. Aguado, F., et al., Syntaxin $1 \mathrm{~A}$ and $1 \mathrm{~B}$ display distinct distribution patterns in the rat peripheral nervous system. Neuroscience, 1999. 88(2): p. 437-46.

20. Ruiz-Montasell, B., et al., Differential distribution of syntaxin isoforms 1A and 1B in the rat central nervous system. Eur J Neurosci, 1996. 8(12): p. 2544-52.

21. Oyler, G.A., et al., The identification of a novel synaptosomal-associated protein, SNAP-25, differentially expressed by neuronal subpopulations. J Cell Biol, 1989. 109(6 Pt 1): p. 3039-52.

22. Hess, D.T., et al., The $25 \mathrm{kDa}$ synaptosomal-associated protein SNAP-25 is the major methionine-rich polypeptide in rapid axonal transport and a major substrate for palmitoylation in adult CNS. J Neurosci, 1992. 12(12): p. 4634-41.

23. Bark, I.C., et al., Differential expression of SNAP-25 protein isoforms during divergent vesicle fusion events of neural development. Proc Natl Acad Sci U S A, 1995. 92(5): p. 1510-4.

24. Baumert, M., et al., Synaptobrevin: an integral membrane protein of 18,000 daltons present in small synaptic vesicles of rat brain. Embo J, 1989. 8(2): p. 37984.

25. Sutton, R.B., et al., Crystal structure of a SNARE complex involved in synaptic exocytosis at 2.4 A resolution. Nature, 1998. 395(6700): p. 347-53. 
26. Fasshauer, D., et al., Conserved structural features of the synaptic fusion complex: SNARE proteins reclassified as Q- and R-SNAREs. Proc Natl Acad Sci U S A, 1998. 95(26): p. 15781-6.

27. Bock, J.B., et al., A genomic perspective on membrane compartment organization. Nature, 2001. 409(6822): p. 839-41.

28. Hanson, P.I., J.E. Heuser, and R. Jahn, Neurotransmitter release - four years of SNARE complexes. Curr Opin Neurobiol, 1997. 7(3): p. 310-5.

29. Hay, J.C. and R.H. Scheller, SNAREs and NSF in targeted membrane fusion. Curr Opin Cell Biol, 1997. 9(4): p. 505-12.

30. Fasshauer, D., et al., Structural changes are associated with soluble Nethylmaleimide-sensitive fusion protein attachment protein receptor complex formation. J Biol Chem, 1997. 272(44): p. 28036-41.

31. Margittai, M., et al., Single-molecule fluorescence resonance energy transfer reveals a dynamic equilibrium between closed and open conformations of syntaxin 1. Proc Natl Acad Sci U S A, 2003. 100(26): p. 15516-21.

32. Parlati, F., et al., Rapid and efficient fusion of phospholipid vesicles by the alphahelical core of a SNARE complex in the absence of an N-terminal regulatory domain. Proc Natl Acad Sci U S A, 1999. 96(22): p. 12565-70.

33. Brenner, S., The genetics of Caenorhabditis elegans. Genetics, 1974. 77(1): p. 7194.

34. Hata, Y., C.A. Slaughter, and T.C. Sudhof, Synaptic vesicle fusion complex contains unc-18 homologue bound to syntaxin. Nature, 1993. 366(6453): p. 34751.

35. Garcia, E.P., et al., A rat brain Sec1 homologue related to Rop and UNC18 interacts with syntaxin. Proc Natl Acad Sci U S A, 1994. 91(6): p. 2003-7.

36. Misura, K.M., R.H. Scheller, and W.I. Weis, Three-dimensional structure of the neuronal-Sec1-syntaxin 1a complex. Nature, 2000. 404(6776): p. 355-62.

37. Pevsner, J., et al., Specificity and regulation of a synaptic vesicle docking complex. Neuron, 1994. 13(2): p. 353-61. 
38. Verhage, M., et al., Synaptic assembly of the brain in the absence of neurotransmitter secretion. Science, 2000. 287(5454): p. 864-9.

39. Fujita, Y., et al., Tomosyn: a syntaxin-1-binding protein that forms a novel complex in the neurotransmitter release process. Neuron, 1998. 20(5): p. 905-15.

40. Richmond, J.E., R.M. Weimer, and E.M. Jorgensen, An open form of syntaxin bypasses the requirement for UNC-13 in vesicle priming. Nature, 2001. 412(6844): p. 338-41.

41. Sassa, T., et al., Regulation of the UNC-18-Caenorhabditis elegans syntaxin complex by UNC-13. J Neurosci, 1999. 19(12): p. 4772-7.

42. Fasshauer, D., et al., SNARE assembly and disassembly exhibit a pronounced hysteresis. Nat Struct Biol, 2002. 9(2): p. 144-51.

43. Barrick, D. and F.M. Hughson, Irreversible assembly of membrane fusion machines. Nat Struct Biol, 2002. 9(2): p. 78-80.

44. Mayer, A., W. Wickner, and A. Haas, Sec18p (NSF)-driven release of Sec17p (alpha-SNAP) can precede docking and fusion of yeast vacuoles. Cell, 1996. 85(1): p. 83-94.

45. Fasshauer, D. and M. Margittai, A transient N-terminal interaction of SNAP-25 and syntaxin nucleates SNARE assembly. J Biol Chem, 2004. 279(9): p. 7613-21.

46. Fiebig, K.M., et al., Folding intermediates of SNARE complex assembly. Nat Struct Biol, 1999. 6(2): p. 117-23.

47. Antonin, W., et al., Crystal structure of the endosomal SNARE complex reveals common structural principles of all SNAREs. Nat Struct Biol, 2002. 9(2): p. 10711.

48. Chen, X., et al., Three-dimensional structure of the complexin/SNARE complex. Neuron, 2002. 33(3): p. 397-409.

49. $\mathrm{Xu}, \mathrm{T}$., et al., Inhibition of SNARE complex assembly differentially affects kinetic components of exocytosis. Cell, 1999. 99(7): p. 713-22.

50. Broadie, K., et al., Syntaxin and synaptobrevin function downstream of vesicle docking in Drosophila. Neuron, 1995. 15(3): p. 663-73. 
51. Washbourne, P., et al., Genetic ablation of the t-SNARE SNAP-25 distinguishes mechanisms of neuroexocytosis. Nat Neurosci, 2002. 5(1): p. 19-26.

52. Schoch, S., et al., SNARE function analyzed in synaptobrevin/VAMP knockout mice. Science, 2001. 294(5544): p. 1117-22.

53. Littleton, J.T., et al., Temperature-sensitive paralytic mutations demonstrate that synaptic exocytosis requires SNARE complex assembly and disassembly. Neuron, 1998. 21(2): p. 401-13.

54. Wei, S., et al., Exocytotic mechanism studied by truncated and zero layer mutants of the C-terminus of SNAP-25. Embo J, 2000. 19(6): p. 1279-89.

55. Fergestad, T., et al., Targeted mutations in the syntaxin H3 domain specifically disrupt SNARE complex function in synaptic transmission. J Neurosci, 2001. 21(23): p. 9142-50.

56. Blasi, J., et al., Botulinum neurotoxin A selectively cleaves the synaptic protein SNAP-25. Nature, 1993. 365(6442): p. 160-3.

57. Sweeney, S.T., et al., Targeted expression of tetanus toxin light chain in Drosophila specifically eliminates synaptic transmission and causes behavioral defects. Neuron, 1995. 14(2): p. 341-51.

58. Capogna, M., et al., Ca2+ or Sr2+ partially rescues synaptic transmission in hippocampal cultures treated with botulinum toxin A and C, but not tetanus toxin. J Neurosci, 1997. 17(19): p. 7190-202.

59. Schiavo, G., et al., Tetanus and botulinum-B neurotoxins block neurotransmitter release by proteolytic cleavage of synaptobrevin. Nature, 1992. 359(6398): p. 832-5.

60. Yamasaki, S., et al., Cleavage of members of the synaptobrevin/VAMP family by types D and F botulinal neurotoxins and tetanus toxin. J Biol Chem, 1994. 269(17): p. 12764-72.

61. $\mathrm{Xu}, \mathrm{T}$., et al., Early requirement for alpha-SNAP and NSF in the secretory cascade in chromaffin cells. Embo J, 1999. 18(12): p. 3293-304. 
62. Nagy, G., et al., Protein kinase C-dependent phosphorylation of synaptosomeassociated protein of $25 \mathrm{kDa}$ at Ser187 potentiates vesicle recruitment. J Neurosci, 2002. 22(21): p. 9278-86.

63. Nagy, G., et al., Regulation of releasable vesicle pool sizes by protein kinase Adependent phosphorylation of SNAP-25. Neuron, 2004. 41(3): p. 417-29.

64. Sorensen, J.B., et al., Differential control of the releasable vesicle pools by SNAP-25 splice variants and SNAP-23. Cell, 2003. 114(1): p. 75-86.

65. Weber, T., et al., SNAREpins: minimal machinery for membrane fusion. Cell, 1998. 92(6): p. 759-72.

66. Hua, S.Y. and M.P. Charlton, Activity-dependent changes in partial VAMP complexes during neurotransmitter release. Nat Neurosci, 1999. 2(12): p. 107883.

67. Chen, Y.A., S.J. Scales, and R.H. Scheller, Sequential SNARE assembly underlies priming and triggering of exocytosis. Neuron, 2001. 30(1): p. 161-70.

68. Hayashi, T., et al., Synaptic vesicle membrane fusion complex: action of clostridial neurotoxins on assembly. Embo J, 1994. 13(21): p. 5051-61.

69. Sudhof, T.C., The synaptic vesicle cycle. Annu Rev Neurosci, 2004. 27: p. 50947.

70. Koh, T.W. and H.J. Bellen, Synaptotagmin I, a Ca2+ sensor for neurotransmitter release. Trends Neurosci, 2003. 26(8): p. 413-22.

71. Davis, A.F., et al., Kinetics of synaptotagmin responses to $\mathrm{Ca} 2+$ and assembly with the core SNARE complex onto membranes. Neuron, 1999. 24(2): p. 363-76.

72. Li, C., B.A. Davletov, and T.C. Sudhof, Distinct $\mathrm{Ca} 2+$ and Sr2+ binding properties of synaptotagmins. Definition of candidate $\mathrm{Ca} 2+$ sensors for the fast and slow components of neurotransmitter release. J Biol Chem, 1995. 270(42): p. 24898-902.

73. Shin, O.H., et al., Sr2+ binding to the Ca2+ binding site of the synaptotagmin 1 C2B domain triggers fast exocytosis without stimulating SNARE interactions. Neuron, 2003. 37(1): p. 99-108. 
74. Mechler, B.M., W. McGinnis, and W.J. Gehring, Molecular cloning of lethal(2)giant larvae, a recessive oncogene of Drosophila melanogaster. Embo J, 1985. 4(6): p. 1551-7.

75. Kim, Y.S., et al., The rgl-1 is a legitimate homologue of lethal giant larvae recessive oncogene in rat. Int J Oncol, 2002. 20(6): p. 1219-25.

76. Lehman, K., et al., Yeast homologues of tomosyn and lethal giant larvae function in exocytosis and are associated with the plasma membrane SNARE, Sec9. J Cell Biol, 1999. 146(1): p. 125-40.

77. Justice, N.J. and Y.N. Jan, A lethal giant kinase in cell polarity. Nat Cell Biol, 2003. 5(4): p. 273-4.

78. Humbert, P., S. Russell, and H. Richardson, Dlg, Scribble and Lgl in cell polarity, cell proliferation and cancer. Bioessays, 2003. 25(6): p. 542-53.

79. Yokoyama, S., et al., Three splicing variants of tomosyn and identification of their syntaxin-binding region. Biochem Biophys Res Commun, 1999. 256(1): p. 218-22.

80. Groffen, A.J., et al., Two distinct genes drive expression of seven tomosyn isoforms in the mammalian brain, sharing a conserved structure with a unique variable domain. J Neurochem, 2005. 92(3): p. 554-68.

81. Hatsuzawa, K., et al., The R-SNARE motif of tomosyn forms SNARE core complexes with syntaxin 1 and SNAP-25 and down-regulates exocytosis. J Biol Chem, 2003. 278(33): p. 31159-66.

82. Pobbati, A.V., et al., Structural basis for the inhibitory role of tomosyn in exocytosis. J Biol Chem, 2004. 279(45): p. 47192-200.

83. Baba, T., et al., PKA-catalyzed phosphorylation of tomosyn and its implication in Ca2+-dependent exocytosis of neurotransmitter. J Cell Biol, 2005. 170(7): p. 1113-25.

84. Yizhar, O., et al., Tomosyn inhibits priming of large dense-core vesicles in a calcium-dependent manner. Proc Natl Acad Sci U S A, 2004. 101(8): p. 2578-83. 
85. Widberg, C.H., et al., Tomosyn interacts with the t-SNAREs syntaxin4 and SNAP23 and plays a role in insulin-stimulated GLUT4 translocation. J Biol Chem, 2003. 278(37): p. 35093-101.

86. Sakisaka, T., et al., Regulation of SNAREs by tomosyn and ROCK: implication in extension and retraction of neurites. J Cell Biol, 2004. 166(1): p. 17-25.

87. Nakajima, H., et al., A cytoskeleton-related gene, uso1, is required for intracellular protein transport in Saccharomyces cerevisiae. J Cell Biol, 1991. 113(2): p. 245-60.

88. Scales, S.J., et al., Amisyn, a novel syntaxin-binding protein that may regulate SNARE complex assembly. J Biol Chem, 2002. 277(31): p. 28271-9.

89. Constable, J.R., et al., Amisyn regulates exocytosis and fusion pore stability by both syntaxin-dependent and syntaxin-independent mechanisms. J Biol Chem, 2005. 280(36): p. 31615-23.

90. Fasshauer, D., et al., Identification of a minimal core of the synaptic SNARE complex sufficient for reversible assembly and disassembly. Biochemistry, 1998. 37(29): p. 10354-62.

91. Margittai, M., H. Otto, and R. Jahn, A stable interaction between syntaxin 1a and synaptobrevin 2 mediated by their transmembrane domains. FEBS Lett, 1999. 446(1): p. 40-4.

92. Margittai, M., et al., Homo- and heterooligomeric SNARE complexes studied by site-directed spin labeling. J Biol Chem, 2001. 276(16): p. 13169-77.

93. Pabst, S., et al., Rapid and selective binding to the synaptic SNARE complex suggests a modulatory role of complexins in neuroexocytosis. J Biol Chem, 2002. 277(10): p. 7838-48.

94. Fasshauer, D., et al., Mixed and non-cognate SNARE complexes. Characterization of assembly and biophysical properties. J Biol Chem, 1999. 274(22): p. 15440-6.

95. Inoue, H., H. Nojima, and H. Okayama, High efficiency transformation of Escherichia coli with plasmids. Gene, 1990. 96(1): p. 23-8. 
96. Bradford, M.M., A rapid and sensitive method for the quantitation of microgram quantities of protein utilizing the principle of protein-dye binding. Anal Biochem, 1976. 72: p. 248-54.

97. Laemmli, U.K., Cleavage of structural proteins during the assembly of the head of bacteriophage T4. Nature, 1970. 227(5259): p. 680-5.

98. Schagger, H. and G. von Jagow, Tricine-sodium dodecyl sulfate-polyacrylamide gel electrophoresis for the separation of proteins in the range from 1 to $100 \mathrm{kDa}$. Anal Biochem, 1987. 166(2): p. 368-79.

99. Otwinowski, Z., and Minor, W. Methods Enzymol. , (1997). 276: p. 307-326.

100. Winn, M.D., et al., Ongoing developments in CCP4 for high-throughput structure determination. Acta Crystallogr D Biol Crystallogr, 2002. 58(Pt 11): p. 1929-36.

101. Potterton, E., et al., A graphical user interface to the CCP4 program suite. Acta Crystallogr D Biol Crystallogr, 2003. 59(Pt 7): p. 1131-7.

102. Navaza, J., Implementation of molecular replacement in AMoRe. Acta Crystallogr D Biol Crystallogr, 2001. 57(Pt 10): p. 1367-72.

103. Ernst, J.A. and A.T. Brunger, High resolution structure, stability, and synaptotagmin binding of a truncated neuronal SNARE complex. J Biol Chem, 2003. 278(10): p. 8630-6.

104. Perrakis, A., R. Morris, and V.S. Lamzin, Automated protein model building combined with iterative structure refinement. Nat Struct Biol, 1999. 6(5): p. 45863.

105. McRee, D.E., XtalView/Xfit--A versatile program for manipulating atomic coordinates and electron density. J Struct Biol, 1999. 125(2-3): p. 156-65.

106. Murshudov, G.N., et al., Efficient anisotropic refinement of macromolecular structures using FFT. Acta Crystallogr D Biol Crystallogr, 1999. 55 ( Pt 1): p. 247-55.

107. Thompson, J.D., D.G. Higgins, and T.J. Gibson, CLUSTAL W: improving the sensitivity of progressive multiple sequence alignment through sequence weighting, position-specific gap penalties and weight matrix choice. Nucleic Acids Res, 1994. 22(22): p. 4673-80. 
108. Jahn, R., et al., A 38,000-dalton membrane protein (p38) present in synaptic vesicles. Proc Natl Acad Sci U S A, 1985. 82(12): p. 4137-41.

109. Huttner, W.B., et al., Synapsin I (protein I), a nerve terminal-specific phosphoprotein. III. Its association with synaptic vesicles studied in a highly purified synaptic vesicle preparation. J Cell Biol, 1983. 96(5): p. 1374-88.

110. Nagy, A., et al., The preparation and characterization of synaptic vesicles of high purity. Brain Res, 1976. 109(2): p. 285-309.

111. Pabst, S., et al., Selective interaction of complexin with the neuronal SNARE complex. Determination of the binding regions. J Biol Chem, 2000. 275(26): p. 19808-18.

112. Bracher, A., et al., X-ray structure of a neuronal complexin-SNARE complex from squid. J Biol Chem, 2002. 277(29): p. 26517-23.

113. Sakaba, T., et al., Distinct kinetic changes in neurotransmitter release after SNARE protein cleavage. Science, 2005. 309(5733): p. 491-4.

114. Hu, K., et al., Vesicular restriction of synaptobrevin suggests a role for calcium in membrane fusion. Nature, 2002. 415(6872): p. 646-50.

115. Kweon, D.H., C.S. Kim, and Y.K. Shin, Regulation of neuronal SNARE assembly by the membrane. Nat Struct Biol, 2003. 10(6): p. 440-7.

116. de Haro, L., et al., Ca2+/calmodulin transfers the membrane-proximal lipidbinding domain of the v-SNARE synaptobrevin from cis to trans bilayers. Proc Natl Acad Sci U S A, 2004. 101(6): p. 1578-83.

117. Chapman, E.R., et al., Ca2+ regulates the interaction between synaptotagmin and syntaxin 1. J Biol Chem, 1995. 270(40): p. 23667-71.

118. Li, C., et al., $\mathrm{Ca}(2+)$-dependent and -independent activities of neural and nonneural synaptotagmins. Nature, 1995. 375(6532): p. 594-9.

119. Bilder, D., M. Li, and N. Perrimon, Cooperative regulation of cell polarity and growth by Drosophila tumor suppressors. Science, 2000. 289(5476): p. 113-6.

120. Musch, A., et al., Mammalian homolog of Drosophila tumor suppressor lethal (2) giant larvae interacts with basolateral exocytic machinery in Madin-Darby canine kidney cells. Mol Biol Cell, 2002. 13(1): p. 158-68. 
121. Zhang, X., et al., Lethal giant larvae proteins interact with the exocyst complex and are involved in polarized exocytosis. J Cell Biol, 2005. 170(2): p. 273-83.

122. Nicholson, K.L., et al., Regulation of SNARE complex assembly by an Nterminal domain of the t-SNARE Sso1p. Nat Struct Biol, 1998. 5(9): p. 793-802. 


\section{List of publications}

1. Pobbati, A.V., Razeto, A., Böddener, M., Becker, S., Fasshauer, D., Structural basis for the inhibitory role of tomosyn in exocytosis. J Biol Chem, 2004. 279(45): p. 47192-200.

2. Pobbati, A.V., Stein, A., Fasshauer, D., SNAREs catalyse membrane fusion. (manuscript in preparation). 


\section{Acknowledgements}

I am indebted to Dr. Dirk Fasshauer for the supervision, guidance and for teaching me how to do science.

I thank Prof. Reinhard Jahn for the guidance support and also for providing a very nice working environment. I thank my Ph.D. committee members, Prof. Wolfgang Liebl and Prof. Ralf Ficner for their time and guidance.

It was wonderful being a part of 'Team Future' and I thank all the members for the care and support. I thank all my lab members, colleagues and friends for providing a nice and stimulating atmosphere.

I thank Dr. Steffen Burkhardt and the coordination office for making my life easier from the beginning of my stay in Germany.

I am grateful to my family, especially my mother for all the sacrifices made for me. 


\section{Appendix}

Crystallographic data

\section{Summary of data collection and refinement statistics}

\section{Data collection}

Space group

Unit cell dimensions

Resolution range/outer shell ( $\AA$ )

Completeness overall/outer shell (\%)

Redundancy overall/outer shell

$R_{\text {merge }}$ overall/outer shell

Mean $I / \sigma$ overall/outer shell

Refinement

Reflections unique/free

$R_{\text {factor }} / R_{\text {free }}(\%)$

No. residues/water molecules

Ramachandran plot (\%)

Most favored

Additionally allowed

Generously allowed

Disallowed

r.m.s.d. bond distance $(\AA ̊)$

r.m.s.d. bond angle $\left(^{\circ}\right)$

Protein average $B$-factor $\left(\AA^{2}\right)$ $(\mathrm{A} / \mathrm{B} / \mathrm{C} / \mathrm{D})$

Water average $B$-factor $\left(\AA^{2}\right)$
$\mathrm{C} 2$

$$
\begin{gathered}
a=126.1 \AA, b=35.6 \AA, c=93.5 \AA, \alpha=90^{\circ}, \beta= \\
132.4^{\circ}, \gamma=90^{\circ}
\end{gathered}
$$

19.39-2.00/2.03-2.00

99.5/97.1

$3.5 / 3.4$

$0.051 / 0.145$

$19 / 6.4$

19,950/1077

$16.71 / 22.5$

$252 / 278$

99.6

0.0

0.4

0.0

0.014

1.2

24.3/27.8/31.3/29.8

46.6 


\section{Abbreviations}

\begin{tabular}{|c|c|}
\hline сAMP & cyclic adenosine monophosphate \\
\hline cps & counts per second \\
\hline $\mathrm{CNBr}$ & cyanogen bromide \\
\hline EDTA & ethylene diamine tetraacetic acid \\
\hline EPR & Electron paramagnetic resonance \\
\hline GdnHCl & guanidine hydrochloride \\
\hline IPTG & isopropyl-beta-D-thiogalactopyranoside \\
\hline kDa & kilo Dalton \\
\hline LB & Luria-Bertani \\
\hline MES & (N-Morpholino) ethane sulphonic acid \\
\hline MPD & 2-methyl-2, 4-pentandiol \\
\hline NBD & 7-nitro-2-1, 3-benzooxadiazol-4-yl \\
\hline NSF & N-ethyl-maleimide sensitive factor \\
\hline PAGE & polyacyrlamide gel electrophoresis \\
\hline PC12 & cell line derived from pheochromocytoma \\
\hline PMSF & phenylmethylsulphonylfluoride \\
\hline PtdIns (3) P & phosphatidylinositol-3-phosphate \\
\hline RNAi & RNA interference \\
\hline SCG & superior cervical ganglion \\
\hline SDS & sodium dodecyl sulphate \\
\hline SNAP-25 & synaptosomal associated protein of $25 \mathrm{kDa}$ \\
\hline SNARE & soluble (NSF) attachment protein receptor \\
\hline TB & terrific broth \\
\hline Тc & ternary complex \\
\hline VAMP & vesicle-associated membrane protein \\
\hline
\end{tabular}




\section{Curriculum Vitae}

\section{Personal information}

Name: $\quad$ Ajaybabu Pobbati Venkatesan

Date of birth: 18.02.1979

Place of birth: Chennai, India

Nationality: Indian

\section{Education}

2002 - Present: Doctoral training in the International Molecular Biology program, Georg-August-Universität Göttingen.

2001 - 2002: $\quad$ Coursework in the International Molecular Biology program, Georg-August-Universität Göttingen.

1996-2001:

Master's studies, Life Sciences with specialization in Biotechnology, 5-year integrated course, Bharathidasan University, India 